\title{
Ethynylhydroxycarbene $(\mathrm{H}-\mathrm{C} \equiv \mathrm{C}-\ddot{\mathrm{C}}-\mathrm{OH})$
}

Bastian Bernhardt, Marcel Ruth, André K. Eckhardt, and Peter R. Schreiner*

Institute of Organic Chemistry, Justus Liebig University, Heinrich-Buff-Ring 17, 35392 Giessen, Germany

*prs@uni-giessen.de

\section{Table of Contents}

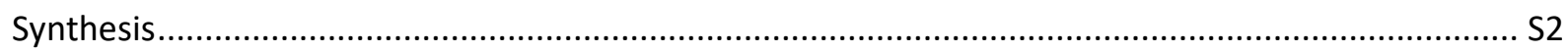

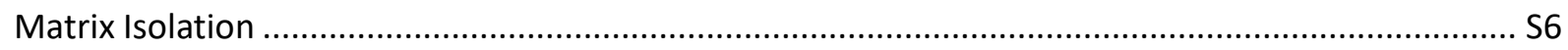

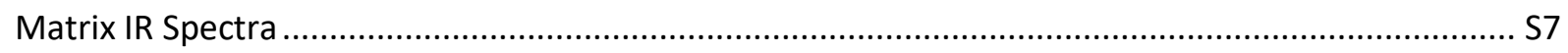

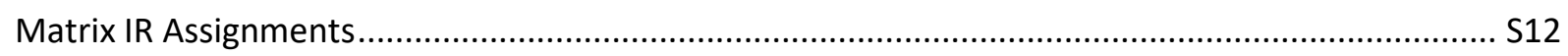

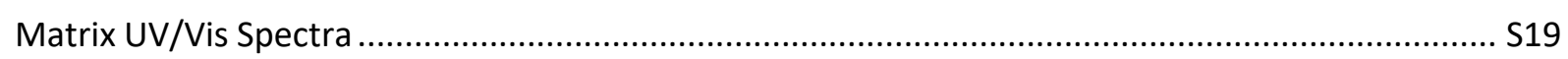

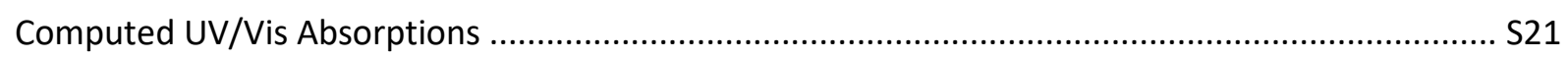

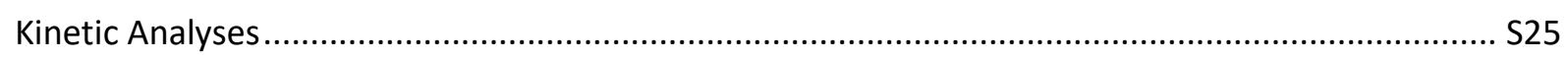

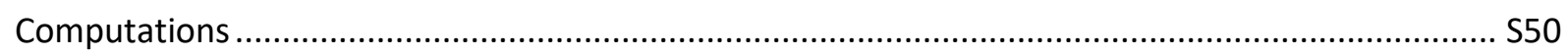

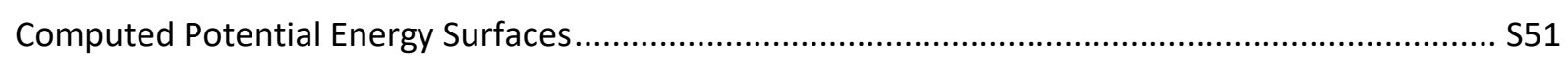

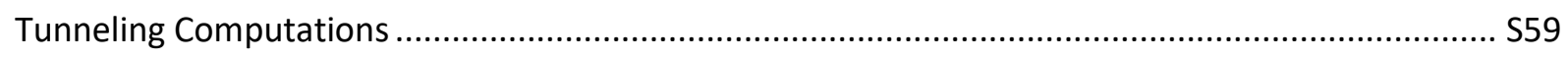

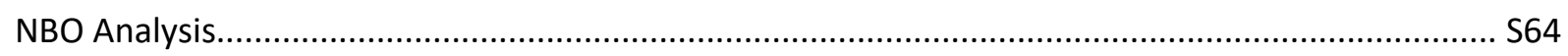

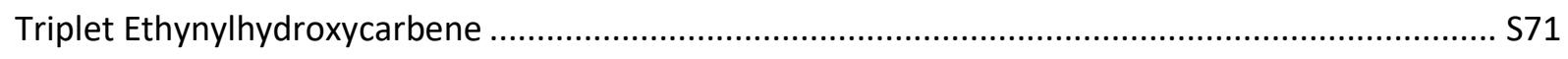

Cartesian Coordinates of Computationally Optimized Geometries.................................................. S73

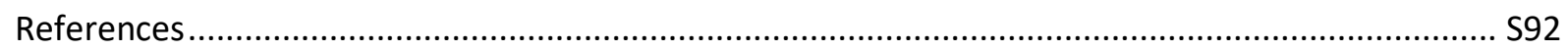

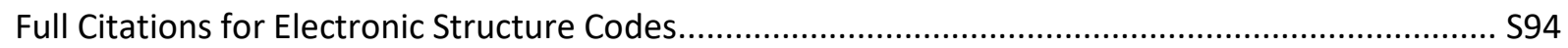




\section{Synthesis}

Ethynylglyoxylic acid ethyl ester (10)

A solution of $0.700 \mathrm{~g}(5.635 \mathrm{mmol})$ ethyl 2-hydroxybut-3-ynoate (11) in $23.4 \mathrm{~mL}$ dichloromethane (DCM) was prepared. $2.629 \mathrm{~g}(6.199 \mathrm{mmol})$ Dess-Martin periodinane (DMP) were added within $1 \mathrm{~min}$. After stirring the reaction mixture for $45 \mathrm{~min}$ the solvent was evaporated under reduced pressure. The crude product was purified by column chromatography with pentane/diethyl ether $\left(10: 1 ; R_{\mathrm{f}}=0.12\right)$. The collected product fractions were washed three times with $20 \mathrm{~mL} \mathrm{H}_{2} \mathrm{O}$. The solvent was removed under reduced pressure and $315 \mathrm{mg}$ (44\%) 10 were obtained as a yellow to brown liquid.

${ }^{1} \mathrm{H}$ NMR $\left(400 \mathrm{MHz}^{\mathrm{CDCl}} 3\right.$ ): $\delta=4.39\left(\mathrm{q},{ }^{3} \mathrm{~J}=7.2 \mathrm{~Hz}, 2 \mathrm{H}\right), 3.60(\mathrm{~s}, 1 \mathrm{H}), 1.40\left(\mathrm{t},{ }^{3} \mathrm{~J}=7.2 \mathrm{~Hz}, 3 \mathrm{H}\right) \mathrm{ppm}$.

${ }^{13} \mathrm{C}\left\{{ }^{1} \mathrm{H}\right\} \mathrm{NMR}\left(101 \mathrm{MHz}, \mathrm{CDCl}_{3}\right): \delta=169.4,158.7,85.2,79.9,63.7,14.1 \mathrm{ppm}$.

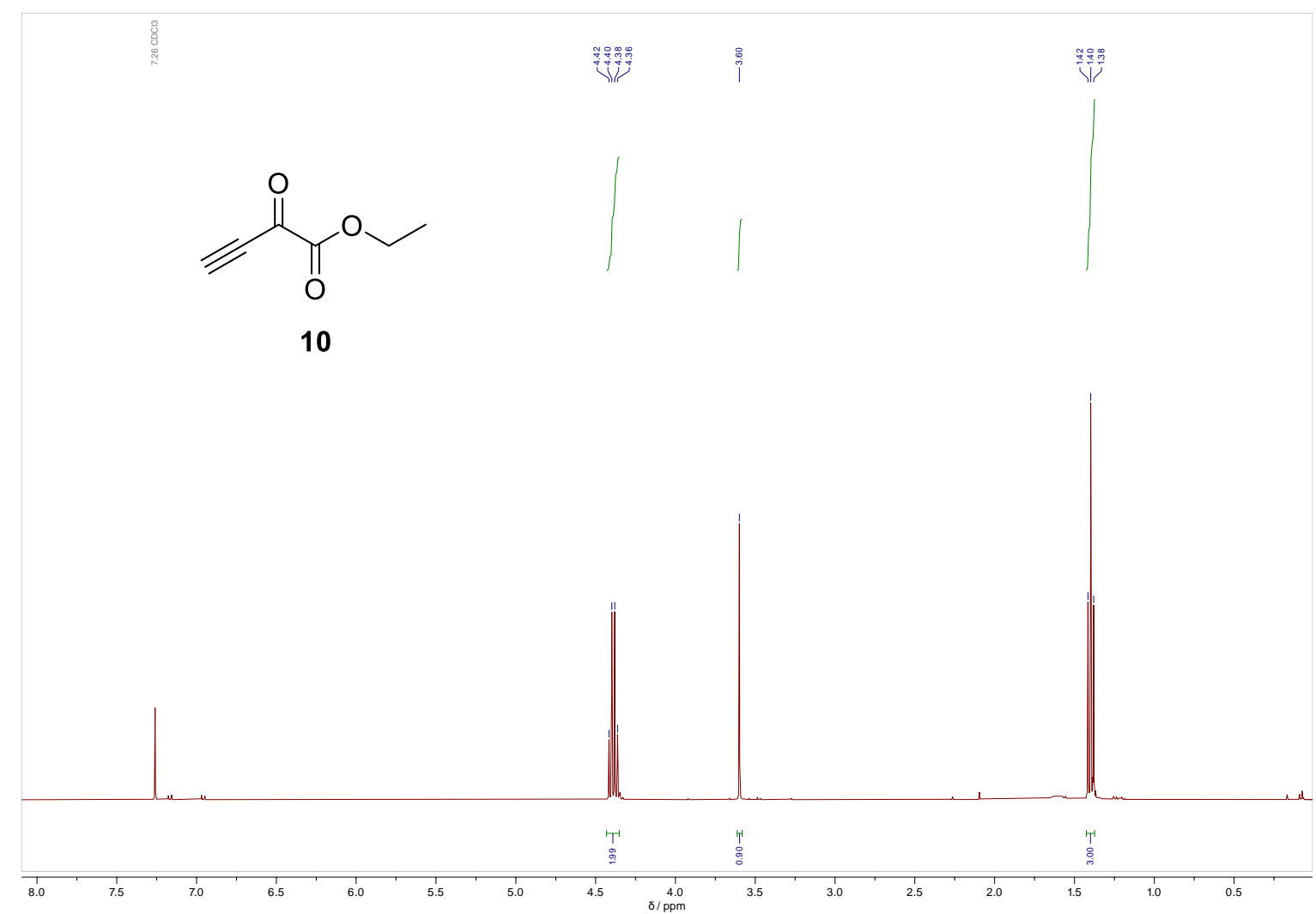

Figure S1: ${ }^{1} \mathrm{H}$ NMR spectrum of ethynylglyoxylic acid ethyl ester (10). 


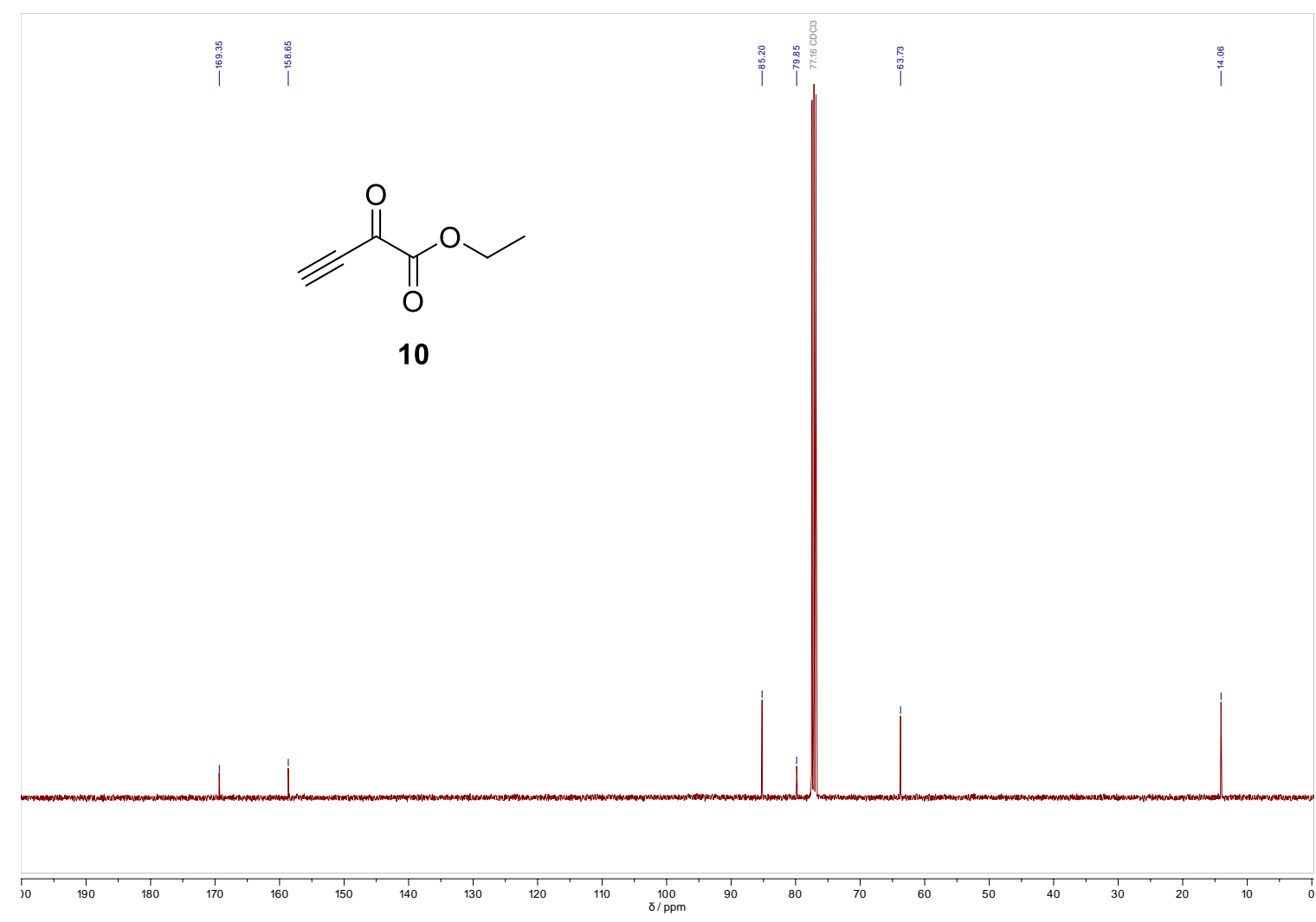

Figure S2: ${ }^{13} \mathrm{C}\left\{{ }^{1} \mathrm{H}\right\}$ NMR spectrum of ethynylglyoxylic acid ethyl ester (10).

Unsuccessful attempts to synthesize ethynylglyoxylic acid ethyl ester (10)

Our first idea for the synthesis of $\mathbf{1 0}$ or its free acid (26) was generation of trimethylsilylethynylglyoxylic acid ethyl ester (24) and subsequent cleavage of the TMS group as depicted in Scheme S1. However, only the first reaction step shown was successful. Attempts to yield $\mathbf{2 6}$ via saponification of $\mathbf{1 0}$ obtained from above described protocol failed as did saponification of $\mathbf{2 4}$. In both cases the reactions occurred with full conversion of the starting material, but complex mixtures of unidentified decomposition products resulted. Presumably, the free acids $\mathbf{4}$ and $\mathbf{2 5}$ are not stable under the applied reaction conditions.

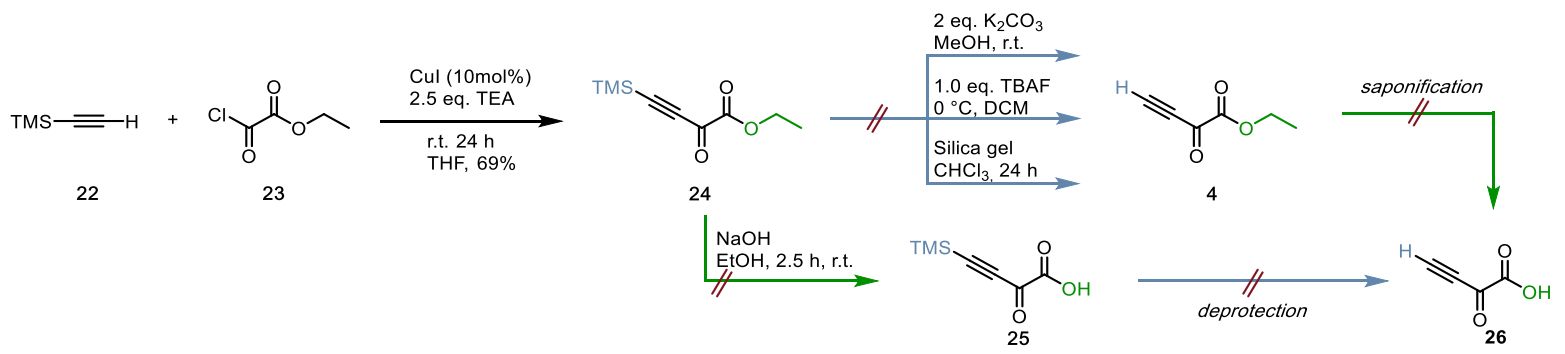

Scheme S1: Unsuccessful attempts to synthesize ethynylglyoxylic acid ethyl ester (10) and its free acid (26). 
To a solution of $412.5 \mathrm{mg}$ ( $2.166 \mathrm{mmol}) \mathrm{Cul}$ in $50 \mathrm{~mL}$ THF (abs.) $7.55 \mathrm{~mL}(54.2 \mathrm{mmol})$ triethylamine (TEA), $3.0 \mathrm{~mL}(21.66 \mathrm{mmol})$ trimethylsilylacetylene 22 , and $7.2 \mathrm{~mL}(65.0 \mathrm{mmol})$ ethyl chloroglyoxalate 23 were added subsequently under inert gas conditions (dinitrogen). Gas formation occurred upon addition. The reaction mixture was stirred overnight at room temperature. $30 \mathrm{~mL}$ EtOAc were added to the orange suspension. The suspension was washed with $30 \mathrm{~mL}$ sat. aq. $\mathrm{NH}_{4} \mathrm{Cl}$ solution in a separating funnel. The organic phase was separated and washed with $100 \mathrm{~mL}$ water, sat. aq. $\mathrm{NaHCO}_{3}$ solution, and sat. aq. $\mathrm{NaCl}$ solution. The separated aqueous phases were extracted three times, with $30 \mathrm{~mL}$ EtOAc, respectively. The combined organic phases were dried over $\mathrm{Na}_{2} \mathrm{SO}_{4}$ and the solvent was removed under reduced pressure. The crude product was purified by column chromatography with hexane/EtOAc ( $\left.5: 1 ; R_{\mathrm{f}}=0.5\right) .2 .948 \mathrm{~g}(69 \%) 23$ were collected as a pale-yellow liquid.

${ }^{1} \mathrm{H} \mathrm{NMR}\left(400 \mathrm{MHz}, \mathrm{CDCl}_{3}\right): \delta=4.36\left(\mathrm{q},{ }^{3} \mathrm{~J}=7.2 \mathrm{~Hz}, 2 \mathrm{H}\right), 1.39\left(\mathrm{t},{ }^{3} \mathrm{~J}=7.2 \mathrm{~Hz}, 3 \mathrm{H}\right), 0.29(\mathrm{~s}, 9 \mathrm{H}) \mathrm{ppm}$.

${ }^{13} \mathrm{C}\left\{{ }^{1} \mathrm{H}\right\} \mathrm{NMR}\left(101 \mathrm{MHz}, \mathrm{CDCl}_{3}\right): \delta=169.5,159.1,107.0,100.3,63.4,14.1,-0.9 \mathrm{ppm}$.

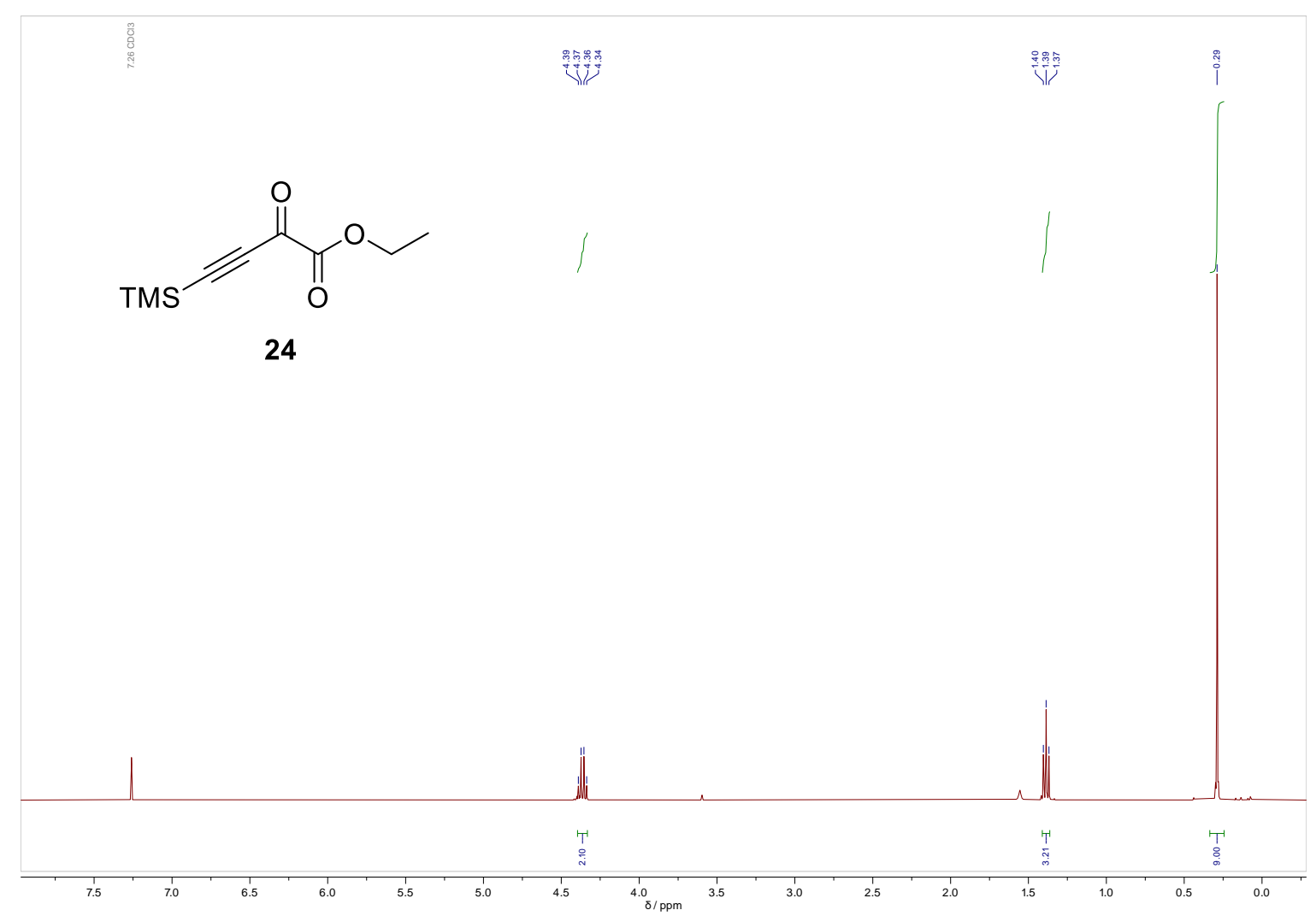

Figure S3: ${ }^{1} \mathrm{H}$ NMR spectrum of trimethylsilylethynylglyoxylic acid ethyl ester (24). 

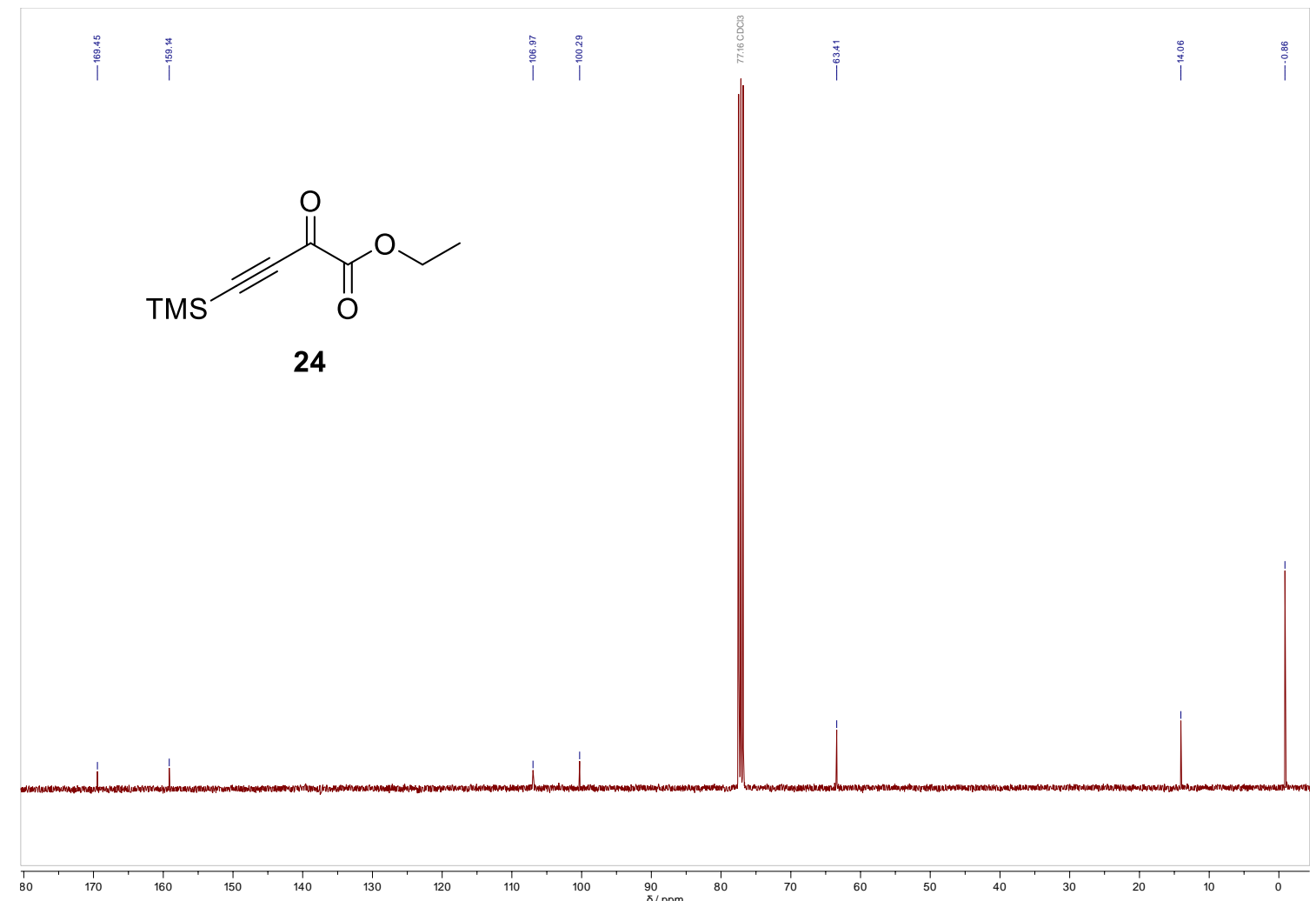

Figure S4: ${ }^{13} \mathrm{C}\left\{{ }^{1} \mathrm{H}\right\}$ NMR spectrum of trimethylsilylethynylglyoxylic acid ethyl ester (24). 


\section{Matrix Isolation}

Equipment used:

- Sumitomo cryostat system consisting of an RDK 408D2 closed-cycle refrigerator cold head and an $\mathrm{F}-70$ compressor unit.

- Voltcraft K204 Digitales/Voltcraft LAP-1363 (25.6 V /1.10 A)

- $\mathrm{Ni} / \mathrm{CrNi}$ thermocouple

- Bruker Vertex 70 FTIR spectrometer

- Jasco V-760 spectrophotometer

- high-pressure mercury lamp (SP200)

A polished CsI window was mounted in the cold head's sample holder. The sample holder, connected with silicon diodes for temperature measurements, was covered by the vacuum shroud, which was equipped with $\mathrm{KBr}$ windows to allow for IR measurements. In some experiments $\mathrm{BaF}_{2}$ windows were used due to their higher transparency when measuring UV/Vis spectra. The sample and the host gas (argon, purity of $99.999 \%$ ) were deposited at $15 \mathrm{~K}$. All spectral data were collected at $3 \mathrm{~K}$, except for one kinetic experiment, which was performed at $20 \mathrm{~K}$. The pyrolysis zone was equipped with a heatable $90 \mathrm{~mm}$ long quartz tube (inner diameter $7 \mathrm{~mm}$ ), controlled by the heating couple (connected to the Voltcraft measuring device). The travel distance of the sample from the pyrolysis zone to the matrix was ca. $45 \mathrm{~mm}$. Argon was stored in a $2 \mathrm{~L}$ gas balloon, which was evacuated and filled three times before every experiment. The sample was stored in a Schlenk tube at $-40{ }^{\circ} \mathrm{C}\left(\mathrm{EtOH} / \mathrm{N}_{2}(\mathrm{l})\right)$ and reduced pressure (ca. $3 \times 10^{-6} \mathrm{mbar}$ ). The deposition was carried out on both sides of the matrix window in the dark (preventing unwanted photochemistry) at a rate of ca. $1 \mathrm{mbar} \mathrm{min}^{-1}$, based on the pressure inside the argon balloon. The deposition was carried out at a pyrolysis temperature of $800{ }^{\circ} \mathrm{C}$ until the strongest band of 1 reached an intensity of ca. 0.4 a.u.. IR spectra were recorded between 7000 and $350 \mathrm{~cm}^{-1}$ with a resolution of $0.7 \mathrm{~cm}^{-1}$. A spectrum of the cold matrix window before deposition was used as background spectrum for the subsequent IR measurements. UV/Vis spectra were recorded between 200 and $900 \mathrm{~nm}$ with a resolution of $1 \mathrm{~nm}$. During the kinetic measurements, the matrix head and the spectrometer were covered by a black blanket to reduce external influences. Note that the internal light source of the spectrometer still had some influence on the measurement as outlined below. The high-pressure-mercury lamp was used for irradiation of the matrix during photochemical experiments.

We performed the irradiation at $436 \mathrm{~nm}$, because absorptions of other hydroxycarbenes lie in this range. For example, for cyanohydroxycarbene (17) a broad experimental absorption was found between 400 and $550 \mathrm{~nm}$ with a maximum at $467 \mathrm{~nm}$, while the computed absorption lies at $531 \mathrm{~nm}{ }^{1}$ For $\mathbf{1 t}$, unfortunately, we were not able to obtain its UV/Vis absorption experimentally. The computed absorption of $\mathbf{1 t}$ is $537 \mathrm{~nm}$ (vide infra) and we assume similar overestimation of this value as for $\mathbf{1 7}$. Herein, we needed rather long irradiation times to induce a (nearly) complete reaction (cf. $\mathbf{1 7}^{1}$ where light from an LED with $\lambda=(520 \pm 50) \mathrm{nm}$ was used). This might be a hint, that $436 \mathrm{~nm}$ is just inside the upper or lower (more likely) margin of the actual absorption. Note that we obtained similar results with optimal irradiation times varying for only a few minutes when irradiating at 254 or $313 \mathrm{~nm}$ instead. Using a green LED (ca. $520 \mathrm{~nm}$ ) resulted in accelerated photoreactivity (10 s to reach the maximum concentration of $\mathbf{1 c}$ and $5 \mathrm{~min}$ for the nearly complete reaction $\mathbf{1} \rightarrow \mathbf{2}$ ). 


\section{Matrix IR Spectra}

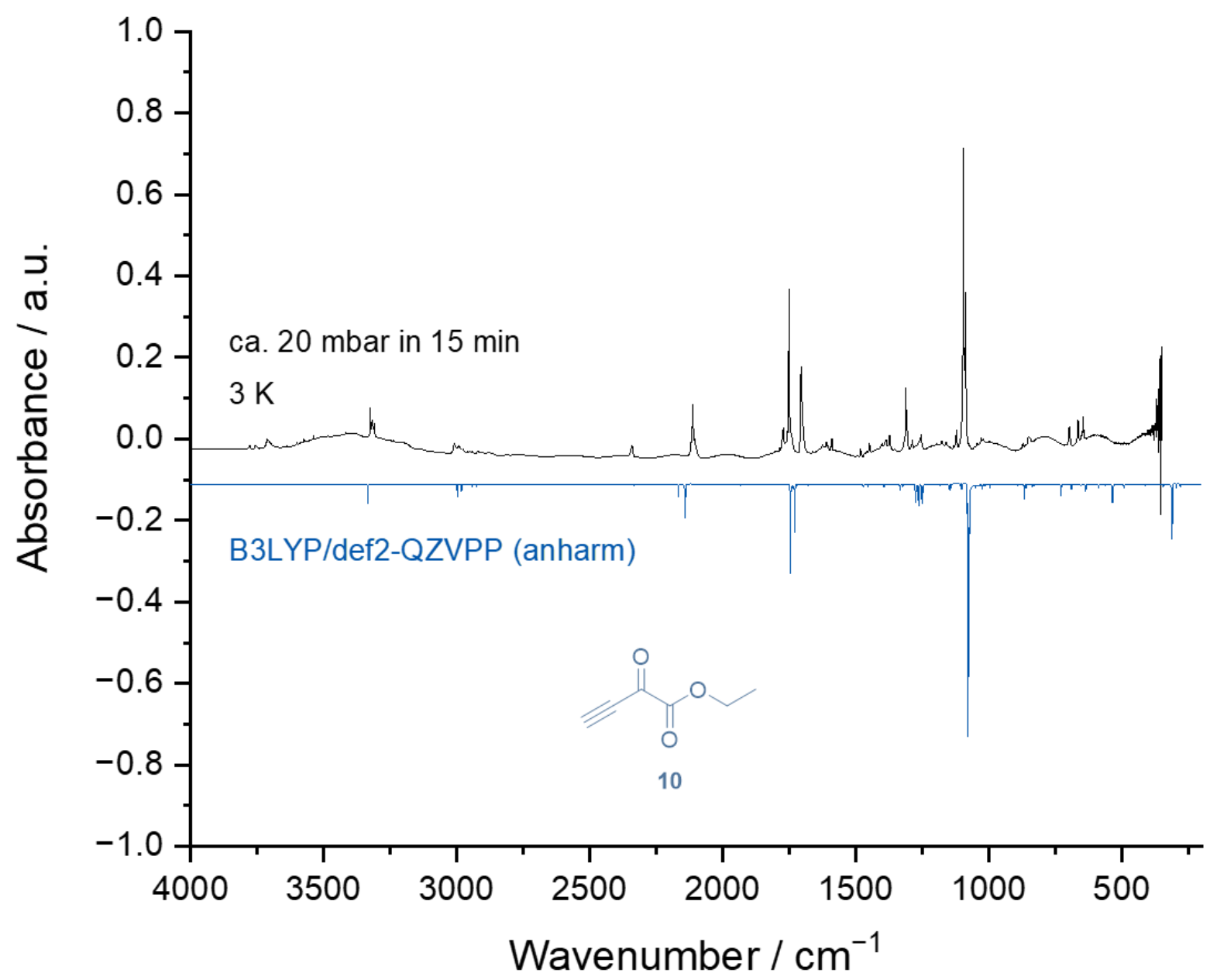

Figure S5: Comparison of the experimental matrix IR spectrum and the computed anharmonic spectrum (B3LYP/def2-QZVPP) of ethynylglyoxylic acid ethyl ester (10). 


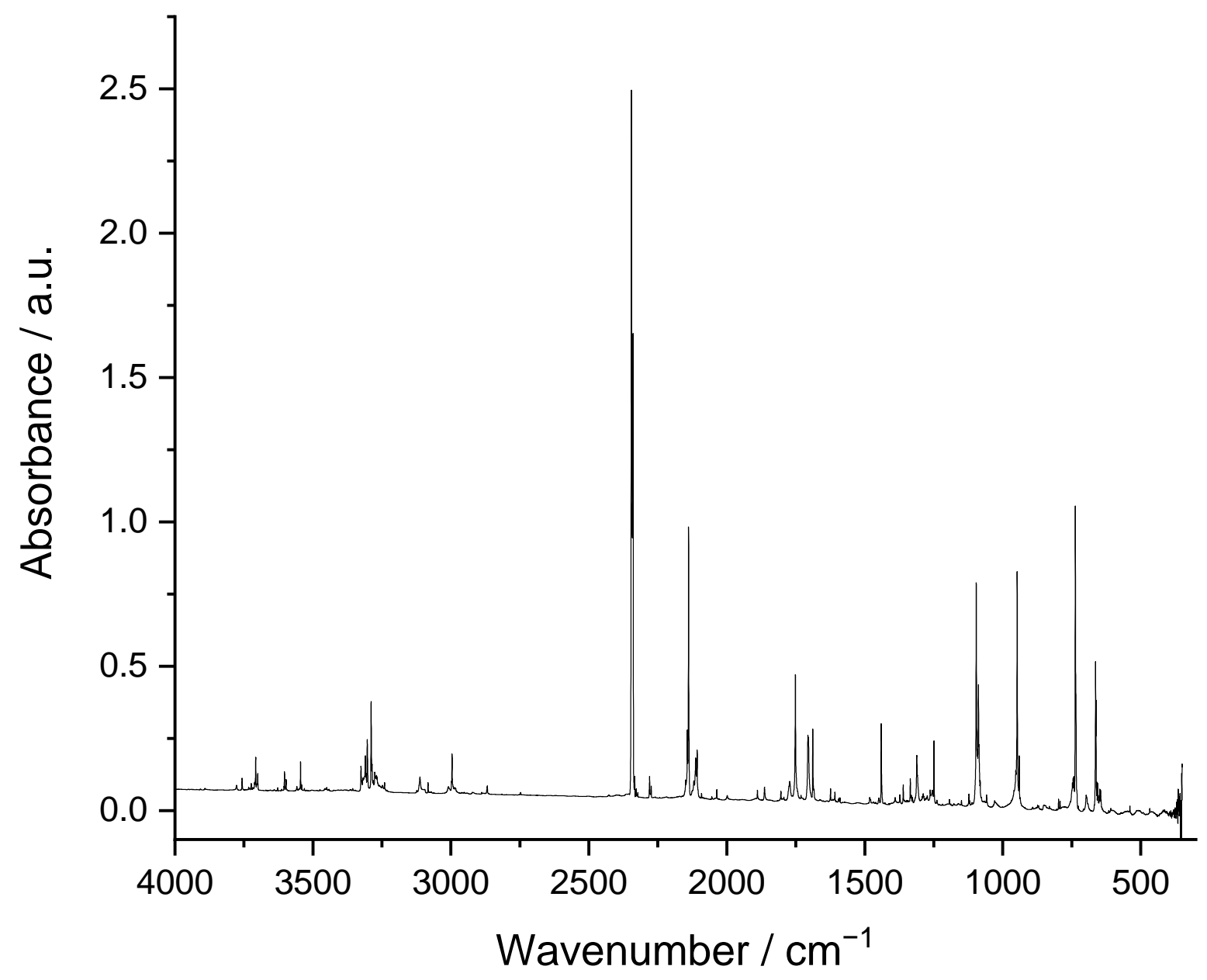

Figure S6: Typical matrix IR spectrum recorded after pyrolysis $\left(900{ }^{\circ} \mathrm{C}\right)$ of ethynylglyoxylic acid ethyl ester (10). Figures S7 to S9 show details of this spectrum with band assignments. 


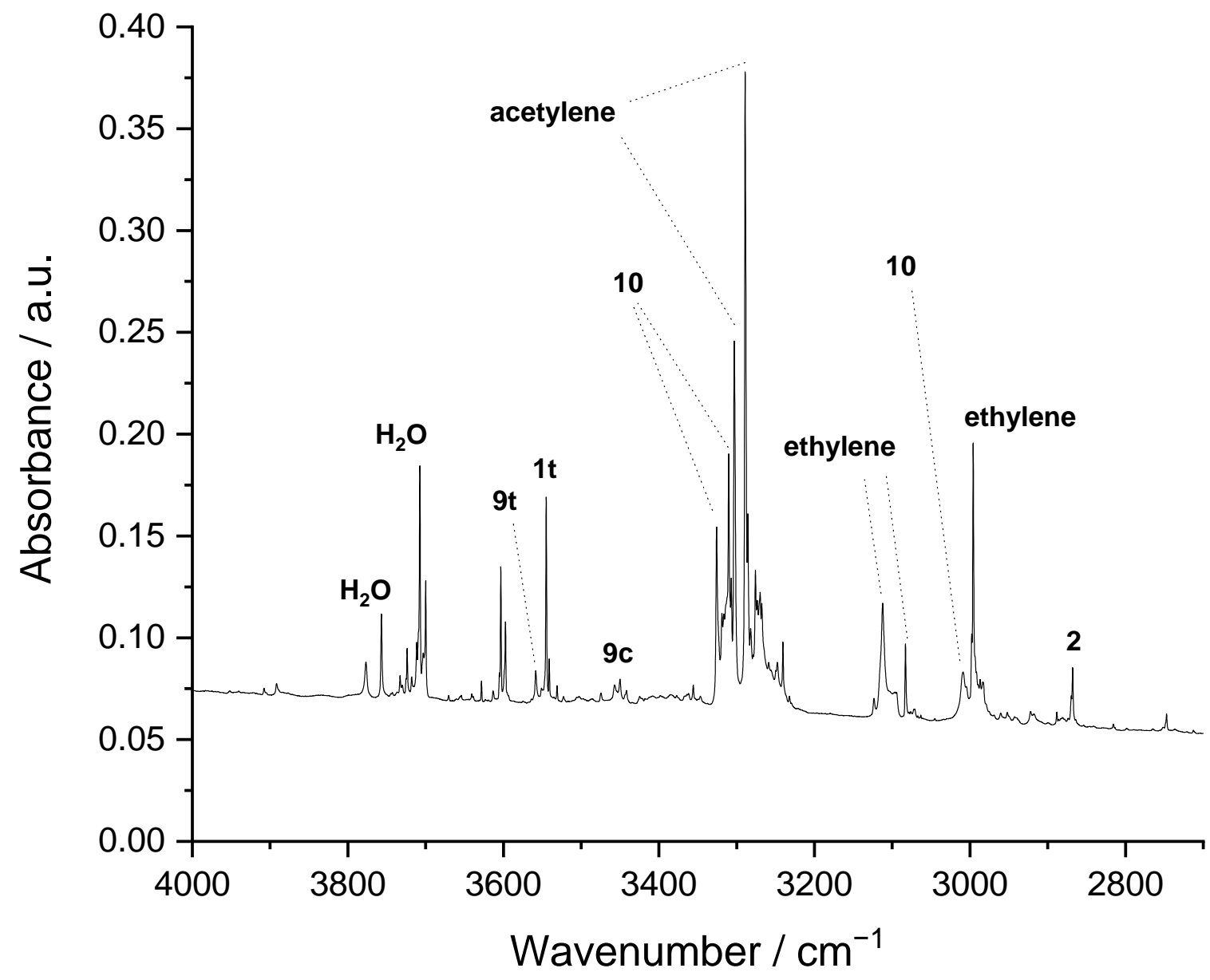

Figure S7: Detailed region (4000 to $2700 \mathrm{~cm}^{-1}$ ) of the spectrum in Figure S6. 


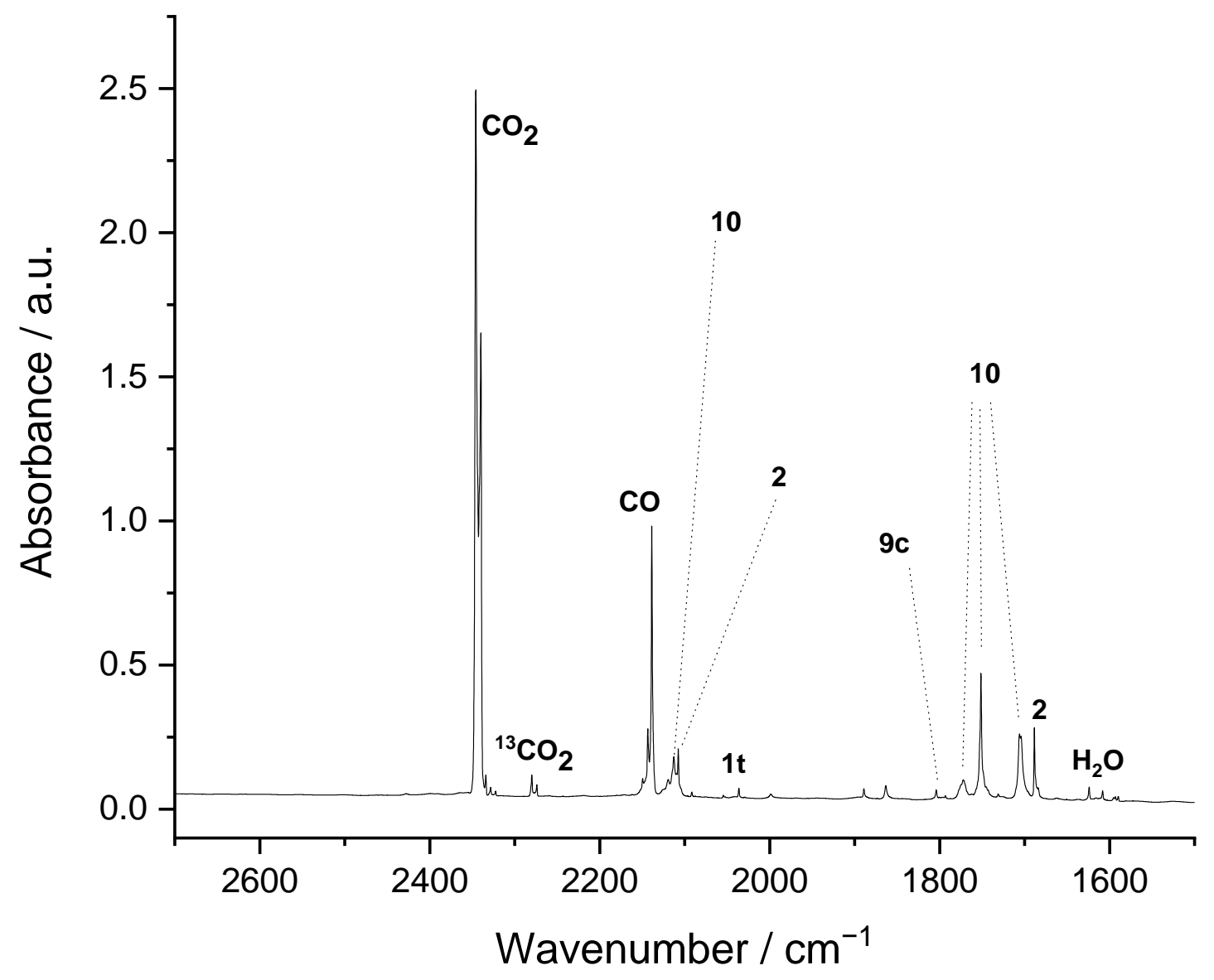

Figure S8: Detailed region (2700 to $1500 \mathrm{~cm}^{-1}$ ) of the spectrum in Figure $S 6$. 


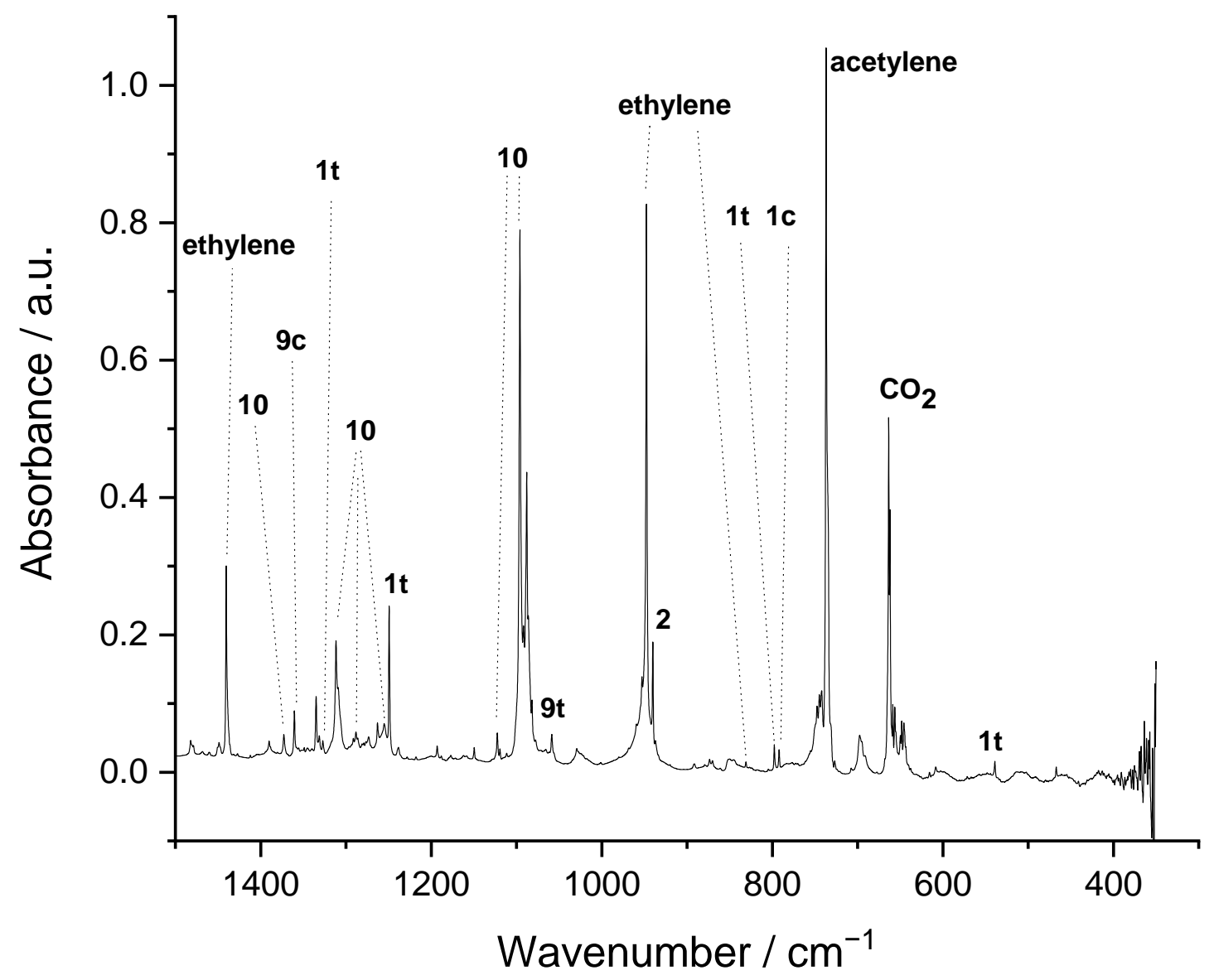

Figure S9: Detailed region $\left(1500\right.$ to $\left.300 \mathrm{~cm}^{-1}\right)$ of the spectrum in Figure $S 6$. 


\section{Matrix IR Assignments}

In Table S1 to S6 we compare observed with computed IR bands. Especially for $\mathbf{1 t}$ and $\mathbf{1 c}$ large anharmonicities for the respective $v(\mathrm{OH})$ vibration are apparent. The anharmonic computed values were used for band assignments. We provide the computed values of the harmonic vibrations as well as these are the basis for ZPVE corrections of electronic energies (vide infra) and comparison purposes. Generally, we find that anharmonic B3LYP IR spectra simulate experimental matrix spectra very reasonably.

Table S1: Comparison of experimental vibrational frequencies of trans-ethynylhydroxycarbene (1t) isolated in an Ar matrix at $3 \mathrm{~K}$ and computed vibrational frequencies at the B3LYP/def2-QZVPP level of theory (unscaled). Only those overtones and combination bands are given, whose computed intensities exceed $10 \mathrm{~km} \mathrm{~mol}^{-1}$.

\begin{tabular}{|c|c|c|c|c|c|c|c|}
\hline Assignment & Sym. & $\tilde{v}_{\text {anharm }} / \mathrm{cm}^{-1}$ & $I_{\mathrm{rel}} / \mathrm{km} \mathrm{mol}^{-1}$ & $\tilde{v}_{\text {harm }} / \mathrm{cm}^{-1}$ & $I_{\mathrm{rel}} / \mathrm{km} \mathrm{mol}^{-1}$ & $v_{\exp .} / \mathrm{cm}^{-1}$ & $I_{\text {rel }}{ }^{[a]}$ \\
\hline$\delta(\mathrm{CCC})$ & $a^{\prime}$ & 239.5 & 12.8 & 241.6 & 12.6 & o.o.r. & o.o.r. \\
\hline$\pi(\mathrm{CCC})$ & $a^{\prime \prime}$ & 267.0 & 10.6 & 267.0 & 10.6 & o.o.r. & o.o.r. \\
\hline$\delta(\mathrm{CH})$ & $a^{\prime}$ & 514.8 & 17.2 & 534.5 & 64.7 & n.o. & n.o. \\
\hline $2 \times \pi(\mathrm{CCC})$ & $a^{\prime}$ & 533.2 & 63.2 & 533.9 & - & 539.0 & $w$ \\
\hline$\delta(\mathrm{CCO})$ & $a^{\prime}$ & 619.5 & 0.8 & 627.0 & 0.8 & n.o. & n.o. \\
\hline$\pi(\mathrm{CH})$ & $a^{\prime \prime}$ & 763.8 & 1.3 & 788.8 & 12.9 & n.o. & n.o. \\
\hline$\pi(\mathrm{CCC})+\delta(\mathrm{CH})$ & $a^{\prime \prime}$ & 791.3 & 24.7 & 801.4 & - & n.o. & n.o. \\
\hline$\pi(\mathrm{OH})$ & $a^{\prime \prime}$ & 806.3 & 62.3 & 818.3 & 73.7 & 797.7 & w \\
\hline$v(\mathrm{C}-\mathrm{C})$ & $a^{\prime}$ & 902.0 & 7.8 & 923.3 & 12.9 & 891.5 & vw \\
\hline $2 \times \delta(\mathrm{CH})$ & $a^{\prime}$ & 1033.5 & 23.2 & 1068.9 & - & n.o. & n.o. \\
\hline $2 \times \delta(\mathrm{CCO})$ & $a^{\prime}$ & 1232.7 & 19.4 & 1254.0 & - & n.o. & n.o. \\
\hline$v(\mathrm{C}-\mathrm{O})$ & $a^{\prime}$ & 1238.4 & 321.2 & 1276.3 & 359.1 & 1249.4 & vs \\
\hline$\delta(\mathrm{COH})$ & $a^{\prime}$ & 1335.2 & 38.8 & 1373.2 & 20.0 & 1327.2 & w \\
\hline$v(\mathrm{C} \equiv \mathrm{C})$ & $a^{\prime}$ & 2069.9 & 19.6 & 2110.5 & 24.8 & 2036.2 & $\mathrm{~m}$ \\
\hline$v(\mathrm{CH})$ & $a^{\prime}$ & 3333.6 & 57.1 & 3458.8 & 65.7 & 3306.9 & $\mathrm{~m}$ \\
\hline$v(\mathrm{OH})$ & $a^{\prime}$ & 3563.1 & 130.3 & 3755.3 & 163.4 & 3545.0 & $\mathrm{~s}$ \\
\hline
\end{tabular}

[a] relative experimental intensities ( $\mathrm{vw}=$ very weak, $\mathrm{w}=$ weak, $\mathrm{m}=$ middle, $\mathrm{s}=\mathrm{strong}, \mathrm{vs}=$ very strong); n.o. = not observed; o.o.r. = out of range. 
Table S2: Comparison of experimental vibrational frequencies of cis-ethynylhydroxycarbene (1c) isolated in an Ar matrix at $3 \mathrm{~K}$ and computed vibrational frequencies at the B3LYP/def2-QZVPP level of theory (unscaled). Only those overtones and combination bands are given, whose computed intensities exceed $10 \mathrm{~km} \mathrm{~mol}^{-1}$.

\begin{tabular}{llllllll}
\hline Assignment & Sym. & $\tilde{v}_{\text {anharm }} / \mathrm{cm}^{-1}$ & $I_{\text {rel }} / \mathrm{km} \mathrm{mol}^{-1}$ & $\tilde{v}_{\text {harm }} / \mathrm{cm}^{-1}$ & $I_{\text {rel }} / \mathrm{km} \mathrm{mol}^{-1}$ & $v_{\text {exp. }} / \mathrm{cm}^{-1}$ & $I_{\text {rel }}^{[\text {[a] }}$ \\
\hline$\delta(\mathrm{CCC})$ & $a^{\prime}$ & 218.8 & 11.9 & 221.4 & 11.6 & o.o.r. & o.o.r. \\
$\pi(\mathrm{CCC})$ & $a^{\prime \prime}$ & 256.5 & 8.1 & 260.9 & 8.7 & o.o.r. & o.o.r. \\
$\delta(\mathrm{CH})$ & $a^{\prime}$ & 518.4 & 66.0 & 546.6 & 61.6 & n.o. & n.o. \\
$\delta(\mathrm{CCO})$ & $a^{\prime}$ & 627.7 & 6.4 & 636.7 & 12.0 & n.o. & n.o. \\
$\pi(\mathrm{CH})$ & $a^{\prime \prime}$ & 759.4 & 20.1. & 783.8 & 26.8 & n.o. & n.o. \\
$\pi(\mathrm{OH})$ & $a^{\prime \prime}$ & 796.8 & 100.9 & 825.9 & 106.5 & 792.1 & w \\
$v(\mathrm{C}-\mathrm{C})$ & $a^{\prime}$ & 877.0 & 2.2 & 898.8 & 2.3 & n.o. & n.o. \\
$2 \times \delta(\mathrm{CH})$ & $a^{\prime}$ & 1037.2 & 11.1 & 1093.2 & - & n.o. & n.o. \\
$v(\mathrm{C}-\mathrm{O})$ & $a^{\prime}$ & 1265.9 & 189.6 & 1309.4 & 251.4 & 1263.0 & $\mathrm{m}$ \\
$\delta(\mathrm{COH})$ & $a^{\prime}$ & 1308.1 & 53.8 & 1345.4 & 2.3 & n.o. & n.o. \\
$v(\mathrm{C} \equiv \mathrm{C})$ & $a^{\prime}$ & 2064.3 & 15.0 & 2105.6 & 18.6 & 2029.1 & vw \\
$v(\mathrm{CH})$ & $a^{\prime}$ & 3324.7 & 35.7 & 3454.6 & 76.3 & n.o. & n.o. \\
$v(\mathrm{OH})$ & $a^{\prime}$ & 3260.6 & 54.3 & 3499.1 & 23.8 & n.o. & n.o. \\
$\delta(\mathrm{COH})+v(\mathrm{C} \equiv \mathrm{C})$ & $a^{\prime}$ & 3361.7 & 28.9 & 3450.9 & - & n.o. & n.o.
\end{tabular}

[a] relative experimental intensities ( $\mathrm{vw}=$ very weak, $\mathrm{w}=$ weak, $\mathrm{m}=$ middle, $\mathrm{s}=\mathrm{strong}, \mathrm{vs}=$ very strong); n.o. = not observed; o.o.r. = out of range. 
Table S3: Comparison of experimental vibrational frequencies of propynal (2) isolated in an Ar matrix at $3 \mathrm{~K}$ and computed vibrational frequencies at the B3LYP/def2-QZVPP level of theory (unscaled). Only those overtones and combination bands are given, whose computed intensities exceed $10 \mathrm{~km} \mathrm{~mol}^{-1}$.

\begin{tabular}{|c|c|c|c|c|c|c|c|}
\hline Assignment & Sym. & $\tilde{v}_{\text {anharm }} / \mathrm{cm}^{-1}$ & $I_{\mathrm{rel}} / \mathrm{km} \mathrm{mol}^{-1}$ & $\tilde{v}_{\text {harm }} / \mathrm{cm}^{-1}$ & $I_{\mathrm{rel}} / \mathrm{km} \mathrm{mol}^{-1}$ & $v_{\text {exp. }} / \mathrm{cm}^{-1}$ & $I_{\text {rel }}{ }^{[\mathrm{a}]}$ \\
\hline$\delta(\mathrm{CCC})$ & $a^{\prime}$ & 218.0 & 5.7 & 214.9 & 6.0 & o.o.r. & o.o.r. \\
\hline$\pi(\mathrm{CCC})$ & $a^{\prime \prime}$ & 273.7 & 9.7 & 273.1 & 9.6 & o.o.r. & o.o.r. \\
\hline$\delta(\mathrm{CCO})$ & $a^{\prime}$ & 628.6 & 12.9 & 634.6 & 8.2 & n.o. & n.o. \\
\hline$\delta(\mathrm{CH})$ & $a^{\prime}$ & 673.9 & 40.6 & 683.7 & 44.4 & 648.2 & w \\
\hline$\pi(\mathrm{CH})$ & $a^{\prime \prime}$ & 735.4 & 33.8 & 744.2 & 32.9 & n.o. & n.o. \\
\hline$v(\mathrm{C}-\mathrm{C})$ & $a^{\prime}$ & 940.9 & 101.3 & 955.9 & 103.4 & 940.1 & $\mathrm{~m}$ \\
\hline$\pi(\mathrm{CHO})$ & $a^{\prime \prime}$ & 996.6 & 0.3 & 1012.0 & 0.2 & n.o. & n.o. \\
\hline$\delta(\mathrm{CHO})$ & $a^{\prime}$ & 1388.4 & 7.6 & 1417.7 & 8.3 & n.o. & n.o. \\
\hline$v(\mathrm{C}=\mathrm{O})$ & $a^{\prime}$ & 1720.0 & 209.9 & 1750.7 & 216.3 & 1688.5 & $\mathrm{~s}$ \\
\hline$v(\mathrm{C} \equiv \mathrm{C})$ & $a^{\prime}$ & 2160.5 & 57.5 & 2195.5 & 62.3 & 2107.5 & $\mathrm{~m}$ \\
\hline$v(\mathrm{CH})$ & $a^{\prime}$ & 2705.7 & 32.0 & 2937.1 & 64.3 & n.o. & n.o. \\
\hline $2 \times \delta(\mathrm{CHO})$ & $a^{\prime}$ & 2833.4 & 39.0 & 2835.3 & - & 2868.0 & w \\
\hline$v(\mathrm{CH})$ & $a^{\prime}$ & 3334.8 & 44.6 & 3457.6 & 49.8 & n.o. & n.o. \\
\hline
\end{tabular}

[a] relative experimental intensities ( $\mathrm{vw}=$ very weak, $\mathrm{w}=$ weak, $\mathrm{m}=$ middle, $\mathrm{s}=\mathrm{strong}, \mathrm{vs}=$ very strong); n.o. = not observed; o.o.r. = out of range. 
Table S4: Comparison of experimental vibrational frequencies of cis-ethynylglyoxylic acid (9c) isolated in an Ar matrix at $3 \mathrm{~K}$ and computed vibrational frequencies at the B3LYP/def2-QZVPP level of theory (unscaled). Only those overtones and combination bands are given, whose computed intensities exceed $10 \mathrm{~km} \mathrm{~mol}^{-1}$.

\begin{tabular}{|c|c|c|c|c|c|c|c|}
\hline Assignment & Sym. & $\tilde{v}_{\text {anharm }} / \mathrm{cm}^{-1}$ & $I_{\text {rel }} / \mathrm{km} \mathrm{mol}^{-1}$ & $\tilde{v}_{\text {harm }} / \mathrm{cm}^{-1}$ & $I_{\text {rel }} / \mathrm{km} \mathrm{mol}^{-1}$ & $v_{\text {exp. }} / \mathrm{cm}^{-1}$ & $I_{\text {rel }}{ }^{[a]}$ \\
\hline$\tau(\mathrm{COOH})$ & $a^{\prime \prime}$ & 70.7 & 6.2 & 78.8 & 6.5 & o.o.r. & o.o.r. \\
\hline$\delta(\mathrm{CCC})$ & $a^{\prime}$ & 136.6 & 5.6 & 138.4 & 6.1 & o.o.r. & o.o.r. \\
\hline$\pi(\mathrm{CCC})$ & $a^{\prime \prime}$ & 228.6 & 0.2 & 232.5 & 0.1 & o.o.r. & o.o.r. \\
\hline$\delta(\mathrm{CCO})$ & $a^{\prime}$ & 288.0 & 33.2 & 295.1 & 34.4 & o.o.r. & o.o.r. \\
\hline$\delta(\mathrm{CCO})$ & $a^{\prime}$ & 417.0 & 1.2 & 428.8 & 2.3 & n.o. & n.o. \\
\hline$\pi(\mathrm{CCO})$ & $a^{\prime \prime}$ & 472.1 & 41.4 & 480.7 & 41.8 & n.o. & n.o. \\
\hline$\delta(\mathrm{CCO})$ & $a^{\prime}$ & 509.7 & 0.2 & 524.5 & 0.3 & n.o. & n.o. \\
\hline$\delta(\mathrm{CCC})+\delta(\mathrm{CCO})$ & $a^{\prime}$ & 650.7 & 19.7 & 662.9 & - & n.o. & n.o. \\
\hline$\pi(\mathrm{OH})$ & $a^{\prime \prime}$ & 650.8 & 89.2 & 702.1 & 83.9 & n.o. & n.o. \\
\hline$\delta(\mathrm{CCO})$ & $a^{\prime}$ & 654.7 & 27.5 & 662.4 & 28.9 & n.o. & n.o. \\
\hline$\delta(\mathrm{CH})$ & $a^{\prime}$ & 675.1 & 15.0 & 687.7 & 34.8 & n.o. & n.o. \\
\hline$v(\mathrm{C}-\mathrm{C})$ & $a^{\prime}$ & 747.5 & 3.6 & 760.4 & 3.8 & n.o. & n.o. \\
\hline$\pi(\mathrm{CH})$ & $a^{\prime \prime}$ & 749.1 & 24.5 & 762.1 & 28.2 & n.o. & n.o. \\
\hline$\pi(\mathrm{CCO})$ & $a^{\prime \prime}$ & 819.5 & 1.0 & 830.9 & 1.8 & n.o. & n.o. \\
\hline$\delta(\mathrm{CCO})+\delta(\mathrm{CCO})$ & $a^{\prime}$ & 1075.0 & 24.4 & 1091.2 & - & n.o. & n.o. \\
\hline$v(\mathrm{C}-\mathrm{C})$ & $a^{\prime}$ & 1077.4 & 175.8 & 1102.4 & 217.1 & 1081.9 & $\mathrm{~m}$ \\
\hline$\pi(\mathrm{CCO})+\pi(\mathrm{OH})$ & $a^{\prime}$ & 1113.1 & 28.7 & 1182.7 & - & n.o. & n.o. \\
\hline$\delta(\mathrm{COH})$ & $a^{\prime}$ & 1182.6 & 76.6 & 1216.5 & 65.3 & 1193.0 & $w$ \\
\hline $2 \times \pi(\mathrm{OH})$ & $a^{\prime}$ & 1262.1 & 37.0 & 1404.2 & - & n.o. & n.o. \\
\hline$\delta(\mathrm{CCO})+v(\mathrm{C}-\mathrm{C})$ & $a^{\prime}$ & 1262.3 & 12.8 & 1285.0 & - & n.o. & n.o. \\
\hline $2 \times \delta(\mathrm{CCO})$ & $a^{\prime}$ & 1339.1 & 152.3 & 1375.5 & - & n.o. & n.o. \\
\hline$\delta(\mathrm{COH})$ & $a^{\prime}$ & 1342.8 & 142.8 & 1374.8 & 360.3 & 1360.5 & $\mathrm{~m}$ \\
\hline$v(\mathrm{C}=\mathrm{O})$ & $a^{\prime}$ & 1702.0 & 154.5 & 1727.8 & 174.1 & n.o. & n.o. \\
\hline$v(\mathrm{C}=\mathrm{O})$ & $a^{\prime}$ & 1812.9 & 204.0 & 1846.6 & 217.3 & 1804.0 & w \\
\hline $2 \times v(C-C)$ & $a^{\prime}$ & 2145.8 & 14.2 & 2204.8 & - & n.o. & n.o. \\
\hline$v(\mathrm{C} \equiv \mathrm{C})$ & $a^{\prime}$ & 2173.1 & 81.7 & 2205.5 & 111.3 & n.o. & n.o. \\
\hline$v(\mathrm{CH})$ & $a^{\prime}$ & 3332.5 & 43.7 & 3456.2 & 53.2 & n.o. & n.o. \\
\hline$v(\mathrm{OH})$ & $a^{\prime}$ & 3442.2 & 99.9 & 3638.8 & 111.5 & 3450.0 & $\mathrm{vw}$ \\
\hline
\end{tabular}

[a] relative experimental intensities ( $\mathrm{vw}=$ very weak, $\mathrm{w}=$ weak, $\mathrm{m}=$ middle, $\mathrm{s}=\mathrm{strong}$, $\mathrm{vs}=$ very strong); n.o. = not observed; o.o.r. = out of range. 
Table S5: Comparison of experimental vibrational frequencies of trans-ethynylglyoxylic acid (9t) isolated in an Ar matrix at $3 \mathrm{~K}$ and computed vibrational frequencies at the B3LYP/def2-QZVPP level of theory (unscaled). Only those overtones and combination bands are given, whose computed intensities exceed $10 \mathrm{~km} \mathrm{~mol}^{-1}$.

\begin{tabular}{|c|c|c|c|c|c|c|c|}
\hline Assignment & Sym. & $\tilde{v}_{\text {anharm }} / \mathrm{cm}^{-1}$ & $I_{\text {rel }} / \mathrm{km} \mathrm{mol}^{-1}$ & $\tilde{v}_{\text {harm }} / \mathrm{cm}^{-1}$ & $I_{\mathrm{rel}} / \mathrm{km} \mathrm{mol}^{-1}$ & $v_{\text {exp. }} / \mathrm{cm}^{-1}$ & $I_{\text {rel }^{[a]}}$ \\
\hline$\tau(\mathrm{COOH})$ & $a^{\prime \prime}$ & 26.3 & 5.8 & 29.4 & 6.2 & o.o.r. & o.o.r. \\
\hline$\delta(\mathrm{CCC})$ & $a^{\prime}$ & 148.3 & 4.6 & 141.5 & 5.7 & o.o.r. & o.o.r. \\
\hline$\pi(\mathrm{CCC})$ & $a^{\prime \prime}$ & 228.8 & 1.1 & 222.8 & 0.8 & o.o.r & o.o.r. \\
\hline$\delta(\mathrm{CCO})$ & $a^{\prime}$ & 282.3 & 5.7 & 282.3 & 6.6 & o.o.r. & o.o.r. \\
\hline$\delta(\mathrm{CCO})$ & $a^{\prime}$ & 428.7 & 5.8 & 432.6 & 8.0 & n.o. & n.o. \\
\hline$\pi(\mathrm{CCO})$ & $a^{\prime \prime}$ & 438.4 & 0.8 & 452.9 & $0.0(3)$ & n.o. & n.o. \\
\hline$\delta(\mathrm{CCO})$ & $a^{\prime}$ & 510.9 & 5.8 & 506.8 & 6.0 & n.o. & n.o. \\
\hline$\pi(\mathrm{OH})$ & $a^{\prime \prime}$ & 603.0 & 142.4 & 642.8 & 140.5 & n.o. & n.o. \\
\hline$\delta(\mathrm{CCO})$ & $a^{\prime}$ & 647.2 & 68.6 & 655.4 & 65.7 & n.o. & n.o. \\
\hline$\delta(\mathrm{CH})$ & $a^{\prime}$ & 679.9 & 21.3 & 685.3 & 34.1 & n.o. & n.o. \\
\hline$v(\mathrm{C}-\mathrm{C})$ & $a^{\prime}$ & 722.6 & 32.0 & 733.7 & 35.4 & n.o. & n.o. \\
\hline$\pi(\mathrm{CH})$ & $a^{\prime \prime}$ & 743.6 & 28.1 & 752.5 & 31.5 & n.o. & n.o. \\
\hline$\pi(\mathrm{CCO})$ & $a^{\prime \prime}$ & 817.8 & $0.0(05)$ & 830.4 & 0.6 & n.o. & n.o. \\
\hline$\delta(\mathrm{CCO})$ & $a^{\prime}$ & 1049.5 & 327.6 & 1074.8 & 373.9 & 1058.6 & $\mathrm{~m}$ \\
\hline$\delta(\mathrm{CCO})+\delta(\mathrm{CCO})$ & $a^{\prime}$ & 1079.5 & 33.4 & 1088.0 & - & n.o. & n.o. \\
\hline$\delta(\mathrm{COH})$ & $a^{\prime}$ & 1149.3 & 32.0 & 1200.2 & 51.5 & n.o. & n.o. \\
\hline $2 \times \pi(\mathrm{OH})$ & $a^{\prime}$ & 1189.9 & 15.2 & 1285.6 & - & n.o. & n.o. \\
\hline$\delta(\mathrm{COH})$ & $a^{\prime}$ & 1339.3 & 12.2 & 1377.6 & 18.0 & n.o. & n.o. \\
\hline $2 \times \delta(\mathrm{CH})$ & $a^{\prime}$ & 1351.2 & 11.6 & 1370.6 & - & n.o. & n.o. \\
\hline$\delta(\mathrm{CH})+\delta(\mathrm{CCO})$ & $a^{\prime}$ & 1726.7 & 11.9 & 1760.1 & - & n.o. & n.o. \\
\hline$v(\mathrm{C}=\mathrm{O})$ & $a^{\prime}$ & 1731.2 & 144.4 & 1757.9 & 176.1 & n.o. & n.o. \\
\hline$\delta(\mathrm{CCO})+\delta(\mathrm{COH})$ & $a^{\prime}$ & 1760.5 & 52.8 & 1810.2 & - & n.o. & n.o. \\
\hline$v(\mathrm{C}-\mathrm{C})+\delta(\mathrm{CCO})$ & $a^{\prime}$ & 1769.7 & 156.6 & 1808.5 & - & 1770.0 & w \\
\hline$v(\mathrm{C}=0)$ & $a^{\prime}$ & 1773.5 & 80.5 & 1806.6 & 251.3 & n.o. & n.o. \\
\hline$v(\mathrm{C} \equiv \mathrm{C})$ & $a^{\prime}$ & 2169.4 & 77.9 & 2202.5 & 94.5 & n.o. & n.o. \\
\hline$v(\mathrm{CH})$ & $a^{\prime}$ & 3335.1 & 45.3 & 3458.4 & 50.9 & n.o. & n.o. \\
\hline$v(\mathrm{OH})$ & $a^{\prime}$ & 3557.0 & 77.4 & 3746.1 & 93.5 & 3558.4 & $w$ \\
\hline
\end{tabular}


Table S6: Comparison of experimental vibrational frequencies of ethynylglyoxylic acid ethyl ester (10) isolated in an Ar matrix at $3 \mathrm{~K}$ and computed vibrational frequencies at the B3LYP/def2-QZVPP level of theory (unscaled). Only those overtones and combination bands are given, whose computed intensities exceed $10 \mathrm{~km} \mathrm{~mol}^{-1}$.

\begin{tabular}{|c|c|c|c|c|c|c|c|}
\hline Assignment & Sym. & $\tilde{v}_{\text {anharm }} / \mathrm{cm}^{-1}$ & $I_{\text {rel }} / \mathrm{km} \mathrm{mol}^{-1}$ & $\tilde{v}_{\text {harm }} / \mathrm{cm}^{-1}$ & $I_{\text {rel }} / \mathrm{km} \mathrm{mol}^{-1}$ & $v_{\text {exp. }} / \mathrm{cm}^{-1}$ & $I_{\mathrm{rel}}{ }^{[\mathrm{a}]}$ \\
\hline$\tau($ COOEt $)$ & $a^{\prime \prime}$ & 46.9 & 1.3 & 17.2 & 4.2 & o.o.r. & o.o.r. \\
\hline$\tau(\mathrm{OEt})$ & $a^{\prime \prime}$ & 83.5 & 7.6 & 65.2 & 1.5 & o.o.r. & o.o.r. \\
\hline$\delta(\mathrm{CCO})$ & $a^{\prime}$ & 109.9 & 5.3 & 106.8 & 4.8 & o.o.r. & o.o.r. \\
\hline$\pi(\mathrm{OEt})$ & $a^{\prime \prime}$ & 138.6 & 0.6 & 122.0 & 0.6 & o.o.r. & o.o.r. \\
\hline$\delta(\mathrm{CCC})$ & $a^{\prime}$ & 172.2 & 0.4 & 174.0 & 0.7 & o.o.r. & o.o.r. \\
\hline$\pi(\mathrm{CCC})$ & $a^{\prime \prime}$ & 227.8 & 0.2 & 229.5 & $0.0(2)$ & o.o.r. & o.o.r. \\
\hline$\tau(\mathrm{Et})$ & $a^{\prime \prime}$ & 265.2 & 0.2 & 259.3 & $0.0(05)$ & o.o.r. & o.o.r. \\
\hline$\delta(\mathrm{CCO})$ & $a^{\prime}$ & 296.1 & 12.3 & 297.5 & 24.2 & o.o.r. & o.o.r. \\
\hline$\tau(\mathrm{OEt})+\pi(\mathrm{CCC})$ & $a^{\prime}$ & 311.9 & 14.1 & 294.7 & - & o.o.r. & o.o.r. \\
\hline$\delta(\mathrm{CCO})$ & $a^{\prime}$ & 359.4 & 1.0 & 364.2 & 1.8 & n.o. & n.o. \\
\hline$\delta(\mathrm{CCO})$ & $a^{\prime}$ & 412.6 & 3.2 & 420.3 & 3.4 & n.o. & n.o. \\
\hline$\delta(\mathrm{CCO})$ & $a^{\prime}$ & 483.7 & 0.2 & 508.8 & 2.3 & n.o. & n.o. \\
\hline$\pi(\mathrm{CCO})$ & $a^{\prime \prime}$ & 494.0 & 17.0 & 517.7 & 30.8 & n.o. & n.o. \\
\hline$\tau(\mathrm{COOEt})+\delta(\mathrm{CCO})$ & $a^{\prime \prime}$ & 536.2 & 15.4 & 526.0 & - & n.o. & n.o. \\
\hline$\delta(\mathrm{C} \equiv \mathrm{CH})$ & $a^{\prime}$ & 637.9 & 43.9 & 677.1 & 53.2 & 645.8 & w \\
\hline$\delta(\mathrm{C} \equiv \mathrm{CH})$ & $a^{\prime}$ & 691.6 & 13.7 & 683.1 & 6.5 & 664.9 & $\mathrm{~m}$ \\
\hline$\pi(\mathrm{C} \equiv \mathrm{CH})$ & $a^{\prime \prime}$ & 730.4 & 33.4 & 749.6 & 30.1 & 697.4 & $\mathrm{~m}$ \\
\hline$\pi(\mathrm{Et})$ & $a^{\prime \prime}$ & 805.6 & 0.4 & 812.1 & 0.6 & n.o. & n.o. \\
\hline$\pi(\mathrm{CCO})$ & $a^{\prime \prime}$ & 824.1 & 1.0 & 833.9 & 0.1 & n.o. & n.o. \\
\hline$\delta(\mathrm{CCO})$ & $a^{\prime}$ & 843.5 & 2.9 & 856.0 & 18.0 & 850.5 & w \\
\hline$\delta(\mathrm{CCO})$ & $a^{\prime}$ & 861.2 & 13.8 & 876.5 & 9.0 & 873.6 & w \\
\hline$v(\mathrm{C}-\mathrm{C})$ & $a^{\prime}$ & 996.6 & 9.0 & 1026.1 & 5.1 & 1001.1 & vw \\
\hline$v(\mathrm{C}-\mathrm{C})$ & $a^{\prime}$ & 1076.3 & 349.5 & 1105.0 & 530.8 & 1088.0 & vs \\
\hline$\delta(\mathrm{CCO})+\delta(\mathrm{C} \equiv \mathrm{CH})$ & $a^{\prime}$ & 1079.3 & 158.6 & 1103.4 & - & 1096.9 & vs \\
\hline$\tau(\mathrm{Et})+\pi(\mathrm{CCO})$ & $a^{\prime}$ & 1082.7 & 20.3 & 1093.2 & - & n.o. & n.o. \\
\hline$v(\mathrm{C}-\mathrm{C})$ & $a^{\prime}$ & 1110.3 & 5.8 & 1136.7 & 12.1 & 1119.4 & vw \\
\hline$\pi(\mathrm{Et})$ & $a^{\prime \prime}$ & 1152.2 & 3.0 & 1178.9 & 3.9 & 1122.5 & w \\
\hline$\delta(\mathrm{CCO})+\delta(\mathrm{CCO})$ & $a^{\prime}$ & 1253.6 & 26.2 & 1276.3 & - & 1255.0 & w \\
\hline$v(\mathrm{C}-\mathrm{C})$ & $a^{\prime}$ & 1265.9 & 106.4 & 1302.8 & 189.1 & 1288.2 & w \\
\hline$\pi(\mathrm{Et})$ & $a^{\prime \prime}$ & 1270.6 & 0.9 & 1301.1 & 1.3 & n.o. & n.o. \\
\hline$\delta(\mathrm{CCO})+\delta(\mathrm{CCO})$ & $a^{\prime}$ & 1276.5 & 37.1 & 1296.8 & - & 1311.6 & s \\
\hline
\end{tabular}




\begin{tabular}{|c|c|c|c|c|c|c|c|}
\hline$\delta(\mathrm{Et})$ & $a^{\prime}$ & 1377.0 & 0.4 & 1404.4 & 0.4 & 1372.8 & $w$ \\
\hline$\delta(\mathrm{Et})$ & $a^{\prime}$ & 1394.8 & 7.9 & 1429.1 & 10.5 & 1391.1 & vw \\
\hline$\pi(\mathrm{Et})$ & $a^{\prime \prime}$ & 1442.5 & 2.5 & 1487.5 & 6.7 & 1448.7 & w \\
\hline$\delta(\mathrm{Et})$ & $a^{\prime}$ & 1450.4 & 2.4 & 1499.3 & 2.1 & 1468.4 & vw \\
\hline$\delta(\mathrm{Et})$ & $a^{\prime}$ & 1474.3 & 5.0 & 1517.8 & 7.9 & 1482.1 & $w$ \\
\hline$v(\mathrm{C}=\mathrm{O})$ & $a^{\prime}$ & 1729.7 & 132.5 & 1755.9 & 159.8 & 1704.0 & s \\
\hline$v(\mathrm{C}=\mathrm{O})$ & $a^{\prime}$ & 1747.9 & 216.8 & 1781.0 & 241.5 & 1751.5 & vs \\
\hline$\delta(\mathrm{C} \equiv \mathrm{CH})+\delta(\mathrm{Et})$ & $a^{\prime}$ & 2141.8 & 70.9 & 2200.9 & - & 2112.8 & $s$ \\
\hline$v(\mathrm{C} \equiv \mathrm{C})$ & $a^{\prime}$ & 2168.8 & 28.6 & 2200.7 & 92.2 & 2138.8 & $w$ \\
\hline$v_{s}\left(\mathrm{CH}_{2}\right)$ & $a^{\prime}$ & 2887.4 & 4.7 & 3052.5 & 11.2 & 2922.2 & $w$ \\
\hline$v_{\text {as }}(\mathrm{Et})$ & $a^{\prime \prime}$ & 2928.9 & 3.4 & 3088.3 & 2.9 & 2952.0 & vw \\
\hline$v_{s}\left(\mathrm{CH}_{3}\right)$ & $a^{\prime}$ & 2944.8 & 10.1 & 3040.9 & 13.6 & 2960.5 & vw \\
\hline$V_{\text {as }}\left(\mathrm{CH}_{3}\right)$ & $a^{\prime}$ & 2968.3 & 4.0 & 3106.4 & 18.3 & 2979.1 & vw \\
\hline $2 x \delta(E t)$ & $a^{\prime}$ & 2982.5 & 18.9 & 3035.5 & - & 2993.6 & vw \\
\hline$v_{\text {as }}(\mathrm{Et})$ & $a^{\prime \prime}$ & 2998.5 & 29.5 & 3118.1 & 27.9 & 3008.9 & w \\
\hline$v(\mathrm{CH})$ & $a^{\prime}$ & 3335.5 & 44.3 & 3459.5 & 52.1 & 3325.8 & $\mathrm{~m}$ \\
\hline
\end{tabular}




\section{Matrix UV/Vis Spectra}

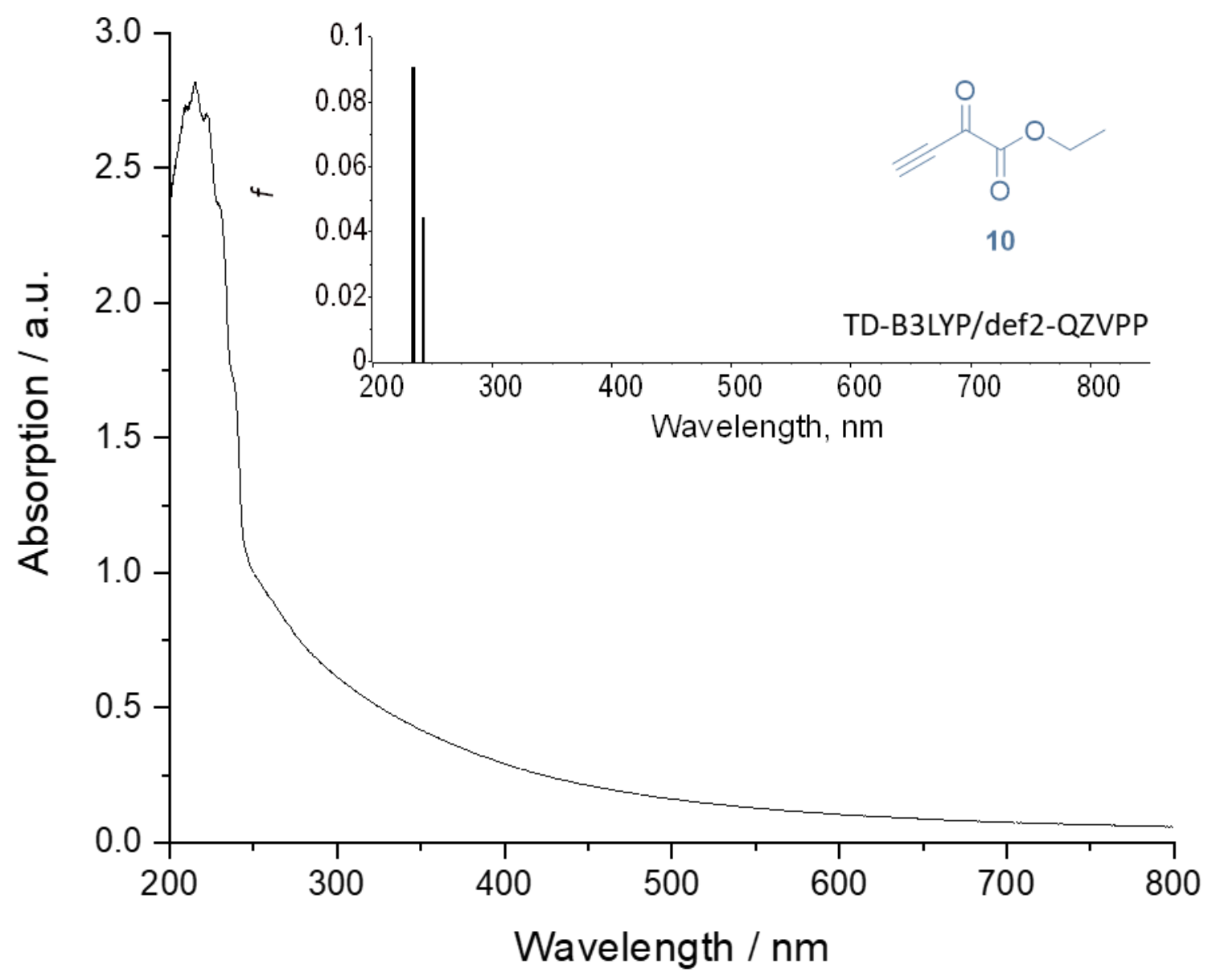

Figure S10: Matrix UV/Vis spectrum measured after deposition of ethynylglyoxylic acid ethyl ester (10) on a cold $\mathrm{BaF}_{2}$ together with an excess of argon. 


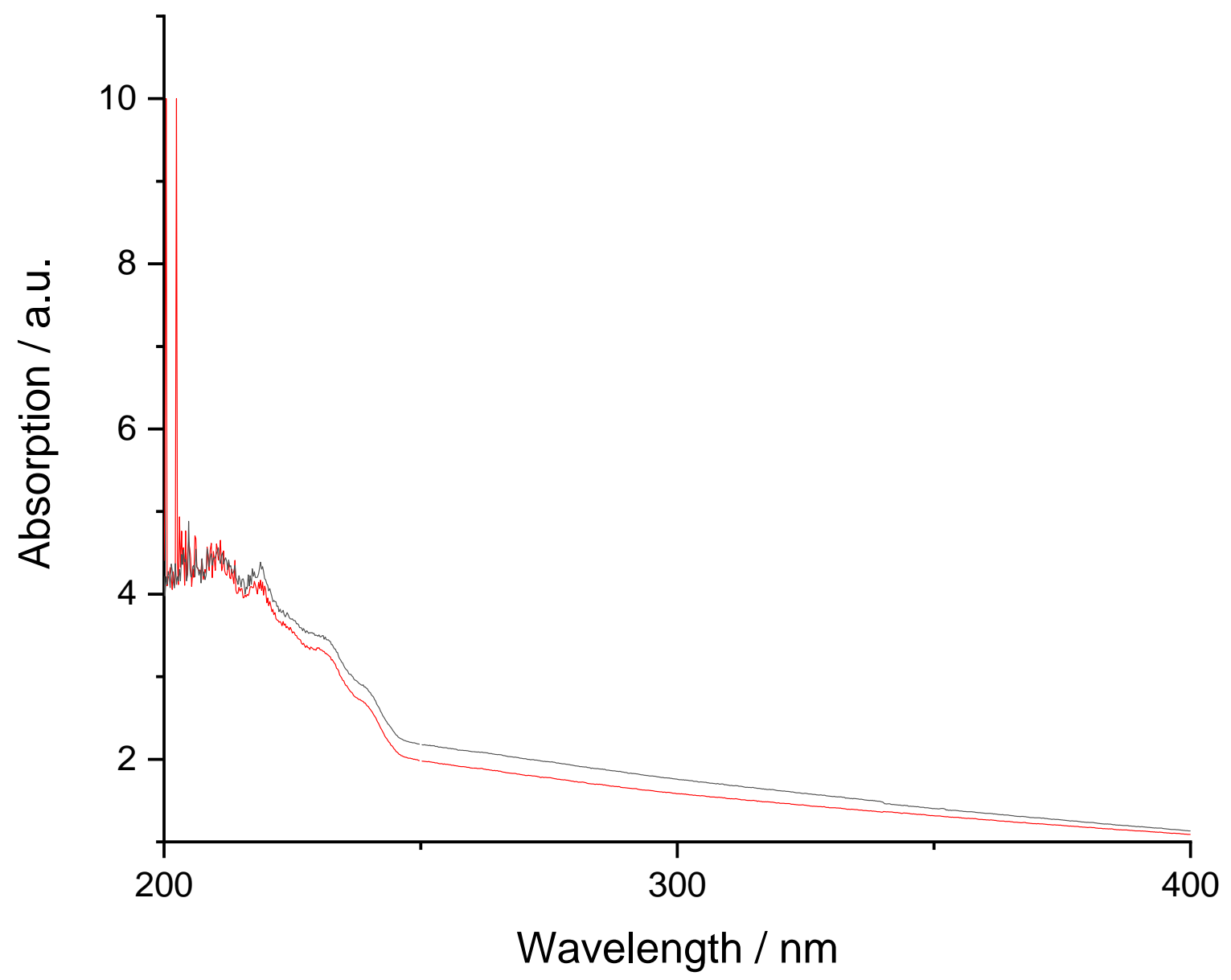

Figure S11: Matrix UV/Vis spectra measured after pyrolysis of 10 at $900{ }^{\circ} \mathrm{C}$ (red) and after 40 min of irradiation at $436 \mathrm{~nm}$ (black) of the obtained matrix. No changes in the spectra can be observed, which is in accord with very minute intensities of the absorptions in TD-B3LYP/def2-QZVPP spectra of $\mathbf{1 t}, \mathbf{1} \mathbf{c}$, and 2. 


\section{Computed UV/Vis Absorptions}

Table S7: TD-B3LYP/def2-QZVPP computed vertical excitation energies of transethynylhydroxycarbene $\mathbf{1 t}$.

\begin{tabular}{|c|c|c|}
\hline Excitation Energy $\lambda / \mathrm{nm}$ & Oscillator Strength $(f)$ & Transition \\
\hline 537.31 & 0.0032 & HOMO $\rightarrow$ LUMO \\
\hline 271.81 & 0.0007 & HOMO-2 $\rightarrow$ LUMO \\
\hline \multirow[t]{2}{*}{260.67} & 0.0090 & HOMO-1 $\rightarrow$ LUMO (23\%) \\
\hline & & HOMO $\rightarrow$ LUMO+1 (77\%) \\
\hline \multirow[t]{5}{*}{223.08} & 0.0968 & HOMO-2 $\rightarrow$ LUMO+1 (5\%) \\
\hline & & HOMO-1 $\rightarrow$ LUMO (49\%) \\
\hline & & HOMO $\rightarrow$ LUMO+1 (17\%) \\
\hline & & HOMO $\rightarrow$ LUMO+2 (24\%) \\
\hline & & HOMO $\rightarrow$ LUMO+3 (5\%) \\
\hline \multirow{4}{*}{190.51} & 0.0691 & HOMO-2 $\rightarrow$ LUMO+1 (3\%) \\
\hline & & HOMO-1 $\rightarrow$ LUMO (6\%) \\
\hline & & HOMO $\rightarrow$ LUMO+2 (55\%) \\
\hline & & HOMO $\rightarrow$ LUMO+3 (36\%) \\
\hline \multirow[t]{5}{*}{183.74} & 0.1874 & HOMO-2 $\rightarrow$ LUMO+1 (9\%) \\
\hline & & HOMO-1 $\rightarrow$ LUMO (11\%) \\
\hline & & HOMO $\rightarrow$ LUMO+2 (17\%) \\
\hline & & HOMO $\rightarrow$ LUMO+3 (59\%) \\
\hline & & HOMO $\rightarrow$ LUMO+5 (4\%) \\
\hline
\end{tabular}

Table S8: TD-B3LYP/def2-QZVPP computed vertical excitation energies of cis-ethynylhydroxycarbene 1c.

\begin{tabular}{|c|c|c|}
\hline Excitation Energy $\lambda / \mathrm{nm}$ & Oscillator Strength $(f)$ & Transition \\
\hline 567.71 & 0.0036 & HOMO $\rightarrow$ LUMO \\
\hline 270.39 & 0.0011 & HOMO-1 $\rightarrow$ LUMO \\
\hline \multirow[t]{2}{*}{266.44} & 0.0272 & HOMO-2 $\rightarrow$ LUMO (17\%) \\
\hline & & HOMO $\rightarrow$ LUMO+1 (83\%) \\
\hline \multirow[t]{5}{*}{218.77} & 0.1386 & HOMO-2 $\rightarrow$ LUMO (57\%) \\
\hline & & HOMO-1 $\rightarrow$ LUMO+1 (12\%) \\
\hline & & HOMO $\rightarrow$ LUMO+1 (14\%) \\
\hline & & HOMO $\rightarrow$ LUMO+2 (13\%) \\
\hline & & HOMO $\rightarrow$ LUMO+3 (4\%) \\
\hline \multirow[t]{4}{*}{191.45} & 0.0778 & HOMO-2 $\rightarrow$ LUMO (4\%) \\
\hline & & HOMO-1 $\rightarrow$ LUMO+1 (4\%) \\
\hline & & HOMO $\rightarrow$ LUMO+2 (73\%) \\
\hline & & HOMO $\rightarrow$ LUMO+3 (19\%) \\
\hline \multirow[t]{4}{*}{184.26} & 0.1691 & HOMO-2 $\rightarrow$ LUMO (4\%) \\
\hline & & HOMO-1 $\rightarrow$ LUMO+1 (10\%) \\
\hline & & HOMO $\rightarrow$ LUMO+2 (11\%) \\
\hline & & HOMO $\rightarrow$ LUMO+3 (75\%) \\
\hline
\end{tabular}


Table S9: TD-B3LYP/def2-QZVPP computed vertical excitation energies of propynal $\mathbf{2}$.

\begin{tabular}{lll}
\hline Excitation Energy $\lambda / \mathrm{nm}$ & Oscillator Strength $(f)$ & Transition \\
\hline 330.97 & 0.0002 & HOMO $\rightarrow$ LUMO \\
259.39 & 0.0000 & HOMO $-1 \rightarrow$ LUMO \\
216.41 & 0.1169 & HOMO $-2 \rightarrow$ LUMO (84\%) \\
& & HOMO-1 $\rightarrow$ LUMO +1 (16\%) \\
182.76 & 0.0039 & HOMO $\rightarrow$ LUMO+1 \\
177.57 & 0.0000 & HOMO $-2 \rightarrow$ LUMO +1 \\
157.44 & 0.0382 & HOMO $\rightarrow$ LUMO+2 (80\%) \\
& & HOMO $\rightarrow$ LUMO+3 (20\%) \\
\hline
\end{tabular}

Table S10: TD-B3LYP/def2-QZVPP computed vertical excitation energies of trans-ethynylglyoxylic acid 9t.

\begin{tabular}{lll}
\hline Excitation Energy $\lambda / \mathrm{nm}$ & Oscillator Strength $(f)$ & Transition \\
\hline 401.32 & 0.0000 & HOMO $\rightarrow$ LUMO \\
291.01 & 0.0000 & HOMO $-1 \rightarrow$ LUMO \\
259.11 & 0.0000 & HOMO $3 \rightarrow$ LUMO (96\%) \\
& & HOMO $\rightarrow$ LUMO+1 (4\%) \\
243.20 & 0.0948 & HOMO $-2 \rightarrow$ LUMO (94\%) \\
& & HOMO $-1 \rightarrow$ LUMO+2 (6\%) \\
210.78 & 0.0397 & HOMO-4 $\rightarrow$ LUMO \\
196.49 & 0.0001 & HOMO-3 $\rightarrow$ LUMO (4\%) \\
& & HOMO $\rightarrow$ LUMO $1(96 \%)$ \\
\hline
\end{tabular}

Table S11: TD-B3LYP/def2-QZVPP computed vertical excitation energies of cis-ethynylglyoxylic acid 9c.

\begin{tabular}{lll}
\hline Excitation Energy $\lambda / \mathrm{nm}$ & Oscillator Strength $(f)$ & Transition \\
\hline 404.78 & 0.0000 & HOMO $\rightarrow$ LUMO \\
300.00 & 0.0000 & HOMO $-1 \rightarrow$ LUMO \\
253.82 & 0.0000 & HOMO-4 $\rightarrow$ LUMO (97\%) \\
& & HOMO $\rightarrow$ LUMO+2 (3\%) \\
242.50 & 0.0848 & HOMO-3 $\rightarrow$ LUMO (5\%) \\
& & HOMO-2 $\rightarrow$ LUMO (90\%) \\
& & HOMO-1 $\rightarrow$ LUMO+1 (5\%) \\
226.08 & 0.0603 & HOMO-3 $\rightarrow$ LUMO (95\%) \\
& & HOMO-2 $\rightarrow$ LUMO (5\%) \\
196.28 & 0.0001 & HOMO-4 $\rightarrow$ LUMO (4\%) \\
& & HOMO $\rightarrow$ LUMO+2 (96\%) \\
\hline
\end{tabular}


Table S12: TD-B3LYP/def2-QZVPP computed vertical excitation energies of ethynylglyoxylic acid ethyl ester 10.

\begin{tabular}{|c|c|c|}
\hline Excitation Energy $\lambda / \mathrm{nm}$ & Oscillator Strength $(f)$ & Transition \\
\hline 401.57 & 0.0000 & HOMO $\rightarrow$ LUMO \\
\hline 287.94 & 0.0000 & HOMO-1 $\rightarrow$ LUMO \\
\hline \multirow[t]{2}{*}{260.27} & 0.0000 & HOMO-4 $\rightarrow$ LUMO (97\%) \\
\hline & & HOMO $\rightarrow$ LUMO+1 (3\%) \\
\hline \multirow[t]{4}{*}{242.13} & 0.0448 & HOMO-3 $\rightarrow$ LUMO (4\%) \\
\hline & & HOMO-2 $\rightarrow$ LUMO (89\%) \\
\hline & & HOMO-1 $\rightarrow$ LUMO+2 (4\%) \\
\hline & & HOMO $\rightarrow$ LUMO+2 (3\%) \\
\hline \multirow[t]{2}{*}{233.16} & 0.0907 & HOMO-3 $\rightarrow$ LUMO (95\%) \\
\hline & & HOMO-2 $\rightarrow$ LUMO (5\%) \\
\hline \multirow[t]{2}{*}{198.39} & 0.0000 & HOMO-9 $\rightarrow$ LUMO (3\%) \\
\hline & & HOMO-5 $\rightarrow$ LUMO (97\%) \\
\hline
\end{tabular}

Table S13: TD-B3LYP/def2-QZVPP computed vertical excitation energies of ethylene.

\begin{tabular}{lll}
\hline Excitation Energy $\lambda / \mathrm{nm}$ & Oscillator Strength $(f)$ & Transition \\
\hline 165.69 & 0.0544 & HOMO $\rightarrow$ LUMO+1 \\
162.32 & 0.3516 & HOMO $\rightarrow$ LUMO \\
154.64 & 0.0000 & HOMO $-1 \rightarrow$ LUMO (89\%) \\
& & HOMO $\rightarrow$ LUMO+3 (11\%) \\
152.61 & 0.0000 & HOMO $\rightarrow$ LUMO+2 \\
150.81 & 0.0000 & HOMO $-1 \rightarrow$ LUMO (10\%) \\
& & HOMO $\rightarrow$ LUMO+3 (90\%) \\
128.65 & 0.0000 & HOMO $1 \rightarrow$ LUMO+1 \\
\hline
\end{tabular}

Table S14: TD-B3LYP/def2-QZVPP computed vertical excitation energies of $\mathrm{CO}_{2}$.

\begin{tabular}{lll}
\hline Excitation Energy $\lambda / \mathrm{nm}$ & Oscillator Strength $(f)$ & Transition \\
\hline 140.41 & 0.0000 & HOMO $-1 \rightarrow$ LUMO+1 (50\%) \\
& & HOMO $\rightarrow$ LUMO+2 (50\%) \\
136.23 & 0.0000 & HOMO $-1 \rightarrow$ LUMO+1 (50\%) \\
& & HOMO $\rightarrow$ LUMO+2 (50\%) \\
136.23 & 0.0000 & HOMO $-1 \rightarrow$ LUMO+2 (50\%) \\
& & HOMO $\rightarrow$ LUMO+1 (50\%) \\
135.42 & 0.0000 & HOMO $\rightarrow$ LUMO \\
135.42 & 0.0000 & HOMO $-1 \rightarrow$ LUMO \\
102.24 & 0.0000 & HOMO $-1 \rightarrow$ LUMO+3 (50\%) \\
& & HOMO $\rightarrow$ LUMO+4 (50\%) \\
\hline
\end{tabular}


Table S15: TD-B3LYP/def2-QZVPP computed vertical excitation energies of acetylene.

\begin{tabular}{lll}
\hline Excitation Energy $\lambda / \mathrm{nm}$ & Oscillator Strength $(f)$ & Transition \\
\hline 183.47 & 0.0000 & HOMO $-1 \rightarrow$ LUMO $(50 \%)$ \\
& & HOMO $\rightarrow$ LUMO+1 $(50 \%)$ \\
178.98 & 0.0000 & HOMO $-1 \rightarrow$ LUMO (50\%) \\
& & HOMO $\rightarrow$ LUMO+1 (50\%) \\
178.98 & 0.0000 & HOMO $-1 \rightarrow$ LUMO+1 (50\%) \\
& & HOMO $\rightarrow$ LUMO (50\%) \\
148.64 & 0.0000 & HOMO $\rightarrow$ LUMO+2 \\
148.64 & 0.0000 & HOMO $-1 \rightarrow$ LUMO +2 \\
146.38 & 0.0709 & HOMO-1 $\rightarrow$ LUMO+3 \\
\hline
\end{tabular}

Table S16: TD-B3LYP/def2-QZVPP computed vertical excitation energies of CO.

\begin{tabular}{lll}
\hline Excitation Energy $\lambda / \mathrm{nm}$ & Oscillator Strength $(f)$ & Transition \\
\hline 146.55 & 0.0844 & HOMO $\rightarrow$ LUMO+1 \\
146.55 & 0.0844 & HOMO $\rightarrow$ LUMO \\
126.34 & 0.0000 & HOMO $-2 \rightarrow$ LUMO+1 $(50 \%)$ \\
& & HOMO $-1 \rightarrow$ LUMO (50\%) \\
122.45 & 0.0000 & HOMO $-2 \rightarrow$ LUMO+1 (50\%) \\
& & HOMO $-1 \rightarrow$ LUMO (50\%) \\
122.45 & 0.0000 & HOMO $-2 \rightarrow$ LUMO (50\%) \\
& & HOMO- $\rightarrow$ LUMO+1 (50\%) \\
104.06 & 0.0723 & HOMO $\rightarrow$ LUMO+2 (96\%) \\
& & HOMO $\rightarrow$ LUMO+3 (4\%) \\
\hline
\end{tabular}

Table S17: TD-B3LYP/def2-QZVPP computed vertical excitation energies of water.

\begin{tabular}{lll}
\hline Excitation Energy $\lambda / \mathrm{nm}$ & Oscillator Strength $(f)$ & Transition \\
\hline 168.07 & 0.0495 & HOMO $\rightarrow$ LUMO \\
137.06 & 0.0000 & HOMO $\rightarrow$ LUMO+1 \\
129.88 & 0.0973 & HOMO $1 \rightarrow$ LUMO \\
110.14 & 0.0348 & HOMO-1 $\rightarrow$ LUMO+1 \\
92.67 & 0.0001 & HOMO $\rightarrow$ LUMO+2 \\
92.50 & 0.1675 & HOMO-2 $\rightarrow$ LUMO \\
\hline
\end{tabular}




\section{Kinetic Analyses}

The kinetics of the reactions $\mathbf{1 t} \rightarrow \mathbf{2}$ and $\mathbf{9 t} \rightarrow \mathbf{9 c}$ were recorded in three individual experiments:

A: Matrix at $3 \mathrm{~K}$, no cut-off filter between spectrometer and matrix window, 294 scans

B: Matrix at $20 \mathrm{~K}$, no cut-off filter between spectrometer and matrix window, 359 scans

C: Matrix at $3 \mathrm{~K}, 4.5 \mu \mathrm{m}$ cut-off filter between spectrometer and matrix window, 410 scans

IR measurements were repeated every hour in each experiment. Note that in the first experiment there is a $19 \mathrm{~h}$ break between the $124^{\text {th }}$ and the $125^{\text {th }}$ scan due to a malfunction of the spectrometer.

Distinguished IR bands were evaluated individually with a self-programmed python script (available at https://github.com/prs-group/Spectra_Analyzer). ${ }^{2-5}$ This script fits the band's profile applying Simpson's rule as implemented in scipy.integrate.simps and integrates between this fit curve and a straight line between two points chosen manually on each side of the respective band. The values of these integrals are plotted against time in Figures S12 to S17. The obtained curves were fitted to monoexponential functions of the form $y=a \cdot \exp (-k \cdot x)+b$ for decaying and $y=a \cdot(1-\exp (-k \cdot x))+b$ for growing bands optimizing the fit parameters $a$ and $b$ in the same python script with the integral values as the ordinate and time as the abscissa. The tunneling half-life $\left(t_{1 / 2}\right)$ was obtained by $t_{1 / 2}=\ln (2) / \mathrm{k}$ from the rate constant $\mathrm{k}$. The errors of the mean value of $t_{1 / 2}$ were estimated as outlined below (relative error of 12\%). Standard deviations are provided for comparison purposes.

Table S18: Experimental tunneling half-lives of the reaction $\mathbf{1 t} \rightarrow \mathbf{2}$ obtained by evaluating the strongest band at $1249.4(\mathbf{1 t})$ and $1688.5 \mathrm{~cm}^{-1}(\mathbf{2})$, respectively.

\begin{tabular}{ccccc}
\hline Experimental Conditions & $t_{1 / 2}(\mathbf{1 t}) / \mathrm{h}$ & $t_{1 / 2}(\mathbf{2}) / \mathrm{h}$ & Mean $/ \mathrm{h}$ & Standard Deviation $/ \mathrm{h}$ \\
\hline $\mathrm{A}$ & 57.8 & 68.0 & $62.9 \pm 7.5$ & 7.2 \\
$\mathrm{~B}$ & 66.0 & 70.7 & $68.4 \pm 8.2$ & 3.3 \\
$\mathrm{C}$ & 77.0 & 71.5 & $74.3 \pm 8.9$ & 3.9 \\
\hline
\end{tabular}

Table S19: Experimental tunneling half-lives of the reaction $9 \mathrm{t} \rightarrow 9 \mathrm{c}$ obtained by evaluating the strongest band at $1058.6(9 \mathrm{t})$ and $1360.5 \mathrm{~cm}^{-1}(9 \mathrm{c})$, respectively.

\begin{tabular}{ccccc}
\hline Experimental Conditions & $t_{1 / 2}(\mathbf{9 t}) / \mathrm{h}$ & $t_{1 / 2}(\mathbf{9 c}) / \mathrm{h}$ & Mean $/ \mathrm{h}$ & Standard Deviation / $\mathrm{h}$ \\
\hline $\mathrm{A}$ & 8.4 & 9.9 & $9.2 \pm 1.1$ & 1.1 \\
$\mathrm{~B}$ & 8.7 & 10.2 & $9.5 \pm 1.1$ & 1.1 \\
$\mathrm{C}$ & 113.6 & 108.3 & $111.0 \pm 13.3$ & 3.7 \\
\hline
\end{tabular}




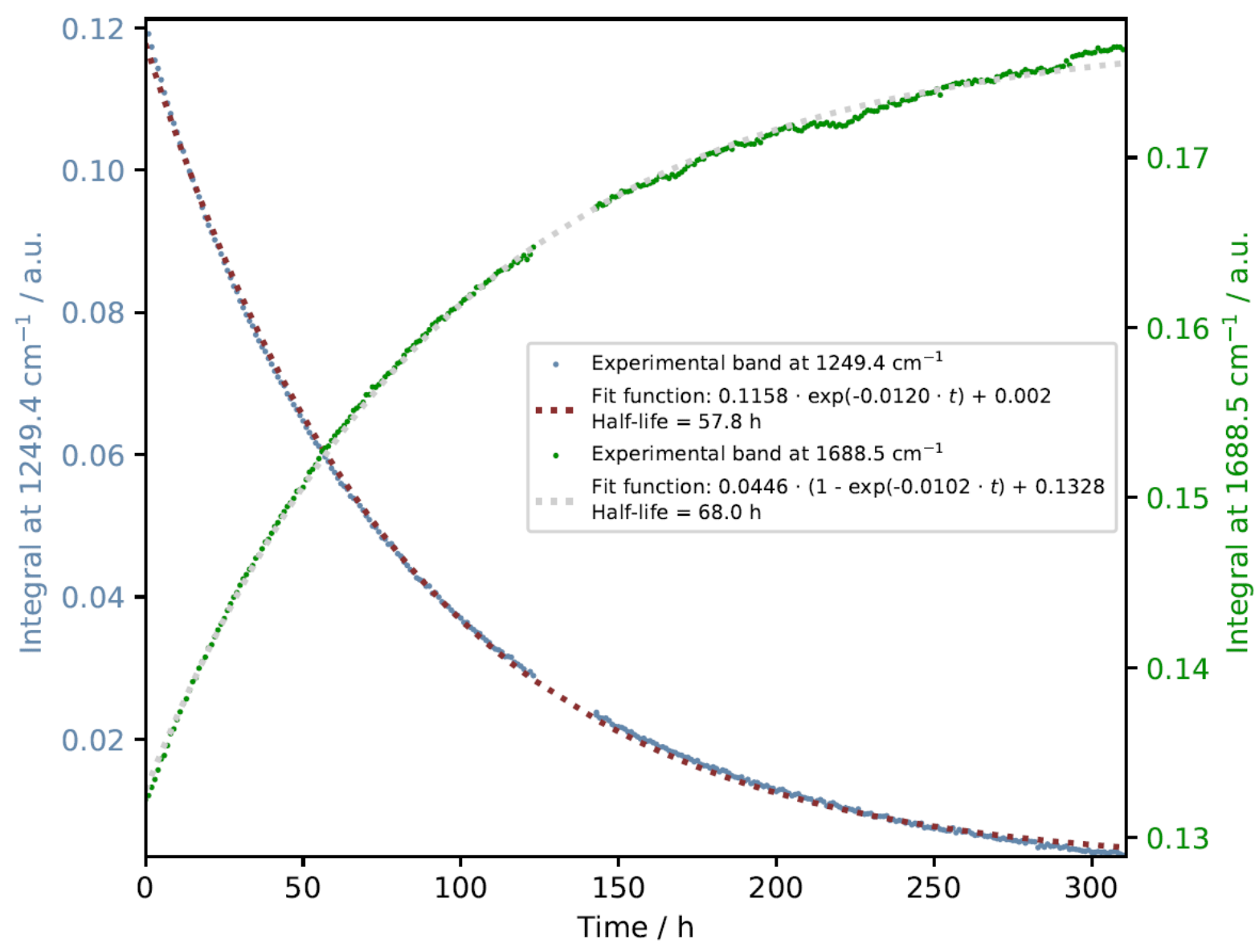

Figure S12: Kinetic evaluation of the reaction $\mathbf{1 t} \rightarrow \mathbf{2}$ at $3 \mathrm{~K}$ without filter (experiment A). 


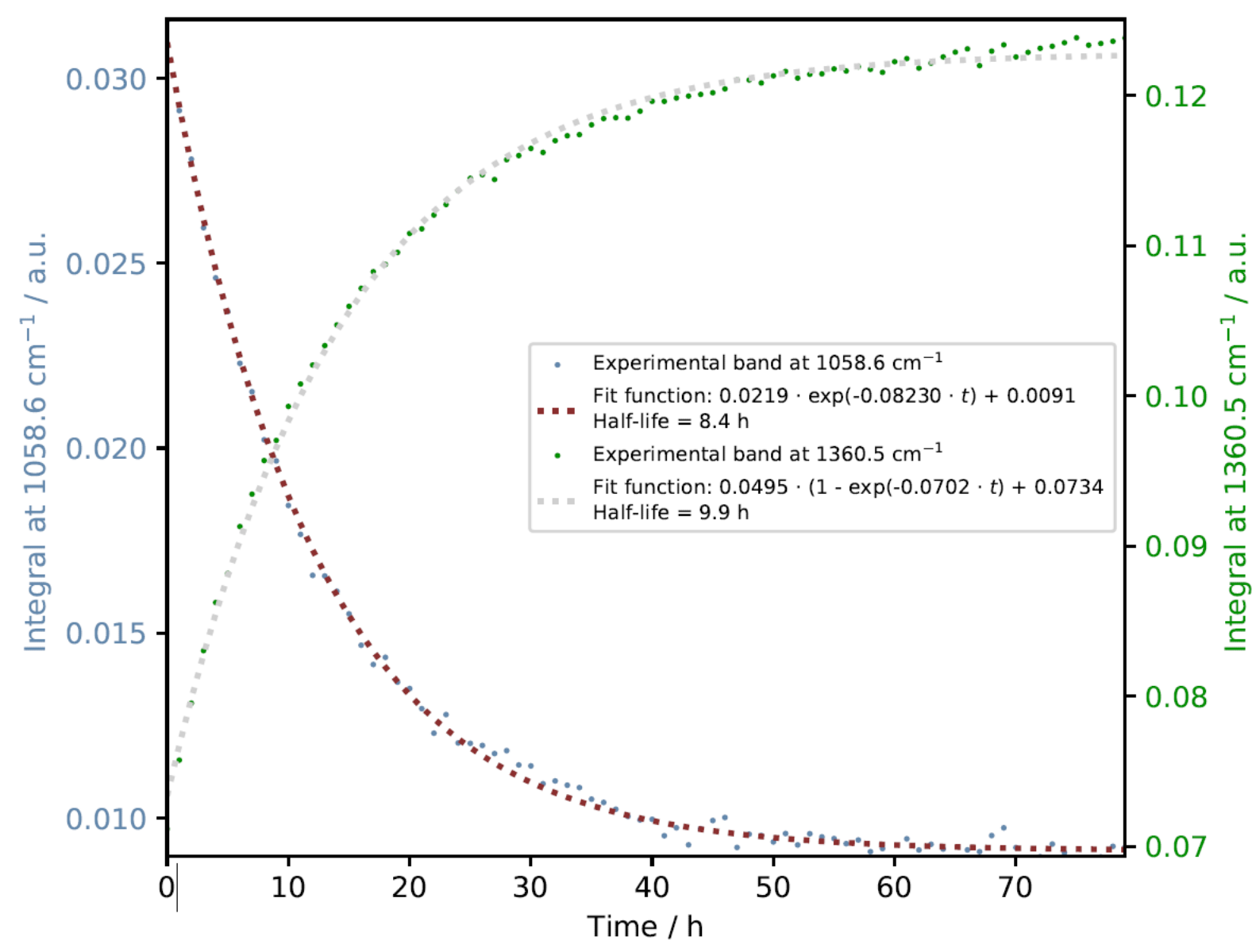

Figure S13: Kinetic evaluation of the reaction $9 \mathbf{t} \rightarrow \mathbf{9 c}$ at $3 \mathrm{~K}$ without filter (experiment $\mathrm{A}$ ). 


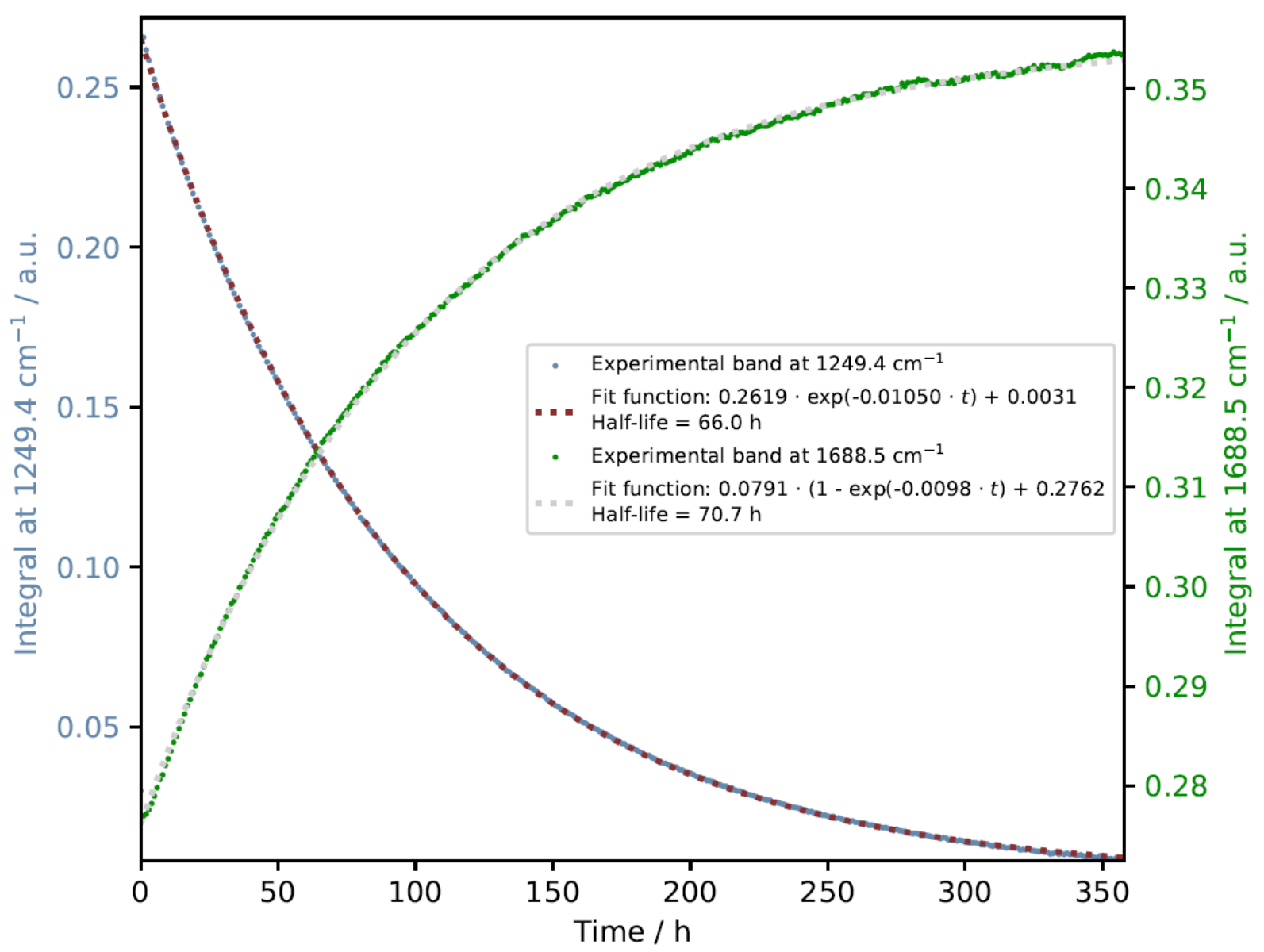

Figure S14: Kinetic evaluation of the reaction $\mathbf{1 t} \rightarrow \mathbf{2}$ at $20 \mathrm{~K}$ without filter (experiment $\mathrm{B}$ ). 


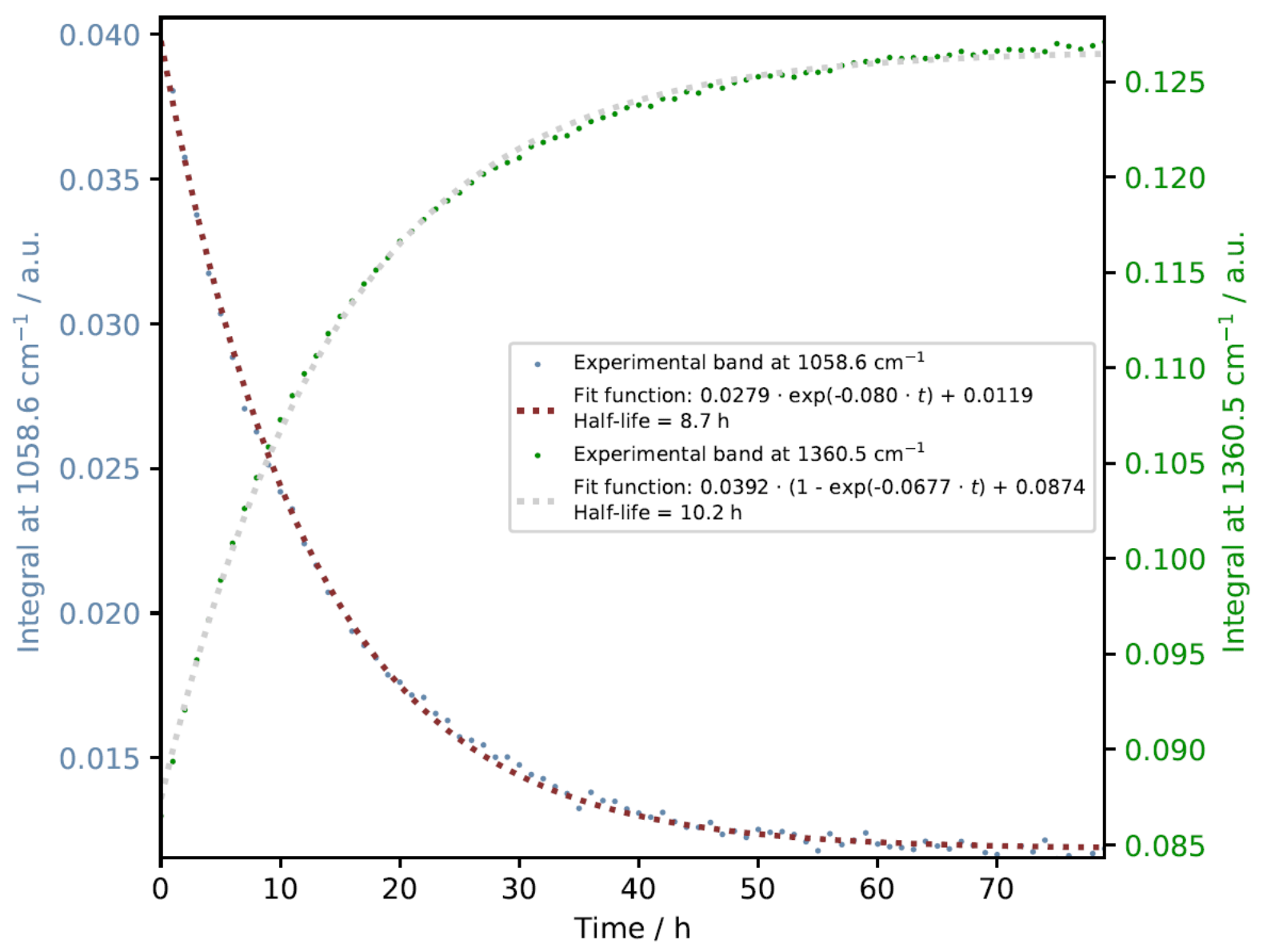

Figure S15: Kinetic evaluation of the reaction $9 t \rightarrow 9 c$ at $20 \mathrm{~K}$ without filter (experiment B). 


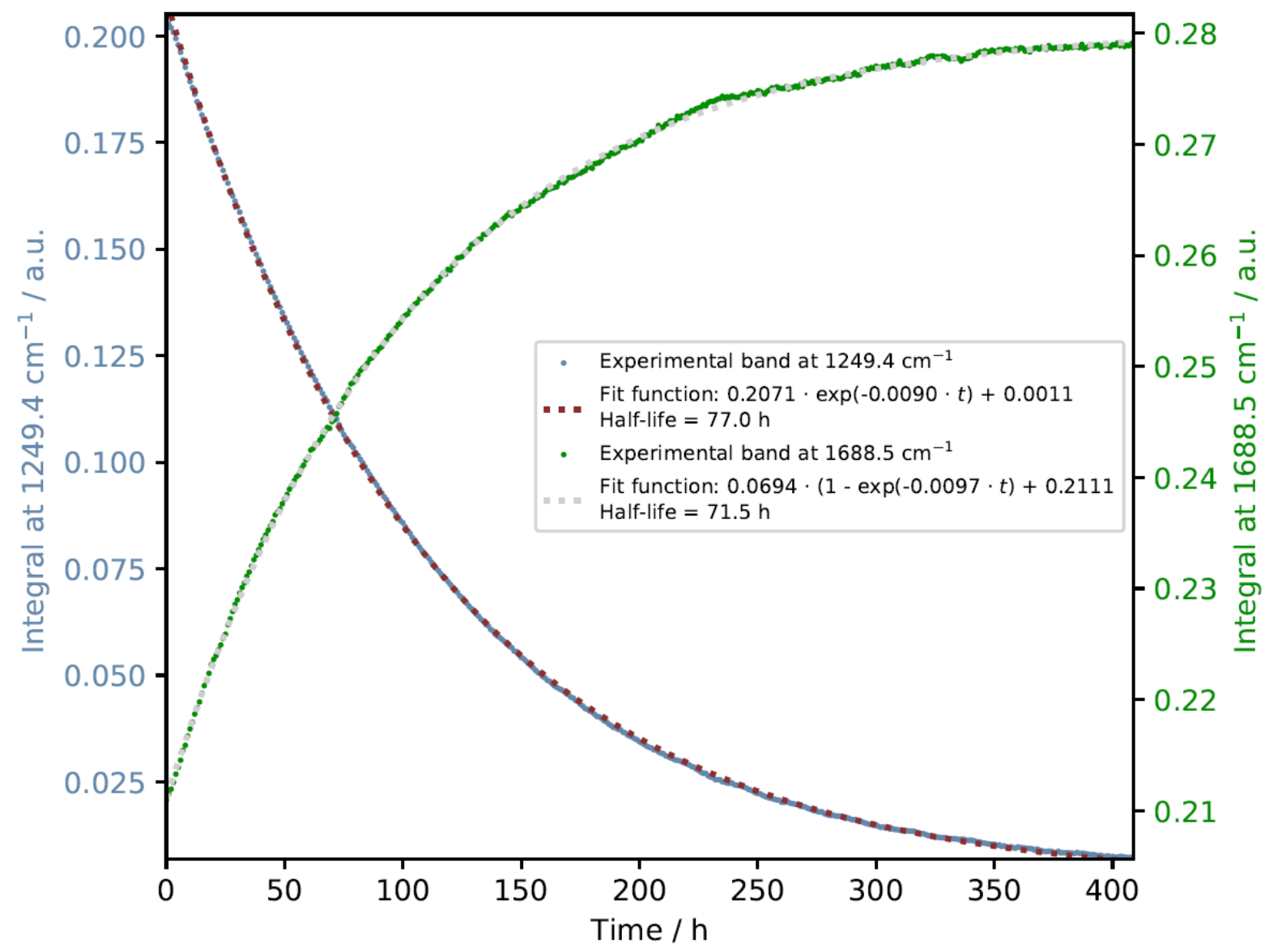

Figure S16: Kinetic evaluation of the reaction $\mathbf{1 t} \rightarrow \mathbf{2}$ at $3 \mathrm{~K}$ with $4.5 \mu \mathrm{m}$ cut-off filter (experiment C). This plot (without details of the fit function) is also depicted in the main text. 


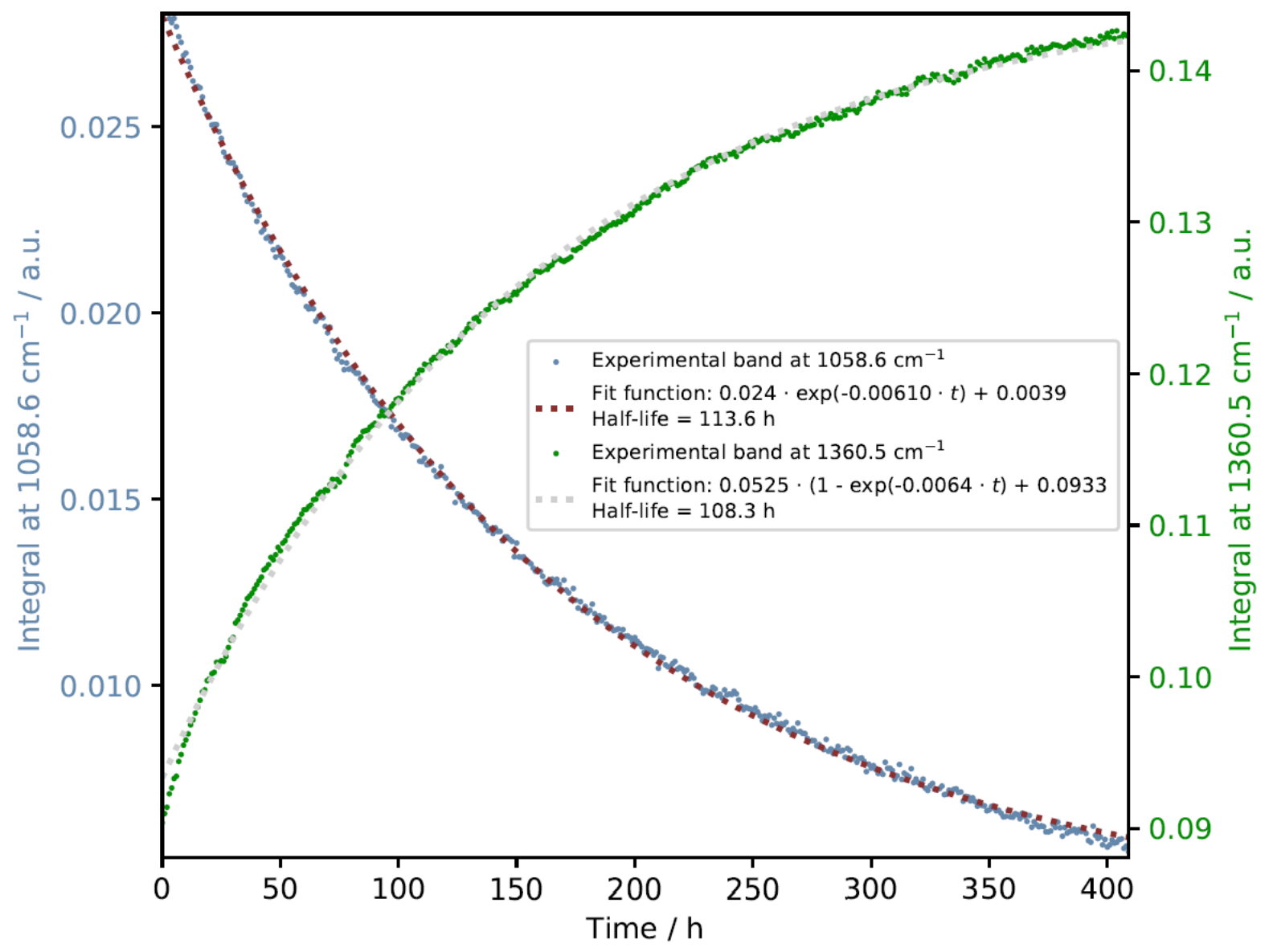

Figure S17: Kinetic evaluation of the reaction $9 t \rightarrow 9 c$ at $3 \mathrm{~K}$ with $4.5 \mu \mathrm{m}$ cut-off filter (experiment $\mathrm{C}$ ).

In contrast to $\mathbf{1 t} \rightarrow \mathbf{2}$, the $\mathbf{9 t} \rightarrow \mathbf{9 c}$ reaction is significantly accelerated when the matrix is exposed to the full range of the spectrometer beam (ca. $9 \mathrm{~h}$ versus $111 \mathrm{~h}$ ). Without using a filter, thermal excitation through the spectrometer is apparently still an important contributor to this reaction even at $3 \mathrm{~K}$. This result can be rationalized on the basis of quantum chemical computations as the associated activation barrier for $9 \mathrm{t} \rightarrow 9 \mathrm{c}$ is only $9.6 \mathrm{kcal} \mathrm{mol}^{-1}$ (vide infra) and can therefore be readily overcome by the energy introduced by the spectrometer beam. When using a cut-off filter, which only lets pass light with $\lambda \geq 4.5 \mu \mathrm{m}\left(E \leq 6.4 \mathrm{kcal} \mathrm{mol}^{-1}\right)$, the reaction presumably is mostly facilitated by QMT. The intrinsic tunneling half-life of $9 t$ is thus presumably more accurately represented with $111 \mathrm{~h}$ rather than $9 \mathrm{~h}$, even though our tunneling computation yields $8.60 \mathrm{~h}$ (vide infra). The perfect agreement between the computed and the experimental value obtained without using the cut-off filter is most likely a coincidence.

In Figure S18 to S34 we present the kinetic evaluation of all assigned bands of $1 \mathrm{t}, \mathbf{2 ,}, \mathbf{9 t}$, and $\mathbf{9 c}$ at $20 \mathrm{~K}$ without using the cut-off filter (experiment $B$ ). We decided to perform this thorough analysis with the data obtained from experiment $B$, because all bands are visible, which is, of course, not the case in the experiment performed with the filter (experiment $\mathrm{C}$ ). At $3 \mathrm{~K}$ and without the filter, some data points are missing as mentioned above (experiment A). The results are listed in Table S20. The errors of the mean values were obtained from the largest deviation of the half-life data from the mean. For both reactions the relative error amounts to ca. $12 \%$. We assume the relative error to be in the same range in the other two experiments $\mathrm{A}$ and $\mathrm{C}$ at $3 \mathrm{~K}$ (with and without the cut-off filter). Apparently, the halflife of the decaying species (1t, and $\mathbf{9 t})$ is systematically lower than for the increasing species $(\mathbf{2}$, and 
9c). This is presumably mostly due to a shift of the baseline towards higher intensities throughout one experiment. This shift is unavoidable in kinetic measurements carried out for several weeks as is the influx of small amounts of external water and $\mathrm{CO}_{2}$ into the system. For this reason, rather large errors are expected. We are, however, confident that we obtained reasonably accurate ranges for $t_{1 / 2}$ of both reactions.

Table S20: Thorough kinetic analysis of all assigned bands that increase (decrease). The values were obtained from the measurement at $20 \mathrm{~K}$ without the cut-off filter (experiment B). Means for each species and each reaction are provided.

\begin{tabular}{|c|c|c|c|}
\hline Species & Band $/ \mathrm{cm}^{-1}$ & Tunneling half-life / $\mathrm{h}$ & \multirow{9}{*}{$63.1 \pm 2.9$} \\
\hline \multirow{8}{*}{$1 \mathrm{t}$} & & & \\
\hline & 797.7 & 63.0 & \\
\hline & 891.5 & 63.0 & \\
\hline & 1249.4 & 66.0 & \\
\hline & 1327.1 & 61.3 & \\
\hline & 2036.2 & 64.8 & \\
\hline & 3306.9 & 61.3 & \\
\hline & 3545.0 & 64.8 & \\
\hline \multirow{5}{*}{2} & 648.2 & 75.3 & \\
\hline & 940.1 & 76.2 & \\
\hline & 1688.5 & 70.7 & $74.1 \pm 3.4$ \\
\hline & 2107.5 & 75.3 & \\
\hline & 2868.0 & 73.0 & \\
\hline \multirow{3}{*}{$9 t$} & 1058.6 & 8.7 & \multirow{8}{*}{$9.6 \pm 1.1$} \\
\hline & 1770.0 & 9.6 & \\
\hline & 3558.4 & 8.8 & \\
\hline \multirow{5}{*}{ 9c } & 1081.9 & 8.9 & \\
\hline & 1193.0 & 10.0 & \\
\hline & 1360.5 & 10.2 & \\
\hline & 1804.0 & 10.7 & \\
\hline & 3450.0 & 10.2 & \\
\hline
\end{tabular}




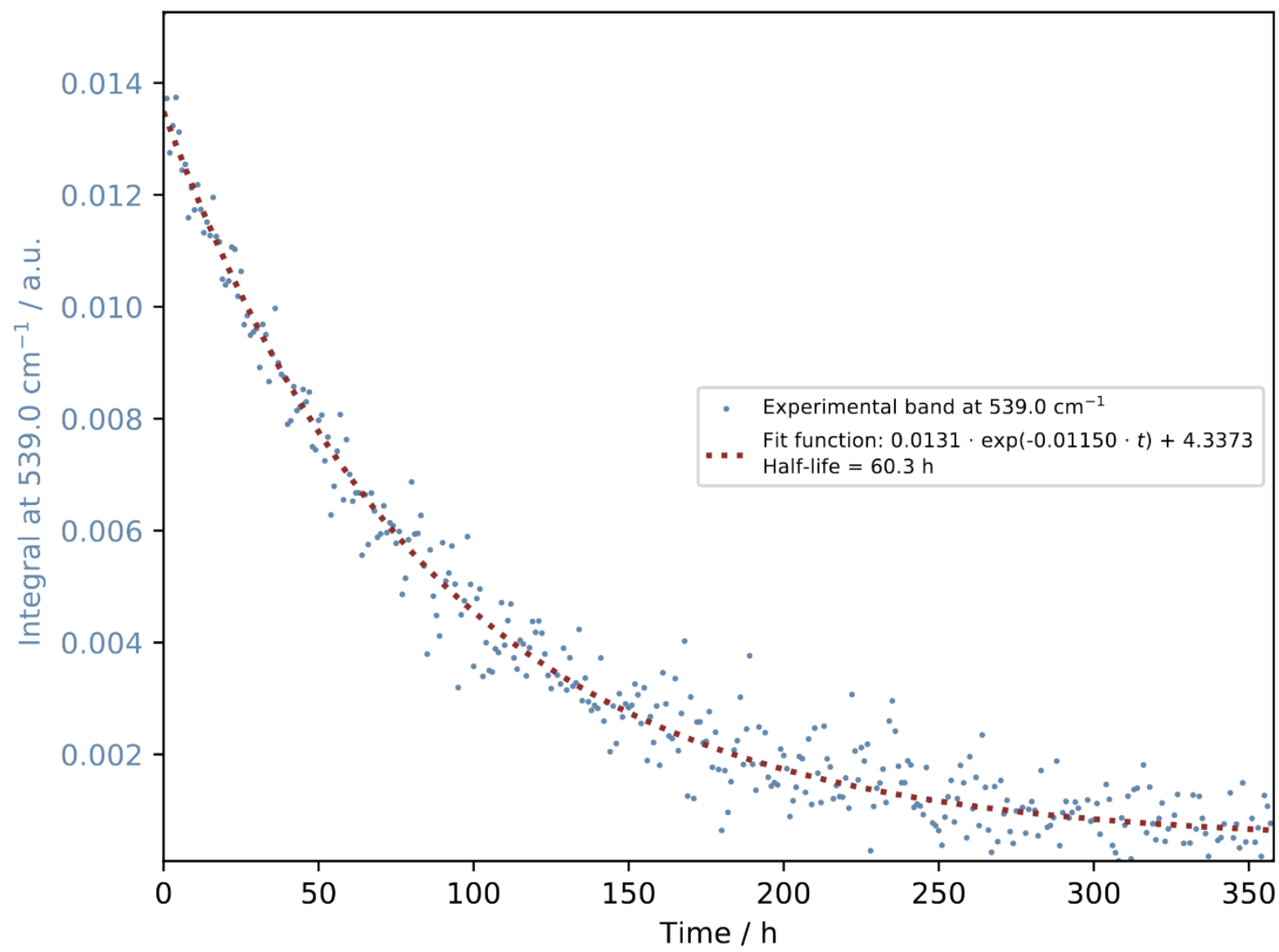

Figure S18: Kinetic analysis of $\mathbf{1 t}$ at $20 \mathrm{~K}$ without filter (experiment B). 


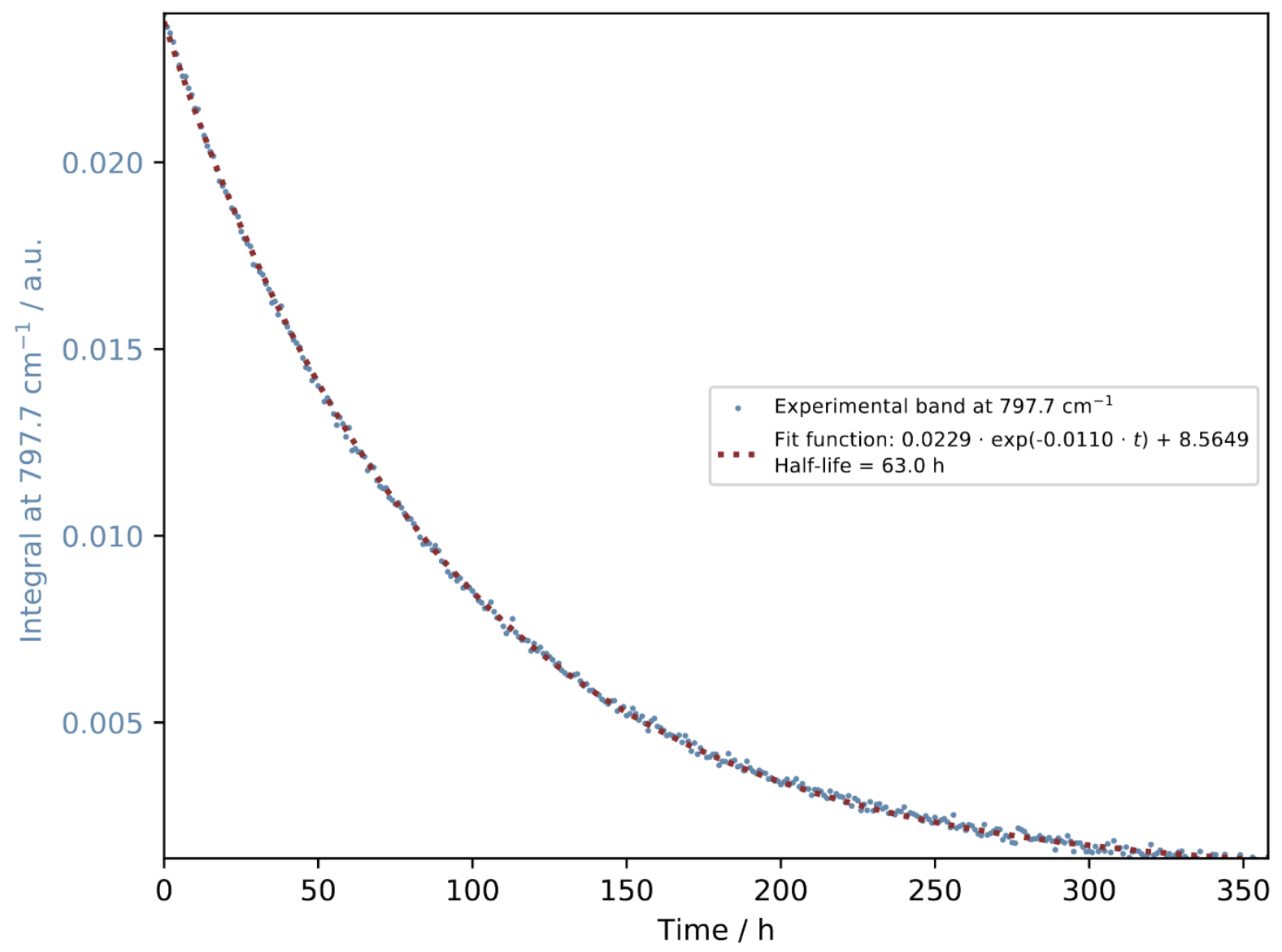

Figure S19: Kinetic analysis of $1 \mathbf{t}$ at $20 \mathrm{~K}$ without filter (experiment B). 


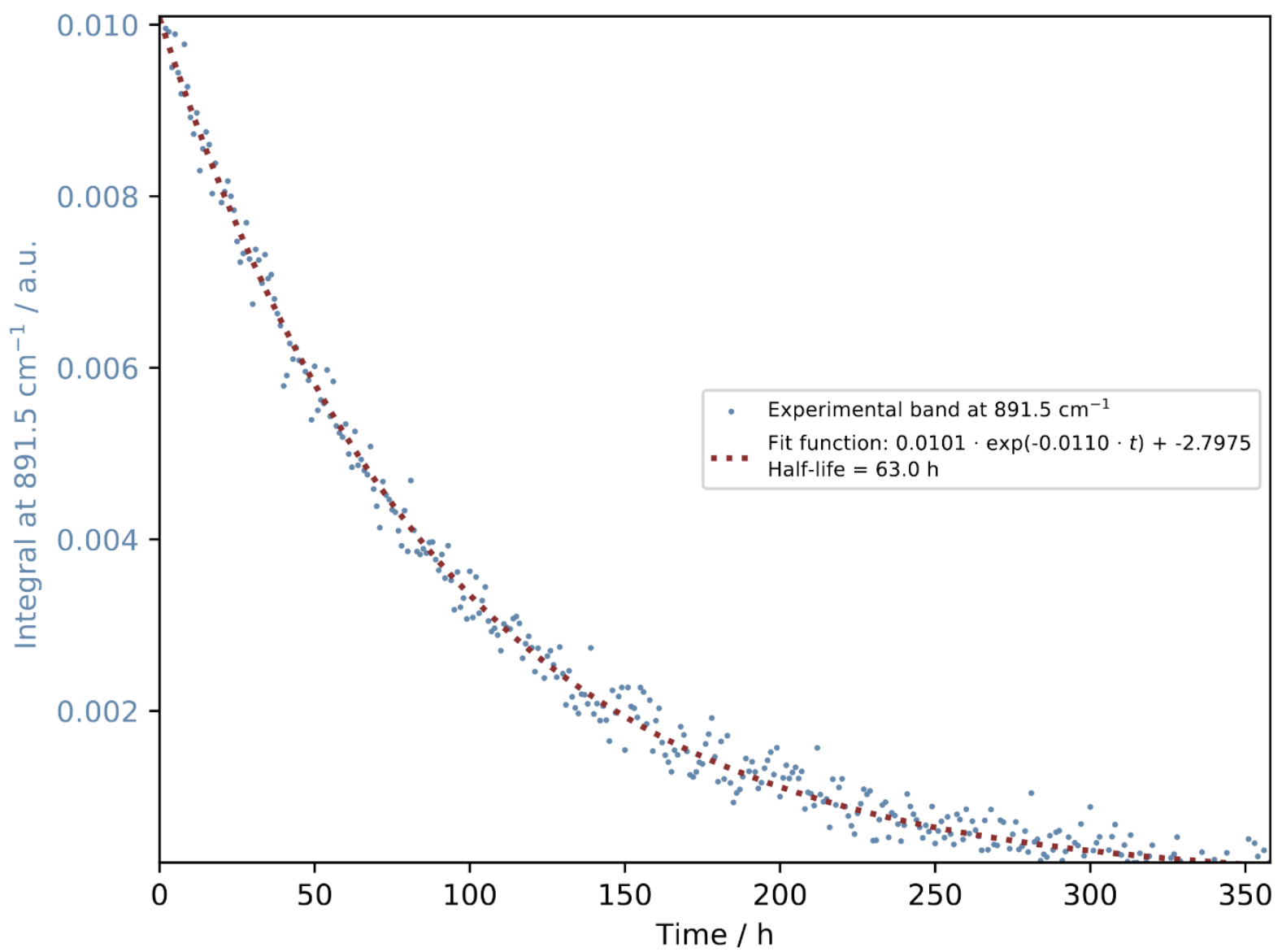

Figure S20: Kinetic analysis of $\mathbf{1 t}$ at $20 \mathrm{~K}$ without filter (experiment B). 


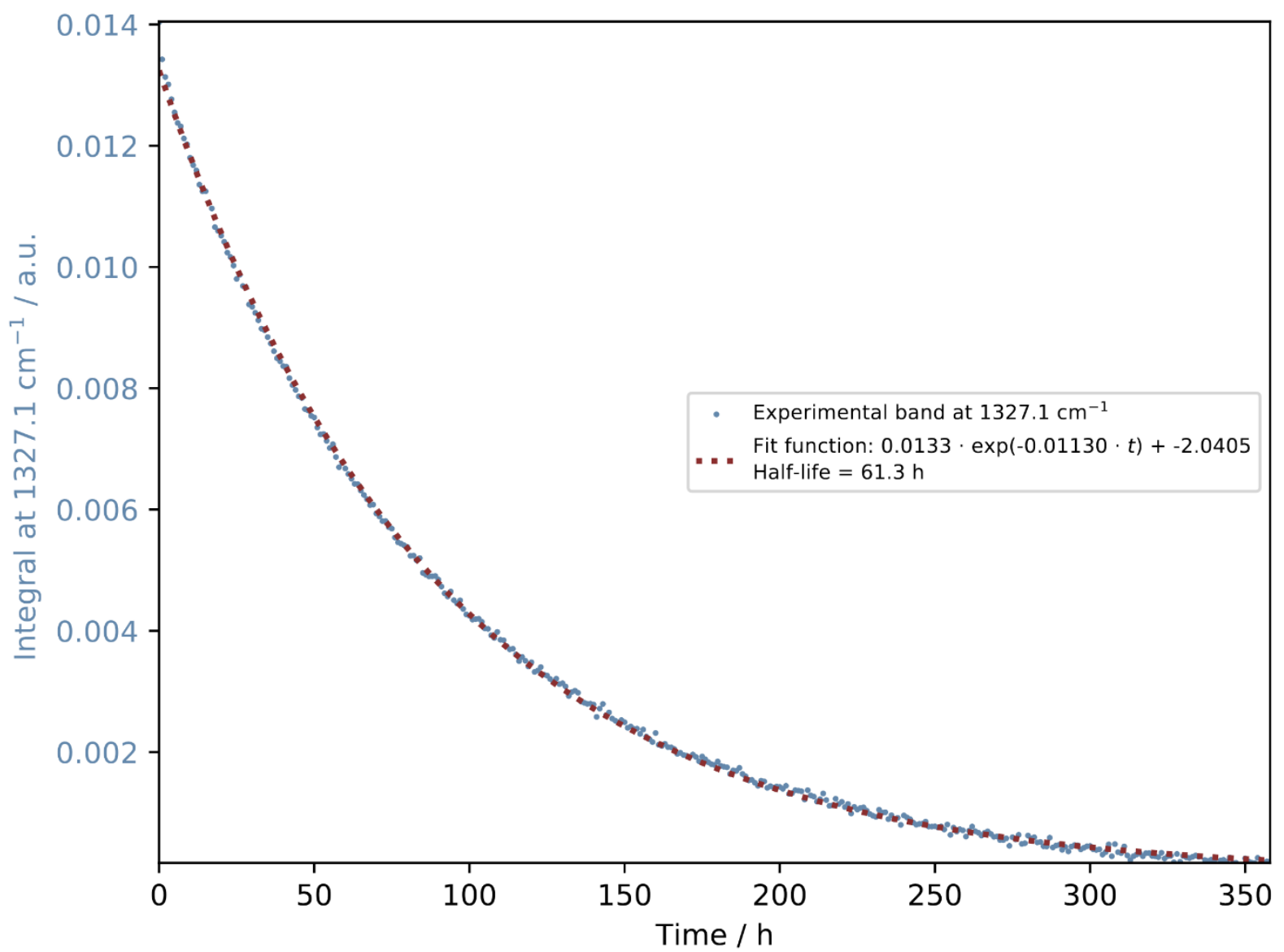

Figure S21: Kinetic analysis of $1 \mathrm{t}$ at $20 \mathrm{~K}$ without filter (experiment B). 


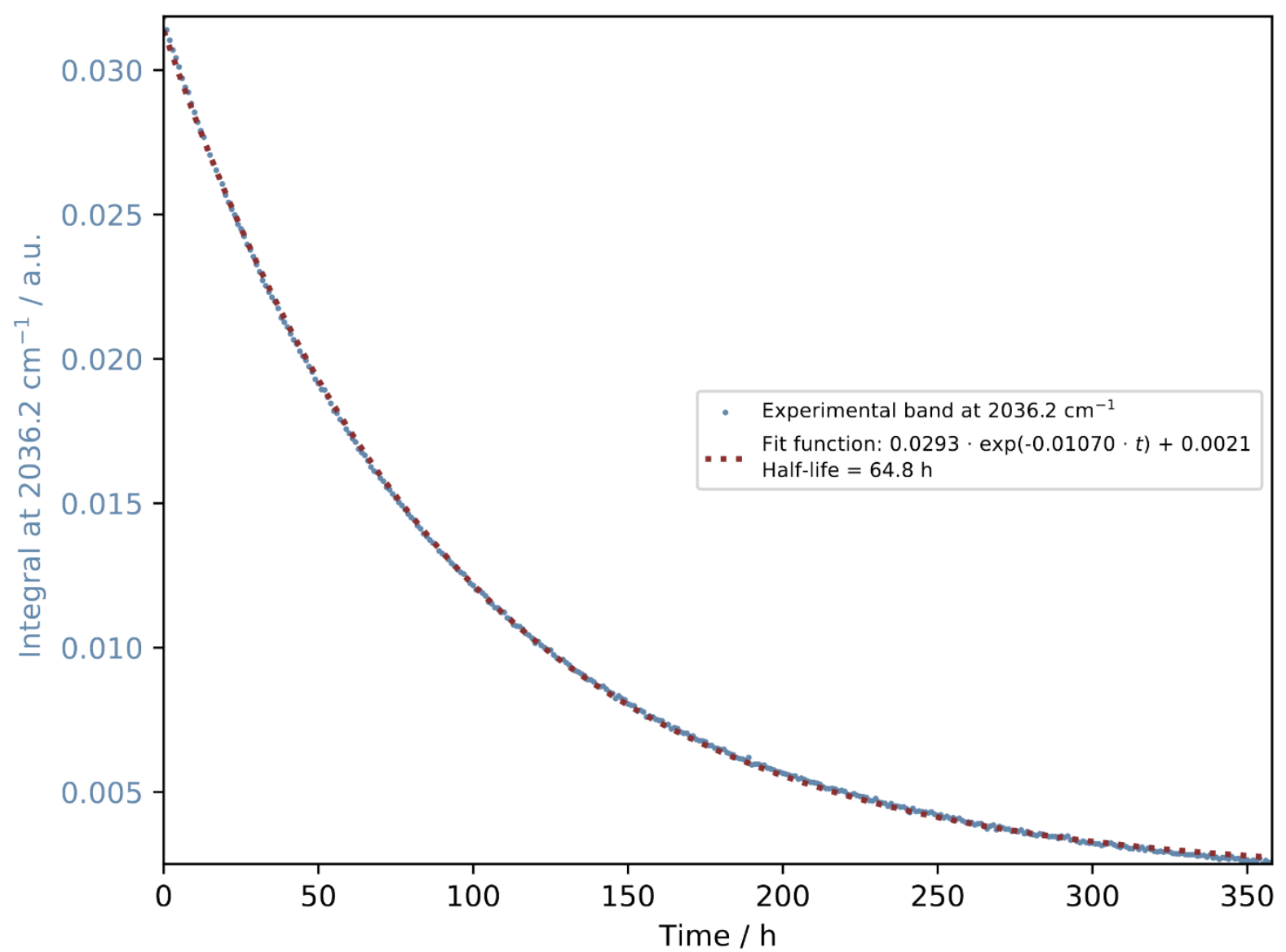

Figure S22: Kinetic analysis of $1 \mathrm{t}$ at $20 \mathrm{~K}$ without filter (experiment B). 


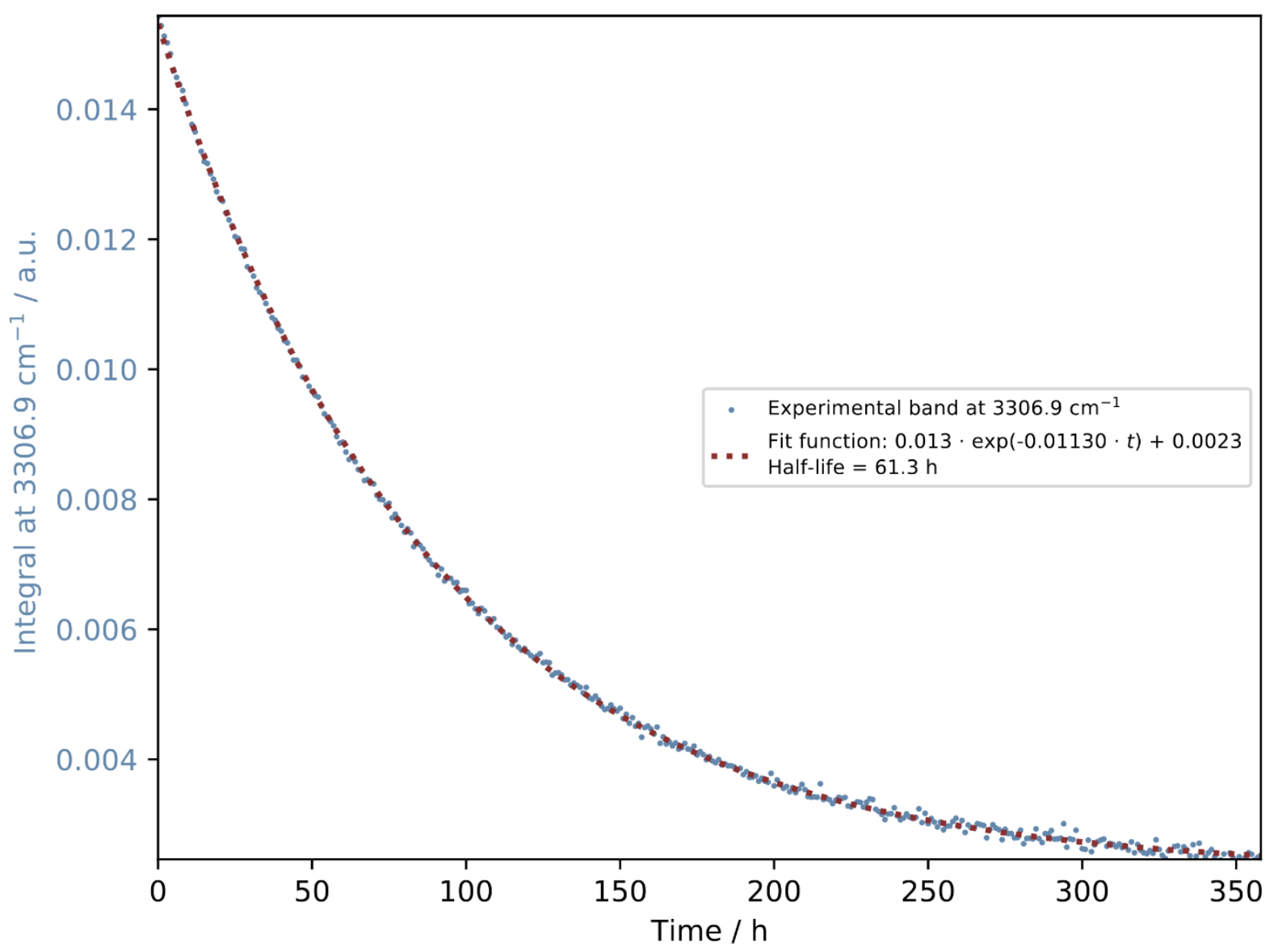

Figure S23: Kinetic analysis of $\mathbf{1 t}$ at $20 \mathrm{~K}$ without filter (experiment $\mathrm{B}$ ). 


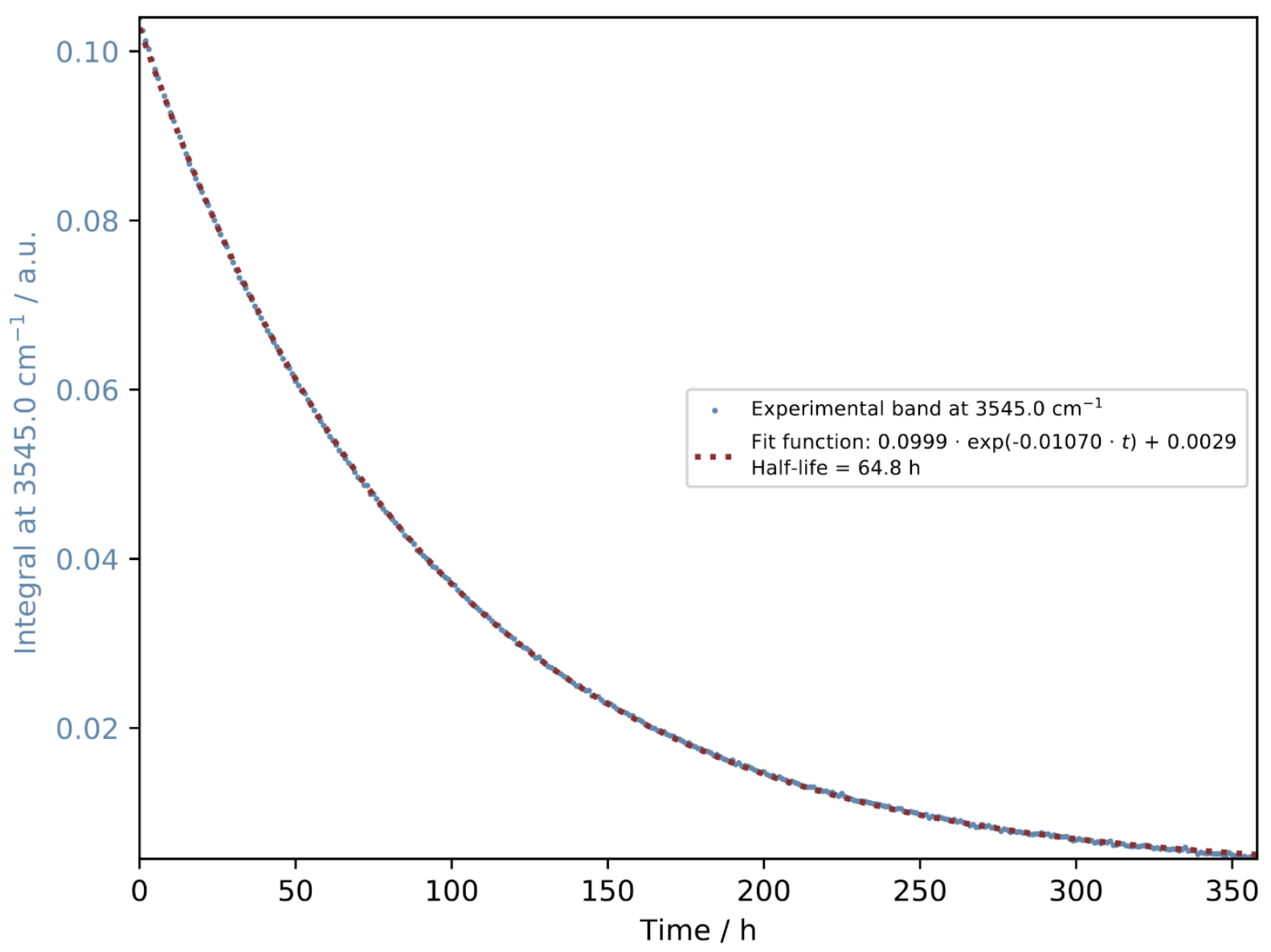

Figure S24: Kinetic analysis of $1 \mathrm{t}$ at $20 \mathrm{~K}$ without filter (experiment $\mathrm{B}$ ). 


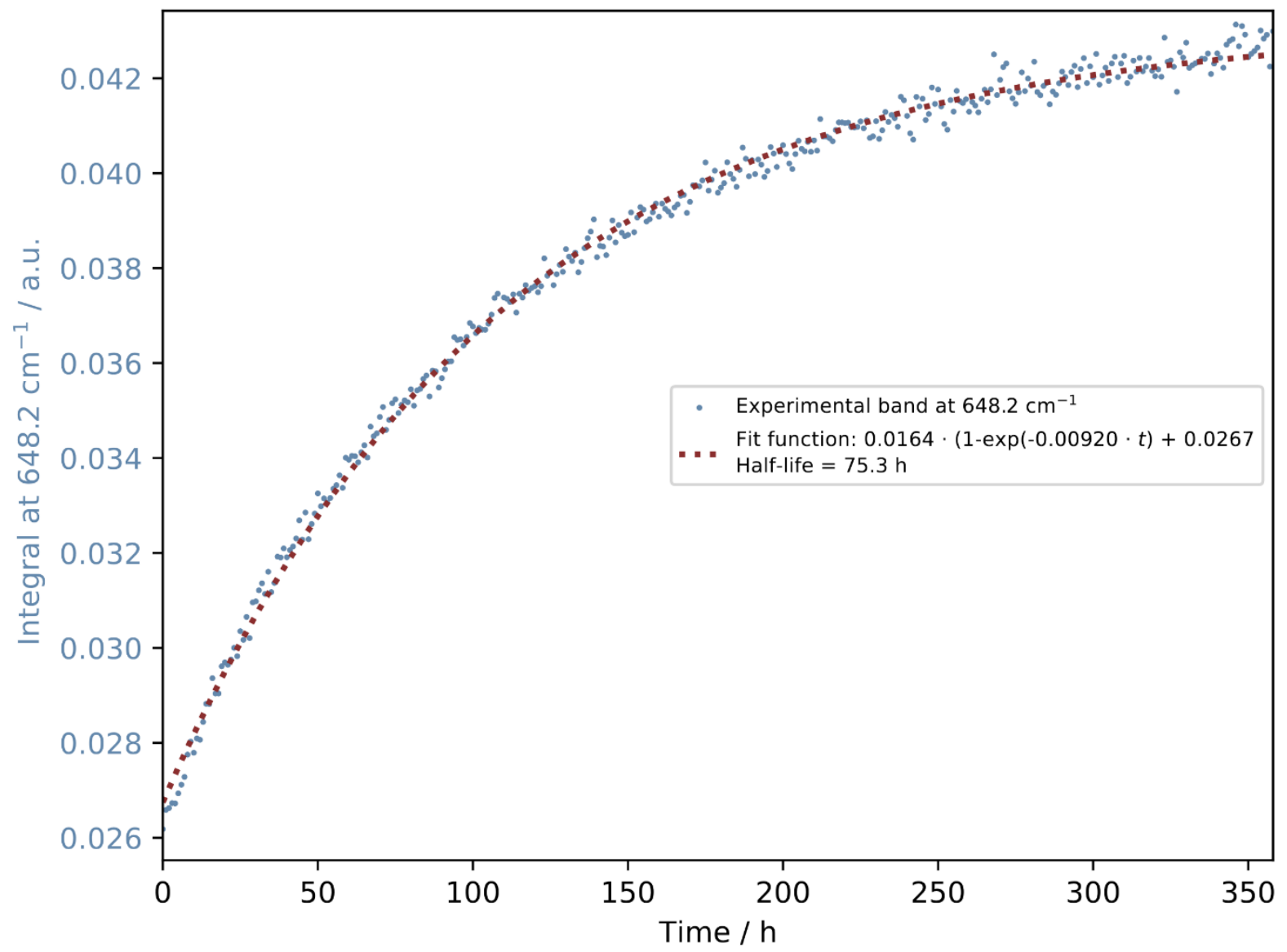

Figure S25: Kinetic analysis of $\mathbf{2}$ at $20 \mathrm{~K}$ without filter (experiment B). 


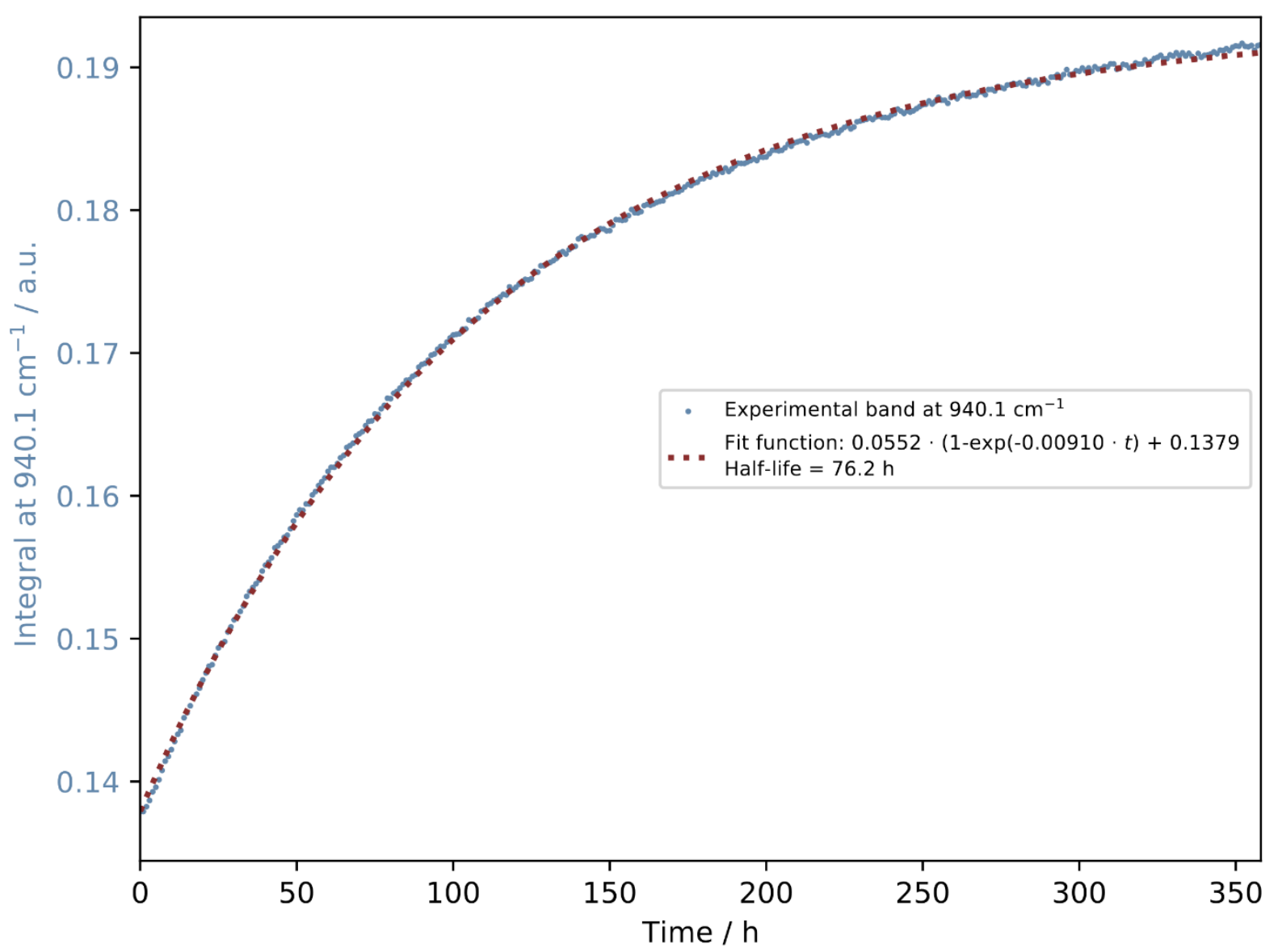

Figure S26: Kinetic analysis of $\mathbf{2}$ at $20 \mathrm{~K}$ without filter (experiment B). 


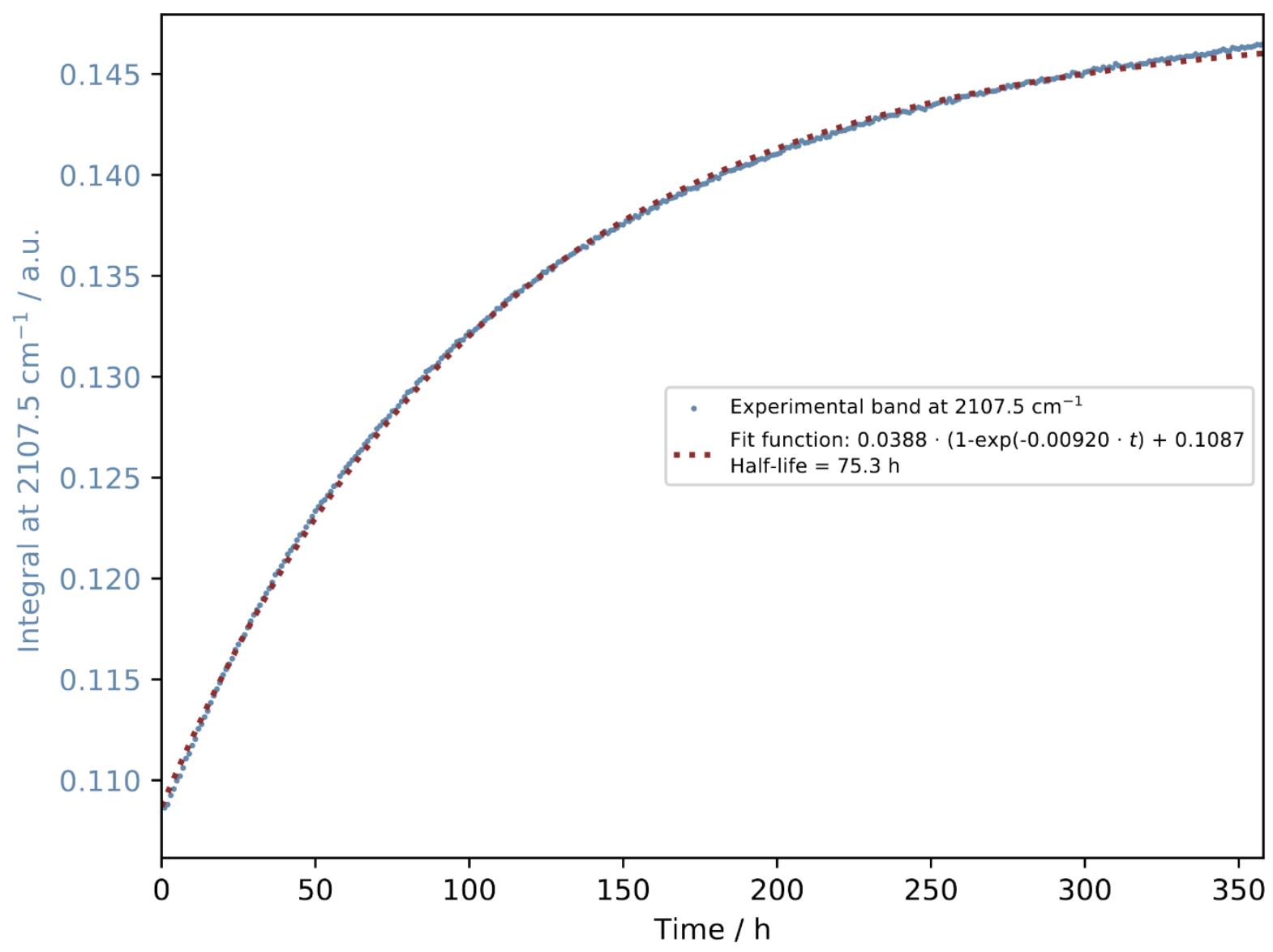

Figure S27: Kinetic analysis of $\mathbf{2}$ at $20 \mathrm{~K}$ without filter (experiment B). 


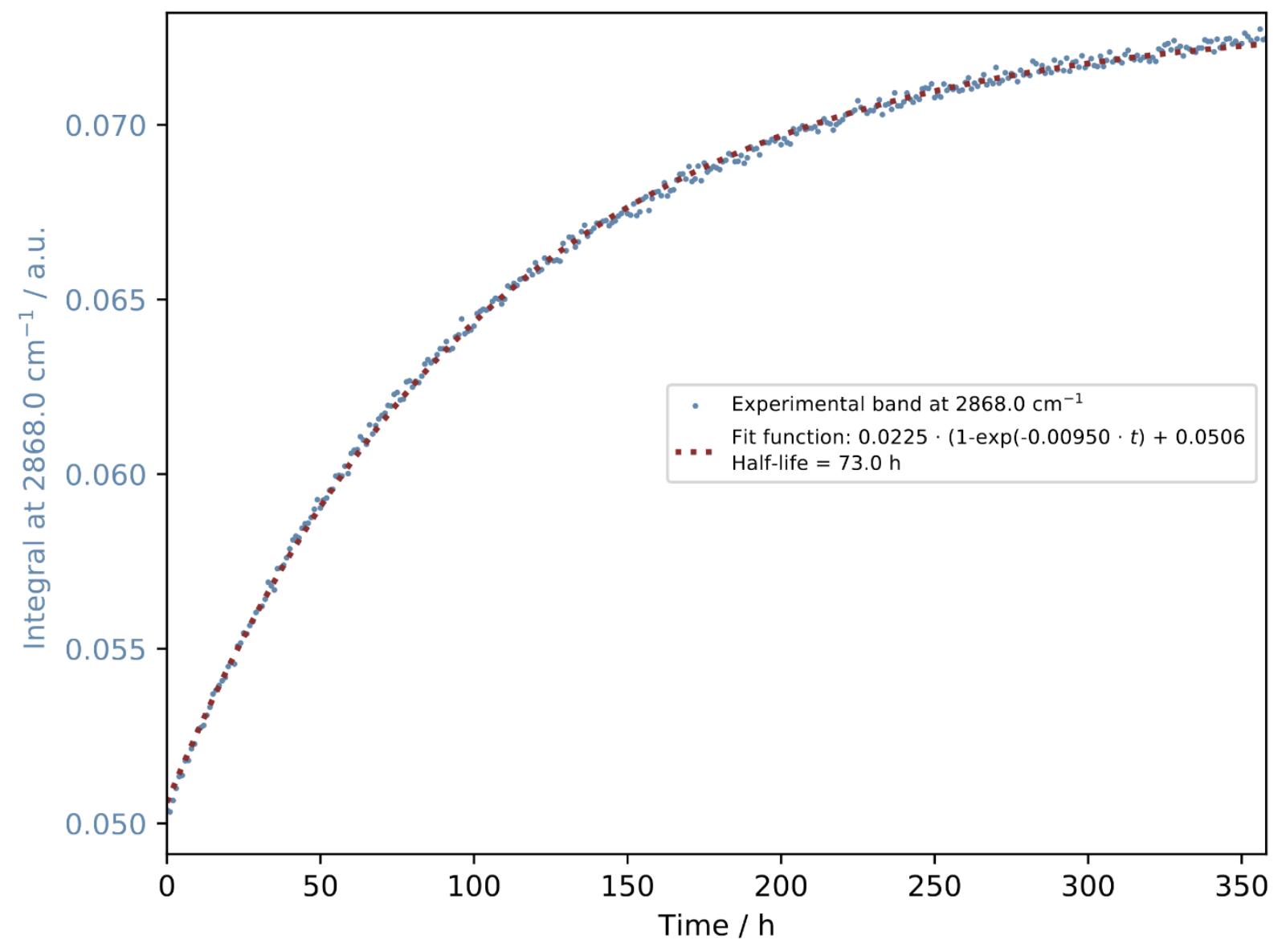

Figure S28: Kinetic analysis of $\mathbf{2}$ at $20 \mathrm{~K}$ without filter (experiment B). 


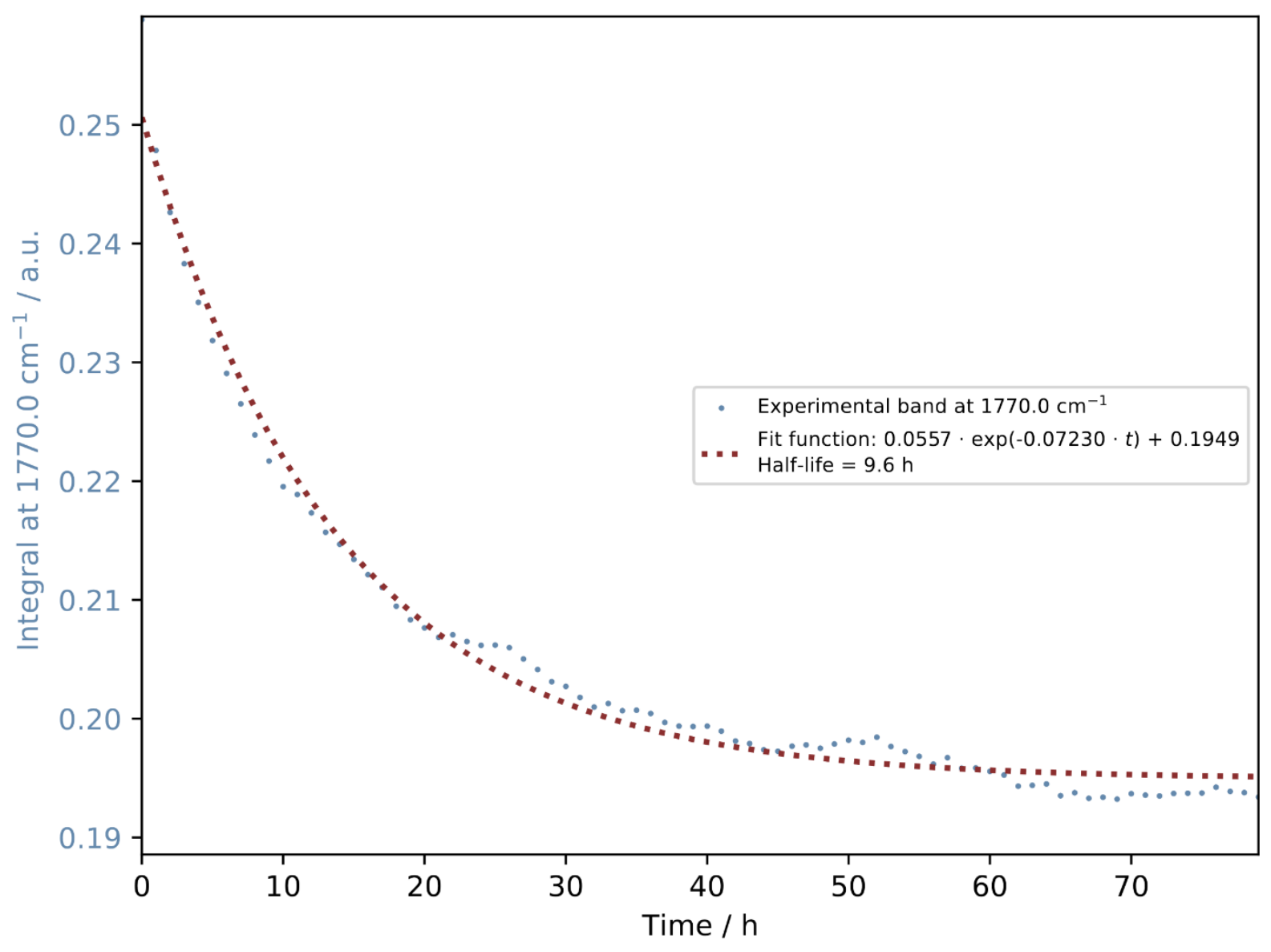

Figure S29: Kinetic analysis of $9 \mathrm{t}$ at $20 \mathrm{~K}$ without filter (experiment B). 


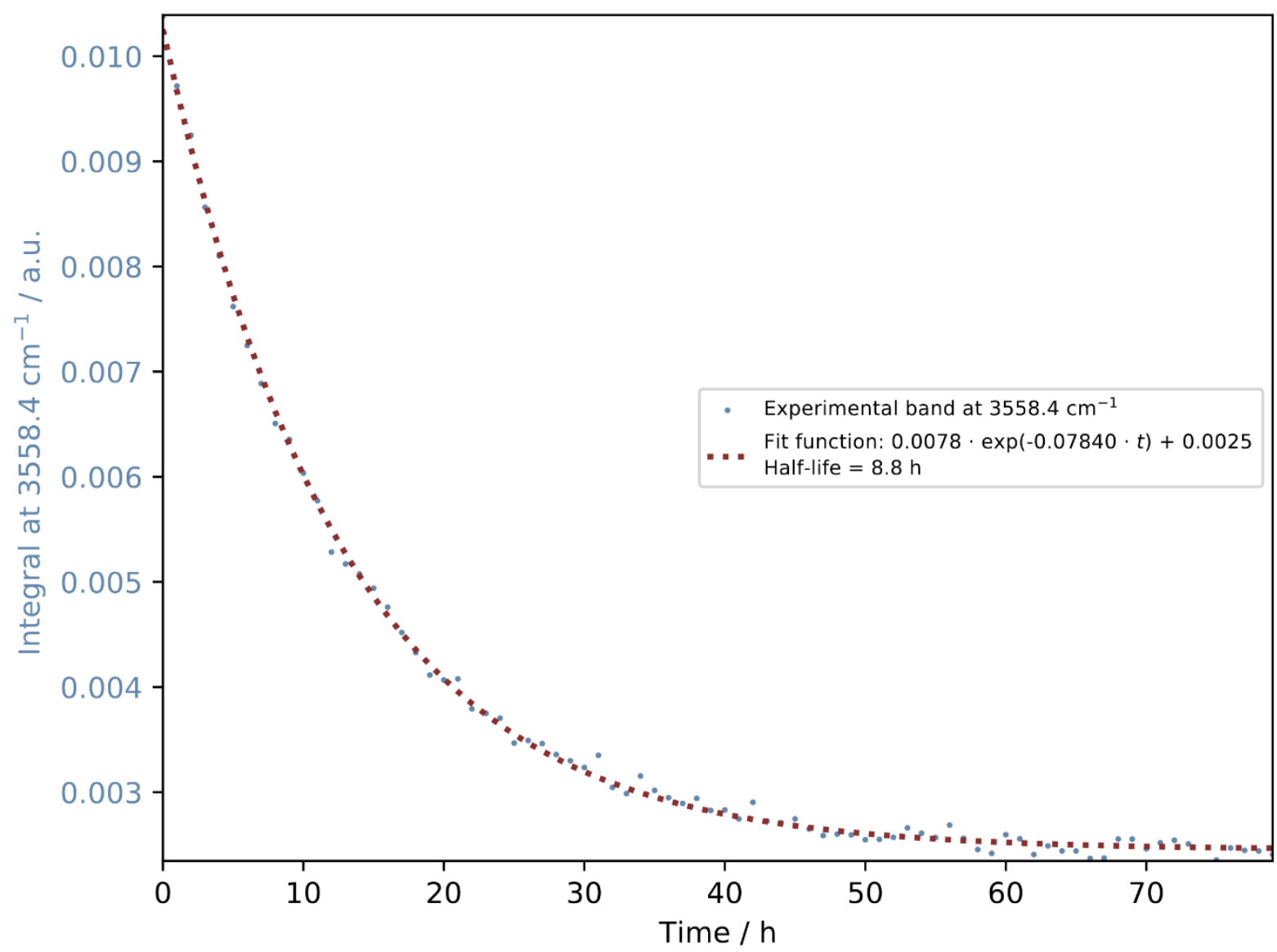

Figure S30: Kinetic analysis of $9 \mathrm{t}$ at $20 \mathrm{~K}$ without filter (experiment B). 


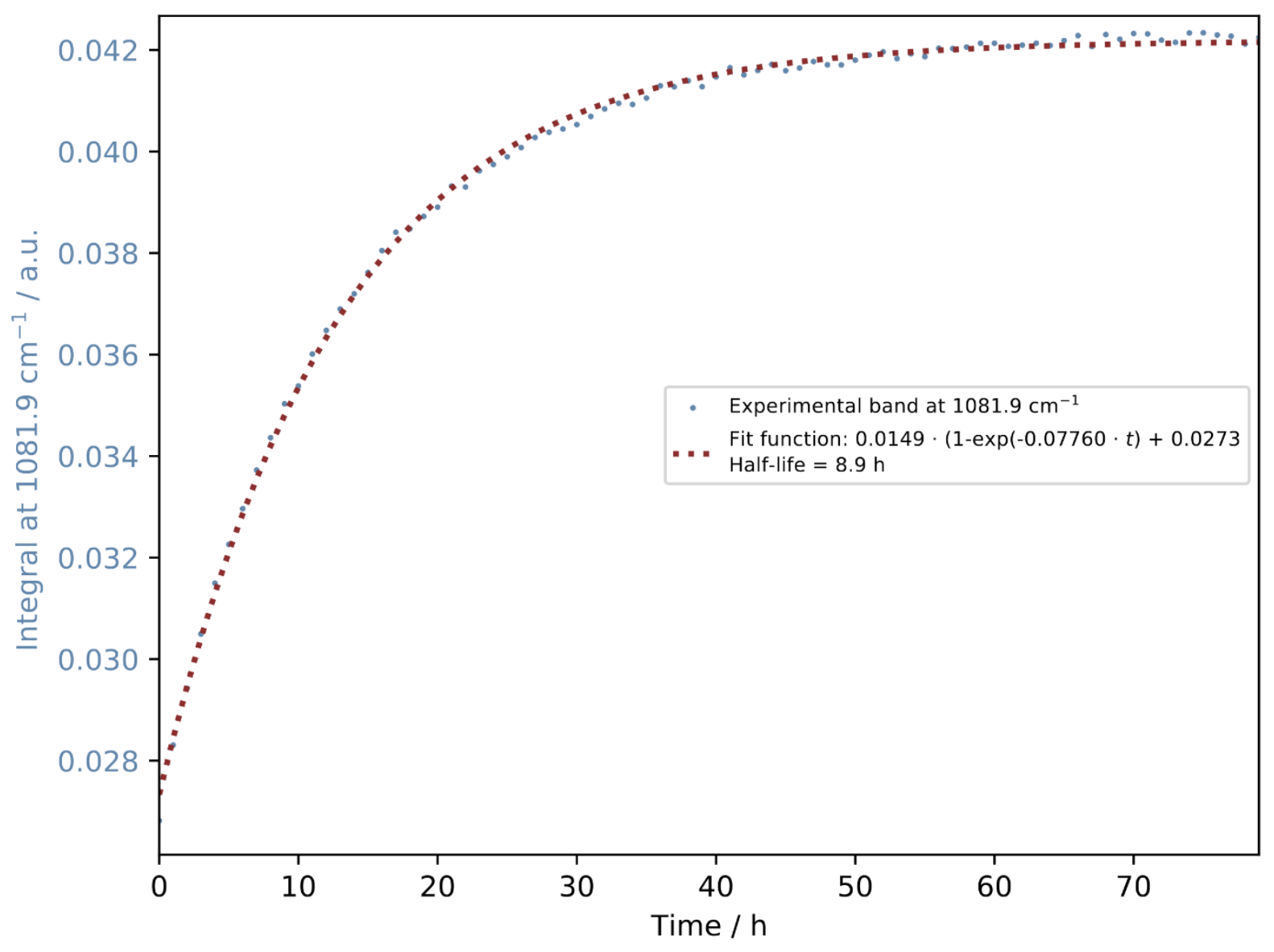

Figure S31: Kinetic analysis of $9 \mathrm{c}$ at $20 \mathrm{~K}$ without filter (experiment $\mathrm{B}$ ). 


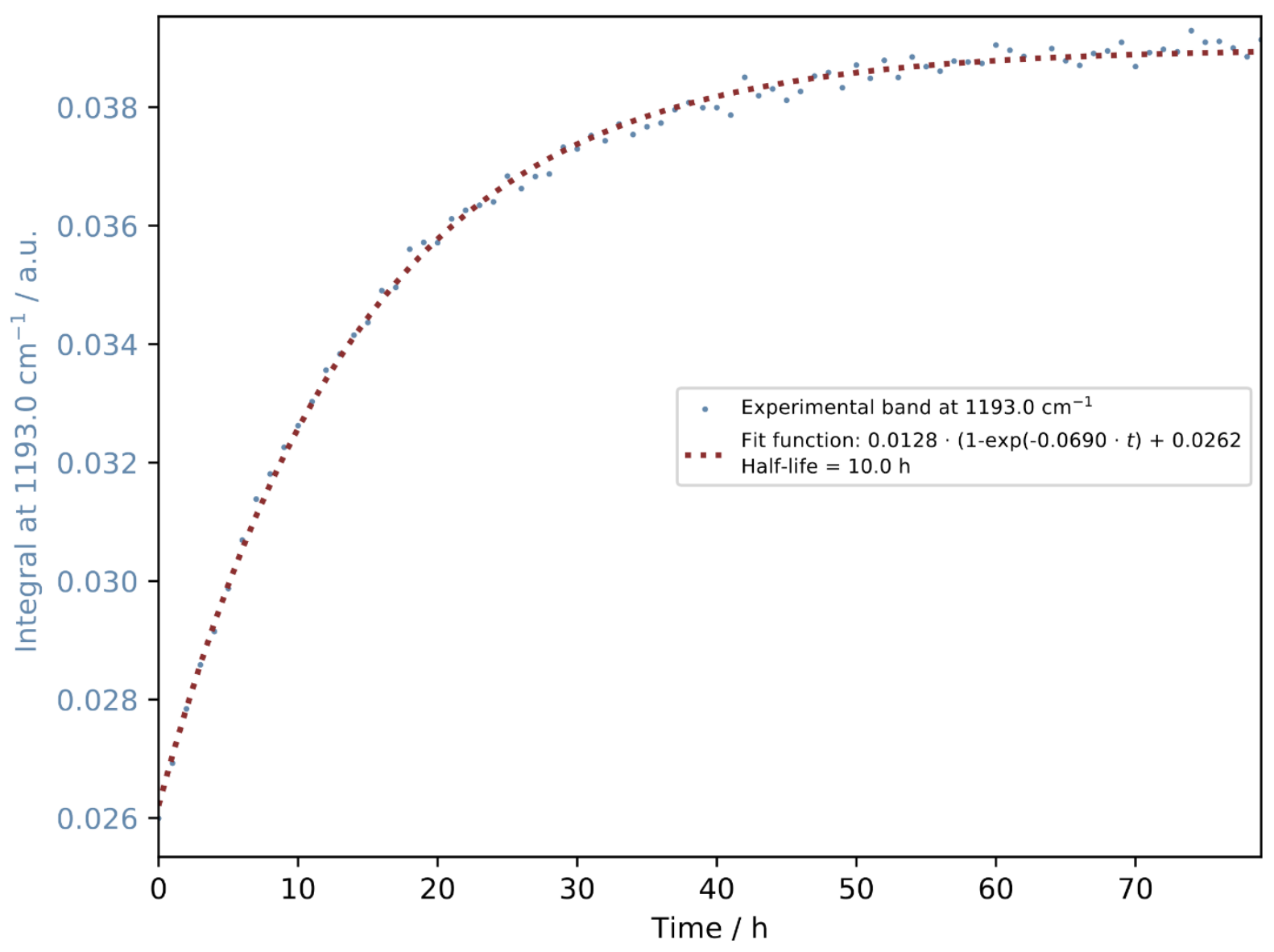

Figure S32: Kinetic analysis of $9 \mathrm{c}$ at $20 \mathrm{~K}$ without filter (experiment B). 


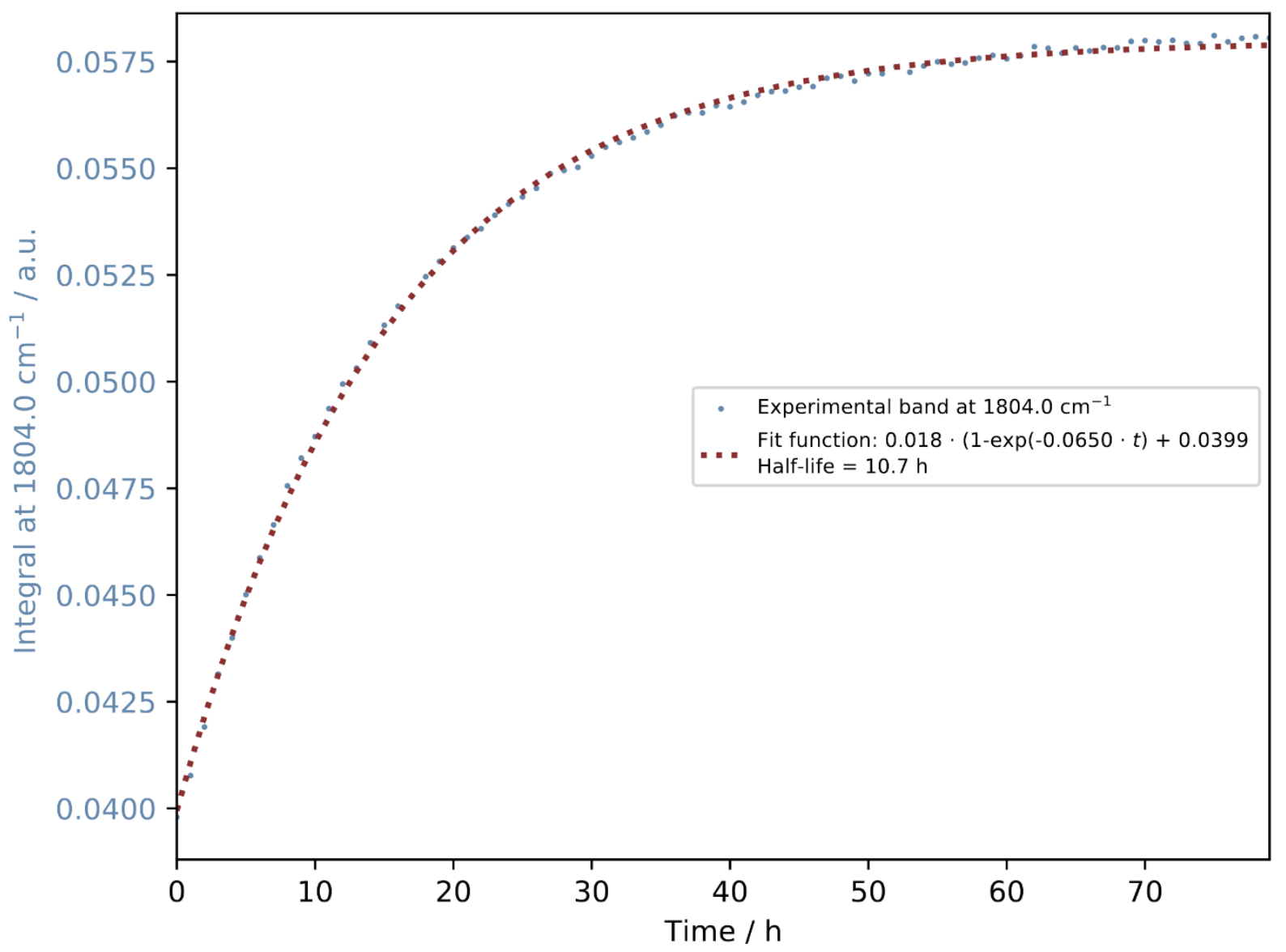

Figure S33: Kinetic analysis of $9 \mathrm{c}$ at $20 \mathrm{~K}$ without filter (experiment B). 


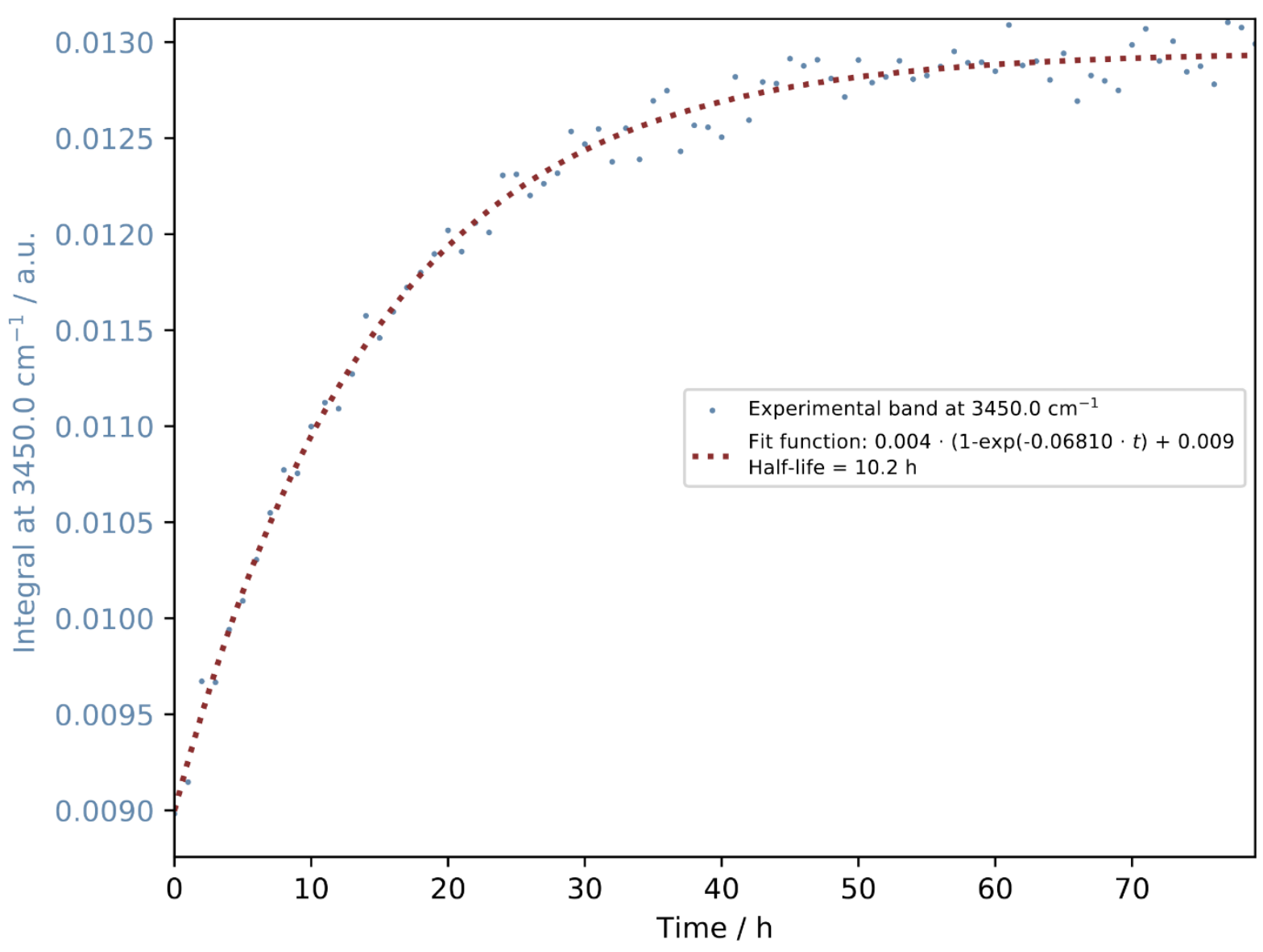

Figure S34: Kinetic analysis of $9 \mathrm{c}$ at $20 \mathrm{~K}$ without filter (experiment $\mathrm{B}$ ). 


\section{Computations}

All DFT computations were performed with the Gaussian 16, Revision C.01 program package (full citations for electronic structure codes are given at the end of this document) at the B3LYP ${ }^{6,7} /$ def2QZVPP ${ }^{8,9}$ level of theory. The keywords Opt=(VTight,MaxCyc=50), Freq=NoRaman, and Int=UltraFine were used for the characterization of minima on the PES. For transition structures the keyword Opt $=(T S$,CalcAll,NoEigen,VTight,MaxCyc=50) was used. IRC computations were conducted with IRC $=($ CalcAll,Forward (or Reverse),MaxPoints $=1000$, MaxCycle $=100$,StepSize $=V$, phase $=(W, X, Y, Z)$ ). The parameters $\mathrm{V}-\mathrm{Z}$ had to be adjusted for every computation. UV/Vis absorptions were computed by using the keyword TD=(Singlets, Root=1,NStates=6).

Coupled-cluster computations performed with CFOUR were carried out at the CCSD(T) ${ }^{10,11} /$ cc-pVTZ $^{12-}$ 15 level of theory and the following keywords: FROZEN_CORE=1, CONVERGENCE $=8$, $A B C D T Y P E=A O B A S I S, C C$ PROG $=E C C, S C F+C O N V=8$, and $C C$ CONV=10. Harmonic frequencies were computed analytically with $\mathrm{VIB}=\mathrm{EXACT}$; for transition structures the keyword METHOD=TS was used additionally.

Computational tunneling half-lives were determined at the CVT/SCT//B3LYP/def2-QZVPP level of theory using Polyrate Version 2017-C.

Natural bond orbitals (NBO) were computed with NBO7 at the B3LYP/def2-QZVPP level of theory based on geometries at the same level. ${ }^{16}$ 


\section{Computed Potential Energy Surfaces}

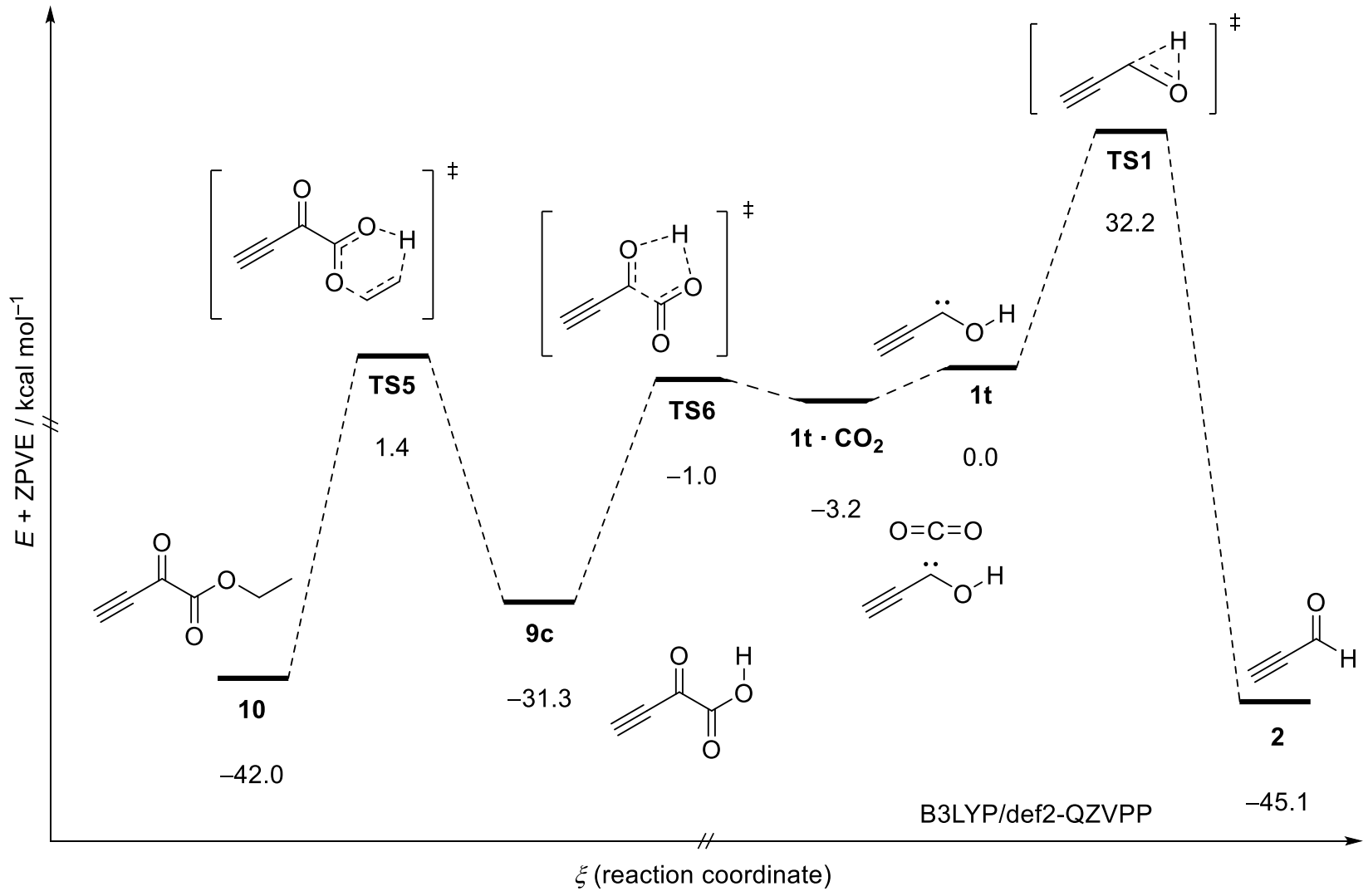

Scheme S2: PES (0 K) of the pyrolysis of ethynylglyoxylic acid ethyl ester (10) computed at the $B 3 L Y P / d e f 2-Q Z V P P$ level of theory. Some energies were corrected for $\mathrm{CO}_{2}$ and ethylene loss. Free $\mathrm{CO}_{2}$ and ethylene were computed at the same level of theory. 


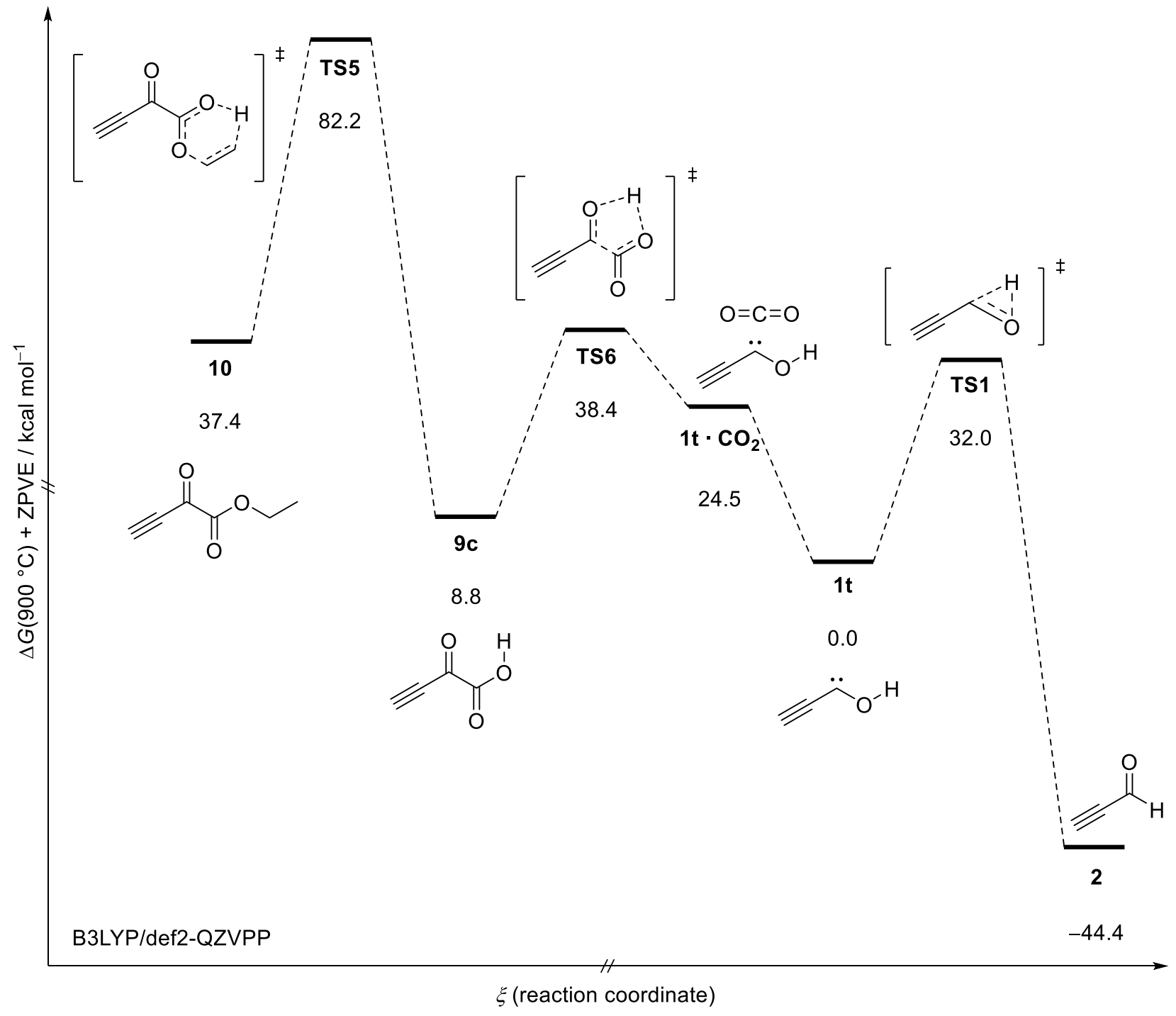

Scheme S3: PES $\left(900{ }^{\circ} \mathrm{C}\right.$ ) of the pyrolysis of ethynylglyoxylic acid ethyl ester (10) computed at the B3LYP/def2-QZVPP level of theory. Some energies were corrected for $\mathrm{CO}_{2}$ and ethylene loss. Free $\mathrm{CO}_{2}$ and ethylene were computed at the same level of theory. 


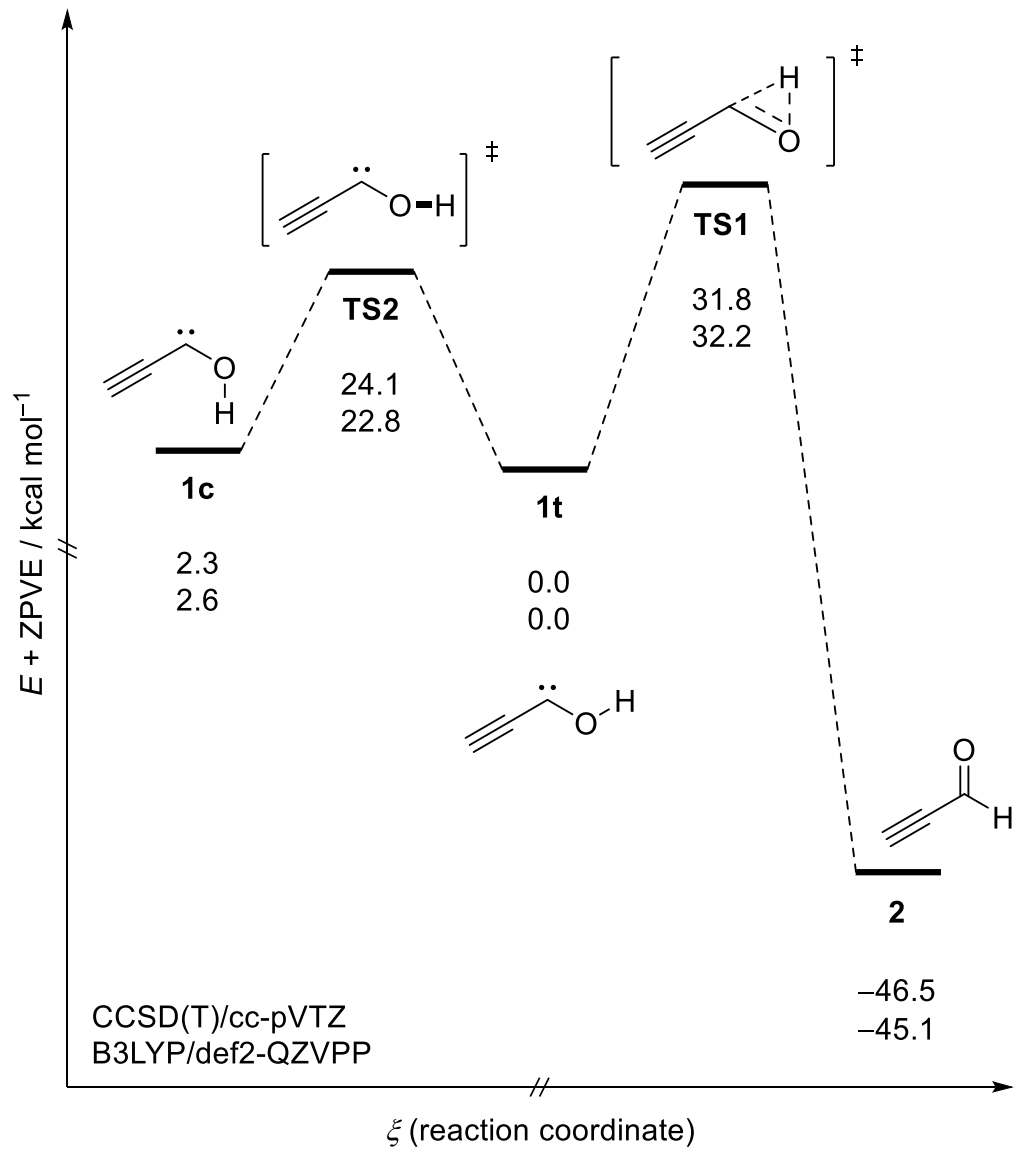

Scheme S4: PES around ethynylhydroxycarbene 1 computed at the CCSD(T)/cc-pVTZ (upper values) and the B3LYP/def2-QZVPP (lower values) levels of theory. 


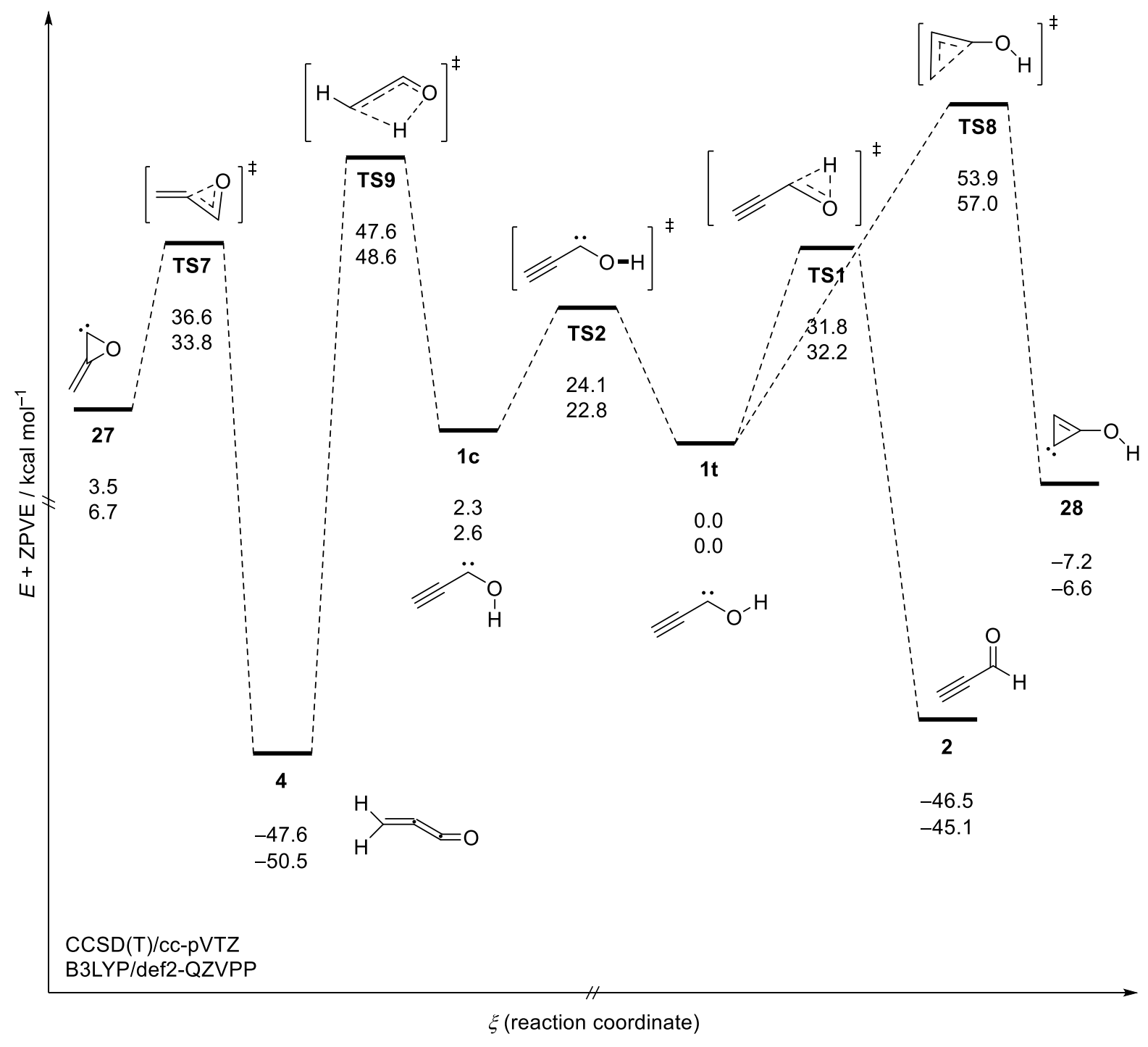

Scheme S5: Expanded PES around ethynylhydroxycarbene 1 computed at the CCSD(T)/cc-pVTZ (upper values) and the B3LYP/def2-QZVPP (lower values) levels of theory. 
<smiles>C#CC(=O)C(=O)OO</smiles>

0.0

11.7

2.1<smiles>C#CC(=O)C(=O)O[Na]</smiles>

3.0<smiles>C#CC(=O)C(=O)O</smiles>

$9 t^{\prime}$

5.8

Scheme S6: Relative energies ( $E+$ ZPVE / kcal mol${ }^{-1}$ ) of the four conformers of ethynylglyoxylic acid (9) computed at the B3LYP/def2-QZVPP level of theory. Only $9 \mathrm{c}$ and $9 \mathrm{t}$ could be observed experimentally. 


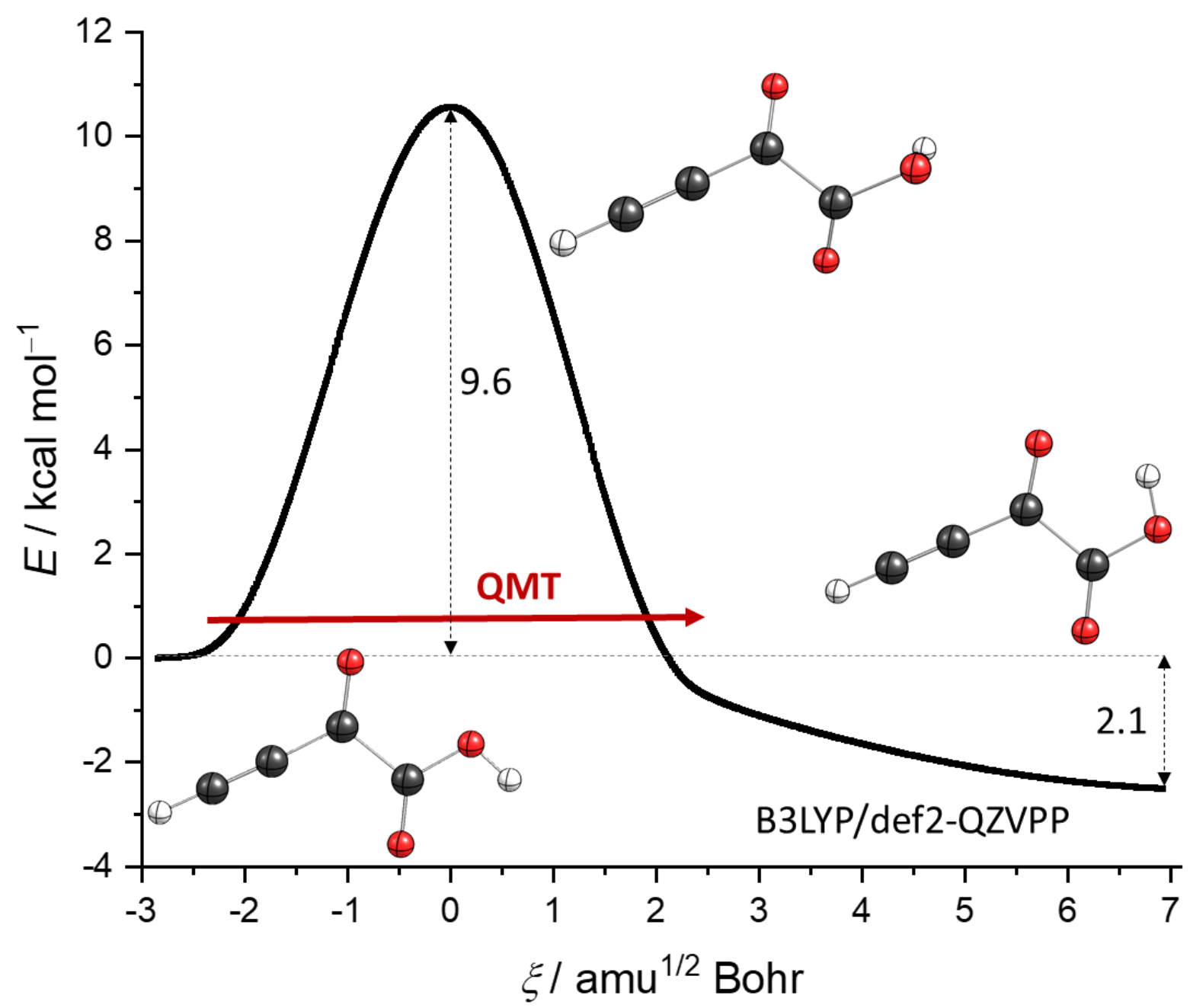

Figure S35: IRC of the reaction 9t $\rightarrow$ 9c computed at the B3LYP/def2-QZVPP level of theory. Note that only the provided relative energies of the stationary points are ZPVE corrected, while the IRC curve is not. 


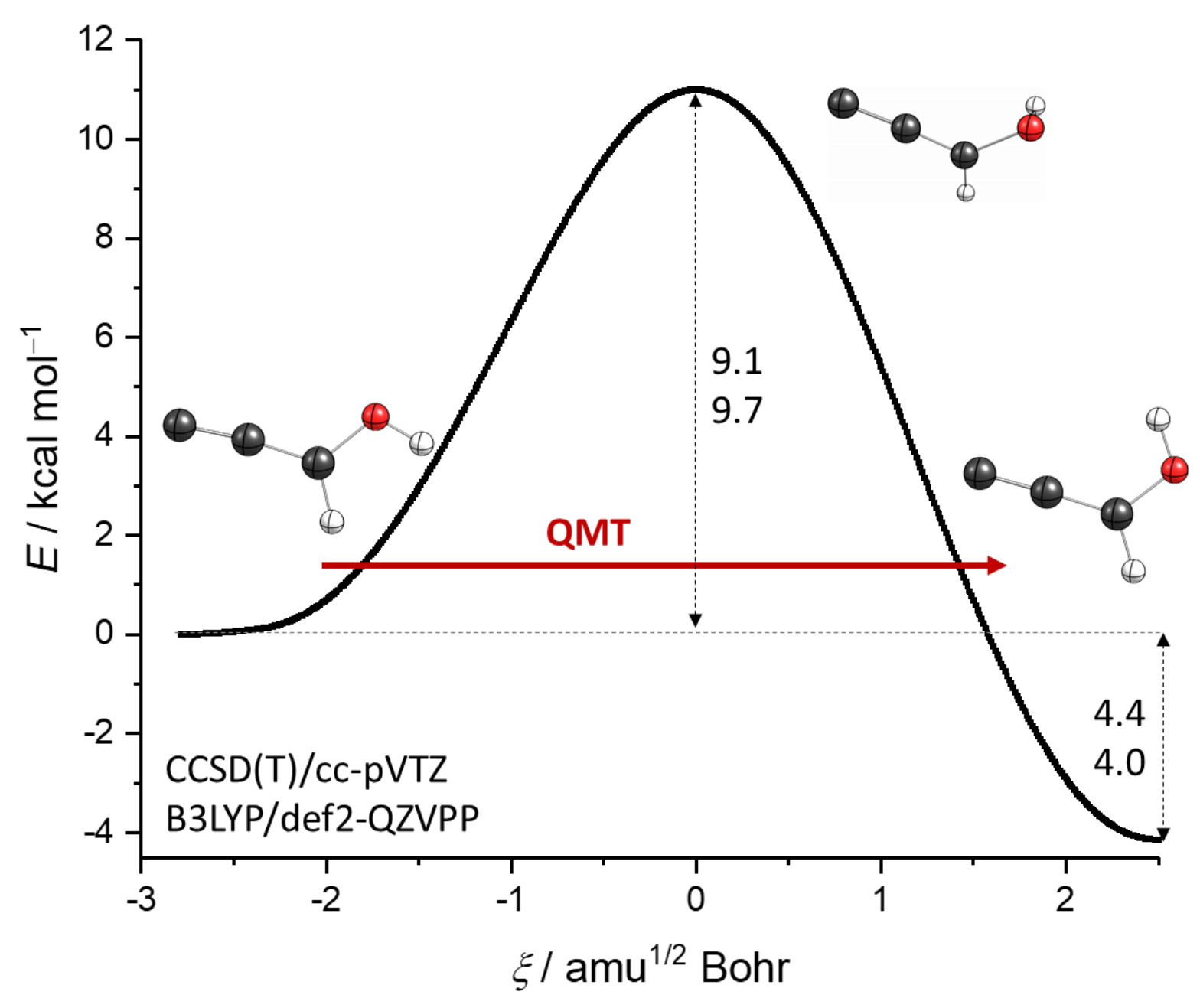

Figure S36: IRC of the reaction $\mathbf{5 c} \rightarrow \mathbf{5 t}$ computed at the B3LYP/def2-QZVPP level of theory. Relative energies are given at the CCSD(T)/cc-pVTZ and the B3LYP/def2-QZVPP levels of theory. Note that only the provided relative energies of the stationary points are ZPVE corrected, while the IRC curve is not. 

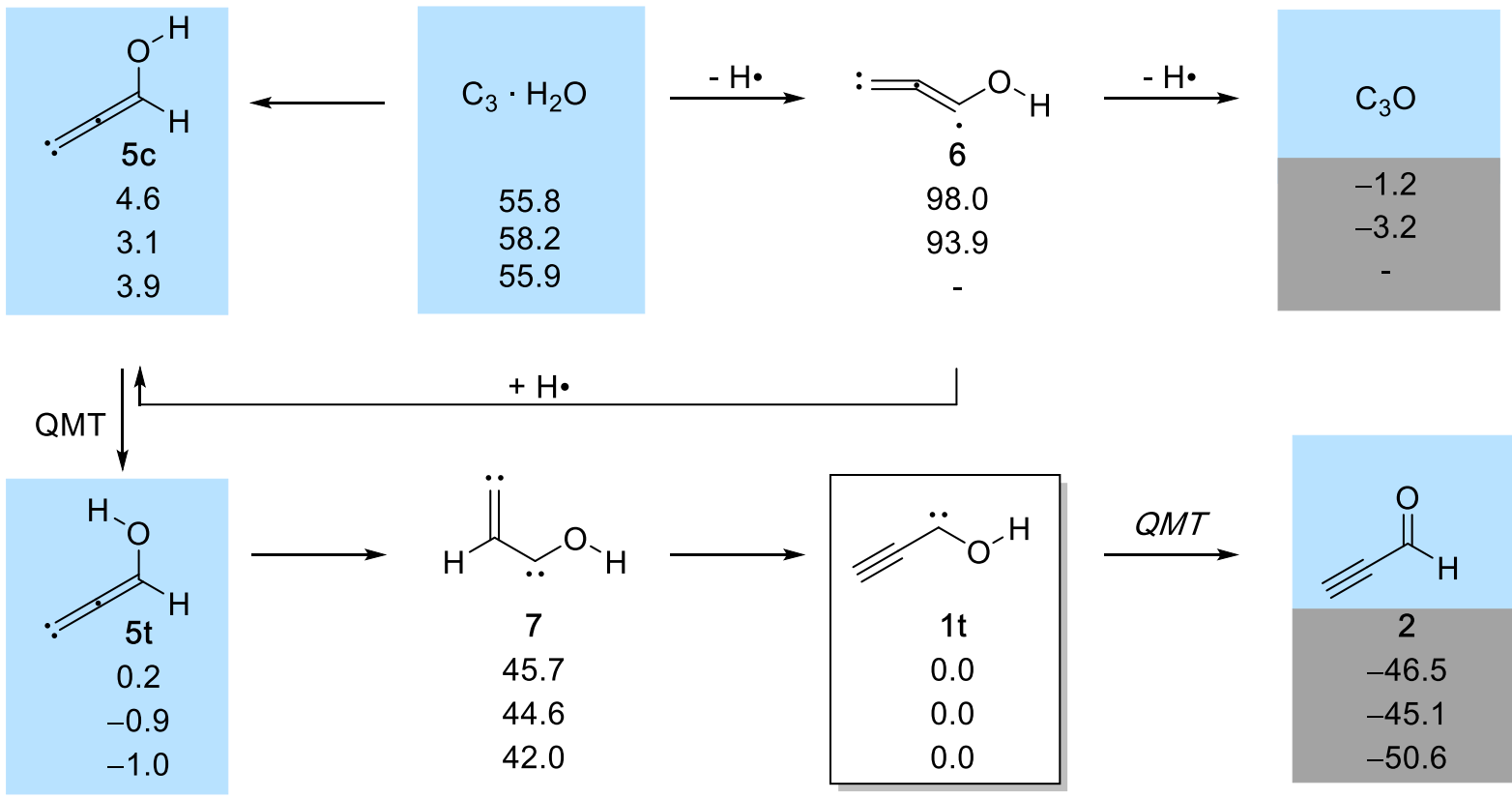

$\operatorname{CCSD}(T) / c c-p V T Z$

B3LYP/def2-QZVPP

QCISD $(T) / 6-31 G$ *

Detected in the laboratory

Detected in space

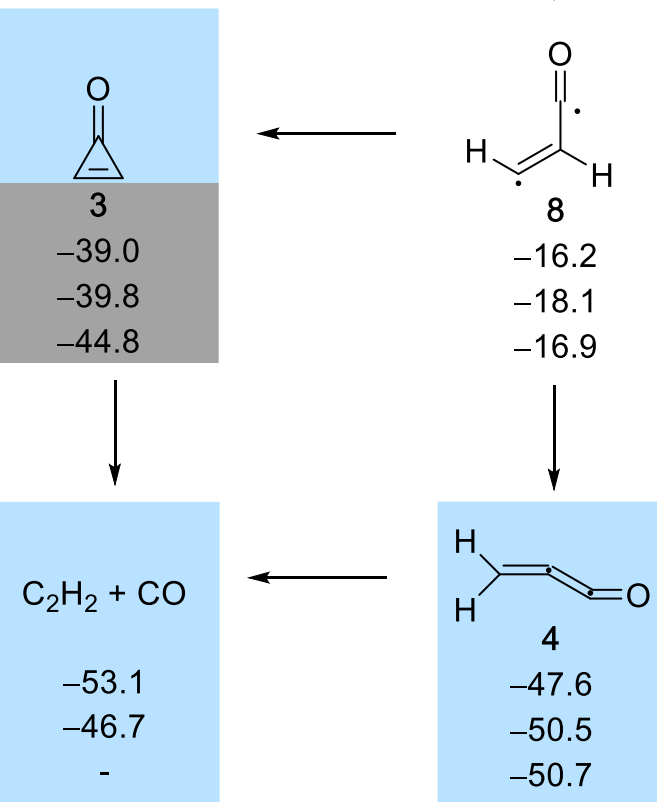

Scheme S7: Proposed mechanism ${ }^{17,18}$ of the observed photoinduced reactions of $\mathrm{C}_{3} \cdot \mathrm{H}_{2} \mathrm{O}$ to $\mathrm{C}_{3} \mathrm{O}, 2$, and acetylene $+\mathrm{CO}^{19}$ Gray: Found in interstellar media. Blue: Characterized under laboratory conditions; $\mathbf{2}$ and $\mathbf{3}$ are commodity chemicals. All compounds that have been found in space have as well been characterized under cryogenic laboratory conditions. Relative energies in kcal mol ${ }^{-1}$ at the QCISD(T)/6-31G* level of theory were obtained from literature data. ${ }^{17}$ The relative energy of 6 is corrected for $\mathrm{H} \cdot$ and the relative energy of $\mathrm{C}_{3} \mathrm{O}$ is corrected for $\mathrm{H}_{2}$. 


\section{Tunneling Computations}

To validate our tunneling computations, we also computed a tunneling half-life for $\mathbf{5 c} \rightarrow \mathbf{5 t}$. This reaction has been reported to proceed very quickly, but no experimental tunneling half-life has been provided by the authors. ${ }^{19}$ Our computed value of $3.11 \mathrm{~s}$ is in agreement with this qualitative result.

Table S21: Overview of computed tunneling half-lives at the CVT/SCT//B3LYP/def2-QZVPP level of theory.

\begin{tabular}{|c|c|c|c|}
\hline Reaction & Symmetry Number & Tunneling Half-Life & Kinetic Isotope Effect \\
\hline $1 t \rightarrow 2$ & 1 & $62.1 \mathrm{~h}$ & \multirow{2}{*}{$9.85 \cdot 10^{6}$} \\
\hline$d_{1}-1 t \rightarrow d_{1}-2$ & 1 & $6.98 \cdot 10^{4} \mathrm{a}$ & \\
\hline $9 t \rightarrow 9 c$ & 2 & $8.60 \mathrm{~h}$ & \multirow{2}{*}{$2.32 \cdot 10^{7}$} \\
\hline$d_{1}-9 t \rightarrow d_{1}-9 c$ & 2 & $2.28 \cdot 10^{4} \mathrm{a}$ & \\
\hline $1 c \rightarrow 1 t$ & 2 & $60.8 d$ & \multirow{2}{*}{$2.92 \cdot 10^{7}$} \\
\hline$d_{1}-1 c \rightarrow d_{1}-1 t$ & 2 & $4.86 \cdot 10^{6} a$ & \\
\hline $5 c \rightarrow 5 t$ & 2 & $3.11 \mathrm{~s}$ & \multirow{2}{*}{$1.99 \cdot 10^{5}$} \\
\hline$d_{1}-5 c \rightarrow d_{1}-5 t$ & 2 & $7.16 \mathrm{~d}$ & \\
\hline
\end{tabular}

Table S22: Rate constants of the reaction $\mathbf{1 t} \rightarrow \mathbf{2}$ in s $\mathrm{s}^{-1}$ computed at the B3LYP/def2-QZVPP level of theory.

\begin{tabular}{lllll}
\hline$T / \mathrm{K}$ & $\mathrm{TST}$ & $\mathrm{CVT}$ & $\mathrm{CVT} / \mathrm{ZCT}$ & $\mathrm{CVT} / \mathrm{SCT}$ \\
\hline 0.10 & $\mathrm{NaN}$ & $\mathrm{NaN}$ & $\mathrm{NaN}$ & $\mathrm{NaN}$ \\
8.00 & $0.00 \cdot 10^{0}$ & $0.00 \cdot 10^{0}$ & $\mathrm{NaN}$ & $\mathrm{NaN}$ \\
10.00 & $0.00 \cdot 10^{0}$ & $0.00 \cdot 10^{0}$ & $\mathrm{NaN}$ & $\mathrm{NaN}$ \\
15.00 & $0.00 \cdot 10^{0}$ & $0.00 \cdot 10^{0}$ & $\mathrm{NaN}$ & $\mathrm{NaN}$ \\
20.00 & $0.00 \cdot 10^{0}$ & $0.00 \cdot 10^{0}$ & $\mathrm{NaN}$ & $\mathrm{NaN}$ \\
25.00 & $2.70 \cdot 10^{-270}$ & $2.69 \cdot 10^{-270}$ & $3.29 \cdot 10^{-7}$ & $3.10 \cdot 10^{-6}$ \\
30.00 & $2.44 \cdot 10^{-223}$ & $2.44 \cdot 10^{-223}$ & $3.29 \cdot 10^{-7}$ & $3.10 \cdot 10^{-6}$ \\
35.00 & $8.70 \cdot 10^{-190}$ & $8.69 \cdot 10^{-190}$ & $3.29 \cdot 10^{-7}$ & $3.10 \cdot 10^{-6}$ \\
40.00 & $1.29 \cdot 10^{-164}$ & $1.29 \cdot 10^{-164}$ & $3.29 \cdot 10^{-7}$ & $3.10 \cdot 10^{-6}$ \\
45.00 & $4.95 \cdot 10^{-145}$ & $4.94 \cdot 10^{-145}$ & $3.30 \cdot 10^{-7}$ & $3.10 \cdot 10^{-6}$ \\
50.00 & $2.33 \cdot 10^{-129}$ & $2.32 \cdot 10^{-129}$ & $3.31 \cdot 10^{-7}$ & $3.11 \cdot 10^{-6}$ \\
200.00 & $2.98 \cdot 10^{-23}$ & $2.98 \cdot 10^{-23}$ & $8.86 \cdot 10^{-7}$ & $6.97 \cdot 10^{-6}$ \\
250.00 & $4.11 \cdot 10^{-16}$ & $4.11 \cdot 10^{-16}$ & $2.37 \cdot 10^{-6}$ & $1.40 \cdot 10^{-5}$ \\
300.00 & $2.45 \cdot 10^{-11}$ & $2.45 \cdot 10^{-11}$ & $1.20 \cdot 10^{-5}$ & $4.64 \cdot 10^{-5}$ \\
350.00 & $6.51 \cdot 10^{-8}$ & $6.50 \cdot 10^{-8}$ & $1.20 \cdot 10^{-4}$ & $3.04 \cdot 10^{-4}$ \\
\hline
\end{tabular}


Table S23: Rate constants of the reaction $\boldsymbol{d}_{\mathbf{1}} \mathbf{- 1 t} \rightarrow \boldsymbol{d}_{\mathbf{1}} \mathbf{- 2}$ in s$^{-1}$ computed at the B3LYP/def2-QZVPP level of theory.

\begin{tabular}{lllll}
\hline$T / \mathrm{K}$ & $\mathrm{TST}$ & $\mathrm{CVT}$ & $\mathrm{CVT} / \mathrm{ZCT}$ & $\mathrm{CVT} / \mathrm{SCT}$ \\
\hline 0.10 & $\mathrm{NaN}$ & $\mathrm{NaN}$ & $\mathrm{NaN}$ & $\mathrm{NaN}$ \\
8.00 & $0.00 \cdot 10^{0}$ & $0.00 \cdot 10^{0}$ & $\mathrm{NaN}$ & $\mathrm{NaN}$ \\
10.00 & $0.00 \cdot 10^{0}$ & $0.00 \cdot 10^{0}$ & $\mathrm{NaN}$ & $\mathrm{NaN}$ \\
15.00 & $0.00 \cdot 10^{0}$ & $0.00 \cdot 10^{0}$ & $\mathrm{NaN}$ & $\mathrm{NaN}$ \\
20.00 & $0.00 \cdot 10^{0}$ & $0.00 \cdot 10^{0}$ & $\mathrm{NaN}$ & $\mathrm{NaN}$ \\
25.00 & $3.03 \cdot 10^{-279}$ & $2.98 \cdot 10^{-279}$ & $8.27 \cdot 10^{-15}$ & $3.18 \cdot 10^{-13}$ \\
30.00 & $8.57 \cdot 10^{-231}$ & $8.45 \cdot 10^{-231}$ & $8.27 \cdot 10^{-15}$ & $3.18 \cdot 10^{-13}$ \\
35.00 & $3.56 \cdot 10^{-196}$ & $3.52 \cdot 10^{-196}$ & $8.27 \cdot 10^{-15}$ & $3.19 \cdot 10^{-13}$ \\
40.00 & $3.33 \cdot 10^{-170}$ & $3.30 \cdot 10^{-170}$ & $8.28 \cdot 10^{-15}$ & $3.19 \cdot 10^{-13}$ \\
45.00 & $5.36 \cdot 10^{-150}$ & $5.31 \cdot 10^{-150}$ & $8.29 \cdot 10^{-15}$ & $3.19 \cdot 10^{-13}$ \\
50.00 & $7.93 \cdot 10^{-134}$ & $7.87 \cdot 10^{-134}$ & $8.32 \cdot 10^{-15}$ & $3.20 \cdot 10^{-13}$ \\
200.00 & $2.38 \cdot 10^{-24}$ & $2.37 \cdot 10^{-24}$ & $8.00 \cdot 10^{-14}$ & $1.52 \cdot 10^{-12}$ \\
250.00 & $5.49 \cdot 10^{-17}$ & $5.48 \cdot 10^{-17}$ & $3.23 \cdot 10^{-12}$ & $2.03 \cdot 10^{-11}$ \\
300.00 & $4.61 \cdot 10^{-12}$ & $4.61 \cdot 10^{-12}$ & $6.64 \cdot 10^{-10}$ & $1.64 \cdot 10^{-9}$ \\
350.00 & $1.55 \cdot 10^{-8}$ & $1.55 \cdot 10^{-8}$ & $2.26 \cdot 10^{-7}$ & $3.22 \cdot 10^{-7}$ \\
\hline
\end{tabular}

Table S24: Rate constants of the reaction $9 \mathrm{t} \rightarrow \mathbf{9 c}$ in s $\mathrm{s}^{-1}$ computed at the B3LYP/def2-QZPVP level of theory.

\begin{tabular}{lllll}
\hline$T / \mathrm{K}$ & $\mathrm{TST}$ & $\mathrm{CVT}$ & $\mathrm{CVT} / \mathrm{ZCT}$ & $\mathrm{CVT} / \mathrm{SCT}$ \\
\hline 0.10 & $\mathrm{NaN}$ & $\mathrm{NaN}$ & $\mathrm{NaN}$ & $\mathrm{NaN}$ \\
8.00 & $4.55 \cdot 10^{-253}$ & $4.55 \cdot 10^{-253}$ & $7.56 \cdot 10^{-8}$ & $2.24 \cdot 10^{-5}$ \\
10.00 & $3.36 \cdot 10^{-200}$ & $3.36 \cdot 10^{-200}$ & $7.63 \cdot 10^{-8}$ & $2.26 \cdot 10^{-5}$ \\
15.00 & $1.13 \cdot 10^{-129}$ & $1.13 \cdot 10^{-129}$ & $8.02 \cdot 10^{-8}$ & $2.36 \cdot 10^{-5}$ \\
20.00 & $2.24 \cdot 10^{-94}$ & $2.24 \cdot 10^{-94}$ & $8.73 \cdot 10^{-8}$ & $2.56 \cdot 10^{-5}$ \\
25.00 & $3.50 \cdot 10^{-73}$ & $3.50 \cdot 10^{-73}$ & $9.77 \cdot 10^{-8}$ & $2.84 \cdot 10^{-5}$ \\
30.00 & $4.86 \cdot 10^{-59}$ & $4.86 \cdot 10^{-59}$ & $1.12 \cdot 10^{-7}$ & $3.21 \cdot 10^{-5}$ \\
35.00 & $6.28 \cdot 10^{-49}$ & $6.28 \cdot 10^{-49}$ & $1.30 \cdot 10^{-7}$ & $3.67 \cdot 10^{-5}$ \\
40.00 & $2.45 \cdot 10^{-41}$ & $2.45 \cdot 10^{-41}$ & $1.53 \cdot 10^{-7}$ & $4.23 \cdot 10^{-5}$ \\
45.00 & $1.99 \cdot 10^{-35}$ & $1.99 \cdot 10^{-35}$ & $1.83 \cdot 10^{-7}$ & $4.90 \cdot 10^{-5}$ \\
50.00 & $1.07 \cdot 10^{-30}$ & $1.07 \cdot 10^{-30}$ & $2.24 \cdot 10^{-7}$ & $5.70 \cdot 10^{-5}$ \\
200.00 & $1.87 \cdot 10^{2}$ & $1.87 \cdot 10^{2}$ & $7.39 \cdot 10^{2}$ & $1.37 \cdot 10^{3}$ \\
250.00 & $2.98 \cdot 10^{4}$ & $2.98 \cdot 10^{4}$ & $7.03 \cdot 10^{4}$ & $1.04 \cdot 10^{5}$ \\
300.00 & $8.97 \cdot 10^{5}$ & $8.97 \cdot 10^{5}$ & $1.62 \cdot 10^{6}$ & $2.12 \cdot 10^{6}$ \\
350.00 & $1.04 \cdot 10^{7}$ & $1.04 \cdot 10^{7}$ & $1.59 \cdot 10^{7}$ & $1.94 \cdot 10^{7}$ \\
\hline
\end{tabular}


Table S25: Rate constants of the reaction $\boldsymbol{d}_{1}-9 t \rightarrow \boldsymbol{d}_{1}-9 \mathrm{c}$ in s ${ }^{-1}$ computed at the B3LYP/def2-QZVPP level of theory.

\begin{tabular}{lllll}
\hline$T / \mathrm{K}$ & $\mathrm{TST}$ & $\mathrm{CVT}$ & $\mathrm{CVT} / \mathrm{ZCT}$ & $\mathrm{CVT} / \mathrm{SCT}$ \\
\hline 0.10 & $\mathrm{NaN}$ & $\mathrm{NaN}$ & $\mathrm{NaN}$ & $\mathrm{NaN}$ \\
8.00 & $5.25 \cdot 10^{-260}$ & $6.71 \cdot 10^{-260}$ & $7.17 \cdot 10^{-17}$ & $9.64 \cdot 10^{-13}$ \\
10.00 & $9.45 \cdot 10^{-206}$ & $1.15 \cdot 10^{-205}$ & $8.49 \cdot 10^{-17}$ & $1.14 \cdot 10^{-12}$ \\
15.00 & $2.26 \cdot 10^{-133}$ & $2.58 \cdot 10^{-133}$ & $1.11 \cdot 10^{-16}$ & $1.49 \cdot 10^{-12}$ \\
20.00 & $3.75 \cdot 10^{-97}$ & $4.14 \cdot 10^{-97}$ & $1.38 \cdot 10^{-16}$ & $1.81 \cdot 10^{-12}$ \\
25.00 & $2.11 \cdot 10^{-75}$ & $2.28 \cdot 10^{-75}$ & $1.69 \cdot 10^{-16}$ & $2.18 \cdot 10^{-12}$ \\
30.00 & $6.88 \cdot 10^{-61}$ & $7.34 \cdot 10^{-61}$ & $2.08 \cdot 10^{-16}$ & $2.62 \cdot 10^{-12}$ \\
35.00 & $1.63 \cdot 10^{-50}$ & $1.73 \cdot 10^{-50}$ & $2.63 \cdot 10^{-16}$ & $3.16 \cdot 10^{-12}$ \\
40.00 & $1.01 \cdot 10^{-42}$ & $1.06 \cdot 10^{-42}$ & $3.49 \cdot 10^{-16}$ & $3.84 \cdot 10^{-12}$ \\
45.00 & $1.16 \cdot 10^{-36}$ & $1.22 \cdot 10^{-36}$ & $5.06 \cdot 10^{-16}$ & $4.74 \cdot 10^{-12}$ \\
50.00 & $8.36 \cdot 10^{-32}$ & $8.69 \cdot 10^{-32}$ & $8.37 \cdot 10^{-16}$ & $6.04 \cdot 10^{-12}$ \\
200.00 & $9.62 \cdot 10^{1}$ & $9.72 \cdot 10^{1}$ & $2.19 \cdot 10^{2}$ & $3.20 \cdot 10^{2}$ \\
250.00 & $1.71 \cdot 10^{4}$ & $1.72 \cdot 10^{4}$ & $2.87 \cdot 10^{4}$ & $3.67 \cdot 10^{4}$ \\
300.00 & $5.50 \cdot 10^{5}$ & $5.53 \cdot 10^{5}$ & $7.82 \cdot 10^{5}$ & $9.33 \cdot 10^{5}$ \\
350.00 & $6.63 \cdot 10^{6}$ & $6.67 \cdot 10^{6}$ & $8.55 \cdot 10^{6}$ & $9.75 \cdot 10^{6}$ \\
\hline
\end{tabular}

Table S26: Rate constants of the reaction $\mathbf{1 c} \rightarrow \mathbf{1 t}$ in $\mathrm{s}^{-1}$ computed at the B3LYP/def2-QZVPP level of theory.

\begin{tabular}{lllll}
\hline$T / \mathrm{K}$ & $\mathrm{TST}$ & $\mathrm{CVT}$ & $\mathrm{CVT} / \mathrm{ZCT}$ & $\mathrm{CVT} / \mathrm{SCT}$ \\
\hline 0.10 & $\mathrm{NaN}$ & $\mathrm{NaN}$ & $\mathrm{NaN}$ & $\mathrm{NaN}$ \\
8.00 & $0.00 \cdot 10^{0}$ & $0.00 \cdot 10^{0}$ & $\mathrm{NaN}$ & $\mathrm{NaN}$ \\
10.00 & $0.00 \cdot 10^{0}$ & $0.00 \cdot 10^{0}$ & $\mathrm{NaN}$ & $\mathrm{NaN}$ \\
15.00 & $2.31 \cdot 10^{-283}$ & $2.30 \cdot 10^{-283}$ & $5.49 \cdot 10^{-11}$ & $1.32 \cdot 10^{-7}$ \\
20.00 & $1.25 \cdot 10^{-209}$ & $1.25 \cdot 10^{-209}$ & $5.49 \cdot 10^{-11}$ & $1.32 \cdot 10^{-7}$ \\
25.00 & $2.30 \cdot 10^{-165}$ & $2.29 \cdot 10^{-165}$ & $5.50 \cdot 10^{-11}$ & $1.32 \cdot 10^{-7}$ \\
30.00 & $7.66 \cdot 10^{-136}$ & $7.64 \cdot 10^{-136}$ & $5.50 \cdot 10^{-11}$ & $1.32 \cdot 10^{-7}$ \\
35.00 & $9.61 \cdot 10^{-115}$ & $9.58 \cdot 10^{-115}$ & $5.51 \cdot 10^{-11}$ & $1.32 \cdot 10^{-7}$ \\
40.00 & $6.52 \cdot 10^{-99}$ & $6.50 \cdot 10^{-99}$ & $5.52 \cdot 10^{-11}$ & $1.32 \cdot 10^{-7}$ \\
45.00 & $1.36 \cdot 10^{-86}$ & $1.36 \cdot 10^{-86}$ & $5.54 \cdot 10^{-11}$ & $1.33 \cdot 10^{-7}$ \\
50.00 & $9.87 \cdot 10^{-77}$ & $9.85 \cdot 10^{-77}$ & $5.58 \cdot 10^{-11}$ & $1.33 \cdot 10^{-7}$ \\
200.00 & $7.33 \cdot 10^{-10}$ & $7.33 \cdot 10^{-10}$ & $2.56 \cdot 10^{-7}$ & $1.04 \cdot 10^{-5}$ \\
250.00 & $2.43 \cdot 10^{-5}$ & $2.43 \cdot 10^{-5}$ & $3.05 \cdot 10^{-4}$ & $1.85 \cdot 10^{-3}$ \\
300.00 & $2.60 \cdot 10^{-2}$ & $2.60 \cdot 10^{-2}$ & $1.10 \cdot 10^{-1}$ & $3.14 \cdot 10^{-1}$ \\
350.00 & $3.88 \cdot 10^{0}$ & $3.88 \cdot 10^{0}$ & $9.96 \cdot 10^{0}$ & $2.01 \cdot 10^{1}$ \\
\hline
\end{tabular}


Table S27: Rate constants of the reaction $\boldsymbol{d}_{\mathbf{1}} \mathbf{- 1 c} \rightarrow \boldsymbol{d}_{\mathbf{1}}-\mathbf{1 t}$ in $\mathrm{s}^{-1}$ computed at the B3LYP/def2-QZVPP level of theory.

\begin{tabular}{lllll}
\hline$T / \mathrm{K}$ & $\mathrm{TST}$ & $\mathrm{CVT}$ & $\mathrm{CVT} / \mathrm{ZCT}$ & $\mathrm{CVT} / \mathrm{SCT}$ \\
\hline 0.10 & $\mathrm{NaN}$ & $\mathrm{NaN}$ & $\mathrm{NaN}$ & $\mathrm{NaN}$ \\
8.00 & $0.00 \cdot 10^{0}$ & $0.00 \cdot 10^{0}$ & $\mathrm{NaN}$ & $\mathrm{NaN}$ \\
10.00 & $0.00 \cdot 10^{0}$ & $0.00 \cdot 10^{0}$ & $\mathrm{NaN}$ & $\mathrm{NaN}$ \\
15.00 & $2.54 \cdot 10^{-287}$ & $2.53 \cdot 10^{-287}$ & $1.96 \cdot 10^{-18}$ & $4.52 \cdot 10^{-15}$ \\
20.00 & $1.34 \cdot 10^{-212}$ & $1.34 \cdot 10^{-212}$ & $1.96 \cdot 10^{-18}$ & $4.52 \cdot 10^{-15}$ \\
25.00 & $9.64 \cdot 10^{-168}$ & $9.63 \cdot 10^{-168}$ & $1.96 \cdot 10^{-18}$ & $4.52 \cdot 10^{-15}$ \\
30.00 & $7.99 \cdot 10^{-138}$ & $7.98 \cdot 10^{-138}$ & $1.96 \cdot 10^{-18}$ & $4.52 \cdot 10^{-15}$ \\
35.00 & $1.92 \cdot 10^{-116}$ & $1.92 \cdot 10^{-116}$ & $1.97 \cdot 10^{-18}$ & $4.53 \cdot 10^{-15}$ \\
40.00 & $2.12 \cdot 10^{-100}$ & $2.12 \cdot 10^{-100}$ & $1.97 \cdot 10^{-18}$ & $4.54 \cdot 10^{-15}$ \\
45.00 & $6.48 \cdot 10^{-88}$ & $6.47 \cdot 10^{-88}$ & $1.99 \cdot 10^{-18}$ & $4.57 \cdot 10^{-15}$ \\
50.00 & $6.36 \cdot 10^{-78}$ & $6.36 \cdot 10^{-78}$ & $2.01 \cdot 10^{-18}$ & $4.61 \cdot 10^{-15}$ \\
200.00 & $3.64 \cdot 10^{-10}$ & $3.64 \cdot 10^{-10}$ & $4.33 \cdot 10^{-9}$ & $2.52 \cdot 10^{-8}$ \\
250.00 & $1.38 \cdot 10^{-5}$ & $1.38 \cdot 10^{-5}$ & $5.13 \cdot 10^{-5}$ & $1.22 \cdot 10^{-4}$ \\
300.00 & $1.61 \cdot 10^{-2}$ & $1.61 \cdot 10^{-2}$ & $3.78 \cdot 10^{-2}$ & $6.55 \cdot 10^{-2}$ \\
350.00 & $2.55 \cdot 10^{0}$ & $2.55 \cdot 10^{0}$ & $4.71 \cdot 10^{0}$ & $6.93 \cdot 10^{0}$ \\
\hline
\end{tabular}

Table S28: Rate constants of the reaction $\mathbf{5 c} \rightarrow \mathbf{5 t}$ in s$^{-1}$ computed at the B3LYP/def2-QZVPP level of theory.

\begin{tabular}{lllll}
\hline$T / \mathrm{K}$ & TST & CVT & CVT/ZCT & CVT/SCT \\
\hline 0.10 & NaN & NaN & NaN & NaN \\
8.00 & $4.96 \cdot 10^{-254}$ & $4.96 \cdot 10^{-254}$ & $6.78 \cdot 10^{-4}$ & $2.23 \cdot 10^{-1}$ \\
10.00 & $5.81 \cdot 10^{-201}$ & $5.81 \cdot 10^{-201}$ & $6.78 \cdot 10^{-4}$ & $2.24 \cdot 10^{-1}$ \\
15.00 & $3.71 \cdot 10^{-130}$ & $3.71 \cdot 10^{-130}$ & $6.79 \cdot 10^{-4}$ & $2.24 \cdot 10^{-1}$ \\
20.00 & $1.02 \cdot 10^{-94}$ & $1.02 \cdot 10^{-94}$ & $6.79 \cdot 10^{-4}$ & $2.24 \cdot 10^{-1}$ \\
25.00 & $1.97 \cdot 10^{-73}$ & $1.97 \cdot 10^{-73}$ & $6.80 \cdot 10^{-4}$ & $2.24 \cdot 10^{-1}$ \\
30.00 & $3.15 \cdot 10^{-59}$ & $3.16 \cdot 10^{-59}$ & $6.81 \cdot 10^{-4}$ & $2.24 \cdot 10^{-1}$ \\
35.00 & $4.53 \cdot 10^{-49}$ & $4.53 \cdot 10^{-49}$ & $6.84 \cdot 10^{-4}$ & $2.25 \cdot 10^{-1}$ \\
40.00 & $1.91 \cdot 10^{-41}$ & $1.91 \cdot 10^{-41}$ & $6.90 \cdot 10^{-4}$ & $2.26 \cdot 10^{-1}$ \\
45.00 & $1.65 \cdot 10^{-35}$ & $1.65 \cdot 10^{-35}$ & $7.01 \cdot 10^{-4}$ & $2.27 \cdot 10^{-1}$ \\
50.00 & $9.37 \cdot 10^{-31}$ & $9.38 \cdot 10^{-31}$ & $7.20 \cdot 10^{-4}$ & $2.30 \cdot 10^{-1}$ \\
200.00 & $2.20 \cdot 10^{2}$ & $2.20 \cdot 10^{2}$ & $1.23 \cdot 10^{3}$ & $2.57 \cdot 10^{3}$ \\
250.00 & $3.54 \cdot 10^{4}$ & $3.54 \cdot 10^{4}$ & $1.02 \cdot 10^{5}$ & $1.57 \cdot 10^{5}$ \\
300.00 & $1.07 \cdot 10^{6}$ & $1.07 \cdot 10^{6}$ & $2.20 \cdot 10^{6}$ & $2.95 \cdot 10^{6}$ \\
350.00 & $1.24 \cdot 10^{7}$ & $1.24 \cdot 10^{7}$ & $2.09 \cdot 10^{7}$ & $2.60 \cdot 10^{7}$ \\
\hline
\end{tabular}


Table S29: Rate constants of the reaction $\boldsymbol{d}_{1}-5 \mathrm{c} \rightarrow \boldsymbol{d}_{1}-5 \mathrm{t}$ in $\mathrm{s}^{-1}$ computed at the B3LYP/def2-QZVPP level of theory.

\begin{tabular}{lllll}
\hline$T / \mathrm{K}$ & $\mathrm{TST}$ & $\mathrm{CVT}$ & $\mathrm{CVT} / \mathrm{ZCT}$ & $\mathrm{CVT} / \mathrm{SCT}$ \\
\hline 0.10 & $\mathrm{NaN}$ & $\mathrm{NaN}$ & $\mathrm{NaN}$ & $\mathrm{NaN}$ \\
8.00 & $1.24 \cdot 10^{-261}$ & $1.24 \cdot 10^{-261}$ & $1.21 \cdot 10^{-9}$ & $1.12 \cdot 10^{-6}$ \\
10.00 & $4.86 \cdot 10^{-207}$ & $4.86 \cdot 10^{-207}$ & $1.21 \cdot 10^{-9}$ & $1.12 \cdot 10^{-6}$ \\
15.00 & $3.33 \cdot 10^{-134}$ & $3.33 \cdot 10^{-134}$ & $1.21 \cdot 10^{-9}$ & $1.12 \cdot 10^{-6}$ \\
20.00 & $9.50 \cdot 10^{-98}$ & $9.50 \cdot 10^{-98}$ & $1.21 \cdot 10^{-9}$ & $1.12 \cdot 10^{-6}$ \\
25.00 & $7.46 \cdot 10^{-76}$ & $7.46 \cdot 10^{-76}$ & $1.21 \cdot 10^{-9}$ & $1.12 \cdot 10^{-6}$ \\
30.00 & $3.04 \cdot 10^{-61}$ & $3.04 \cdot 10^{-61}$ & $1.21 \cdot 10^{-9}$ & $1.12 \cdot 10^{-6}$ \\
35.00 & $8.52 \cdot 10^{-51}$ & $8.52 \cdot 10^{-51}$ & $1.23 \cdot 10^{-9}$ & $1.13 \cdot 10^{-6}$ \\
40.00 & $5.93 \cdot 10^{-43}$ & $5.93 \cdot 10^{-43}$ & $1.25 \cdot 10^{-9}$ & $1.14 \cdot 10^{-6}$ \\
45.00 & $7.57 \cdot 10^{-37}$ & $7.57 \cdot 10^{-37}$ & $1.30 \cdot 10^{-9}$ & $1.15 \cdot 10^{-6}$ \\
50.00 & $5.87 \cdot 10^{-32}$ & $5.87 \cdot 10^{-32}$ & $1.39 \cdot 10^{-9}$ & $1.19 \cdot 10^{-6}$ \\
200.00 & $1.09 \cdot 10^{2}$ & $1.09 \cdot 10^{2}$ & $3.04 \cdot 10^{2}$ & $4.66 \cdot 10^{2}$ \\
250.00 & $1.98 \cdot 10^{4}$ & $1.98 \cdot 10^{4}$ & $3.87 \cdot 10^{4}$ & $5.04 \cdot 10^{4}$ \\
300.00 & $6.46 \cdot 10^{5}$ & $6.46 \cdot 10^{5}$ & $1.04 \cdot 10^{6}$ & $1.25 \cdot 10^{6}$ \\
350.00 & $7.88 \cdot 10^{6}$ & $7.88 \cdot 10^{6}$ & $1.13 \cdot 10^{7}$ & $1.30 \cdot 10^{7}$ \\
\hline
\end{tabular}




\section{NBO Analysis}

In the following we present our results of the NBO and NRT analyses as well as Wiberg bond indices for $\mathbf{1 t}$ and $\mathbf{1 c}$. For both compounds only $\pi$-type orbitals and their antibonding analogues are shown along with the carbene electron lone pair (HOMO). Lower lying occupied orbitals represent either $\sigma$ bonding or s-type orbitals.

trans-Ethynylhydroxycarbene $\mathbf{1 t}$

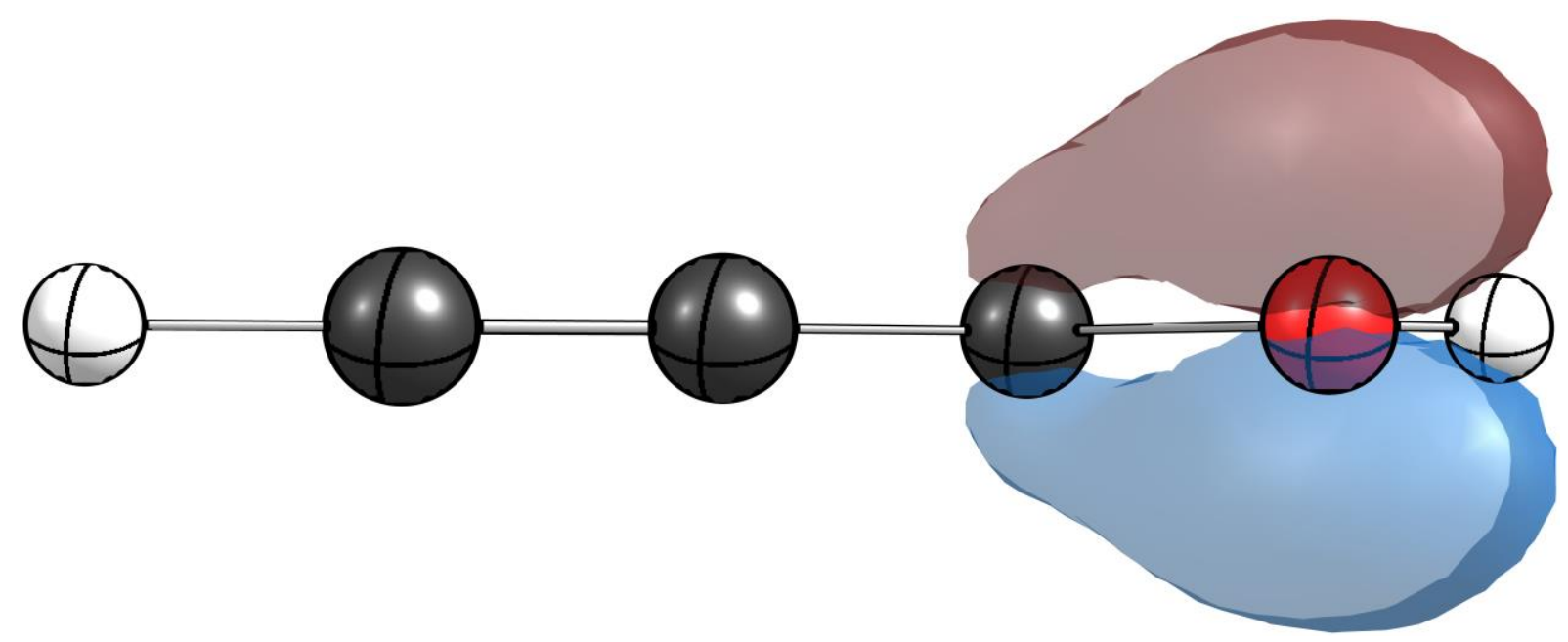

Figure S371: Depiction of the HOMO-3 of 1t. The NBO resembles a $\pi$ orbital along the C-O bond. It can be considered as the bonding combination of the oxygen electron lone pair with the empty p-orbital at the carbene center.

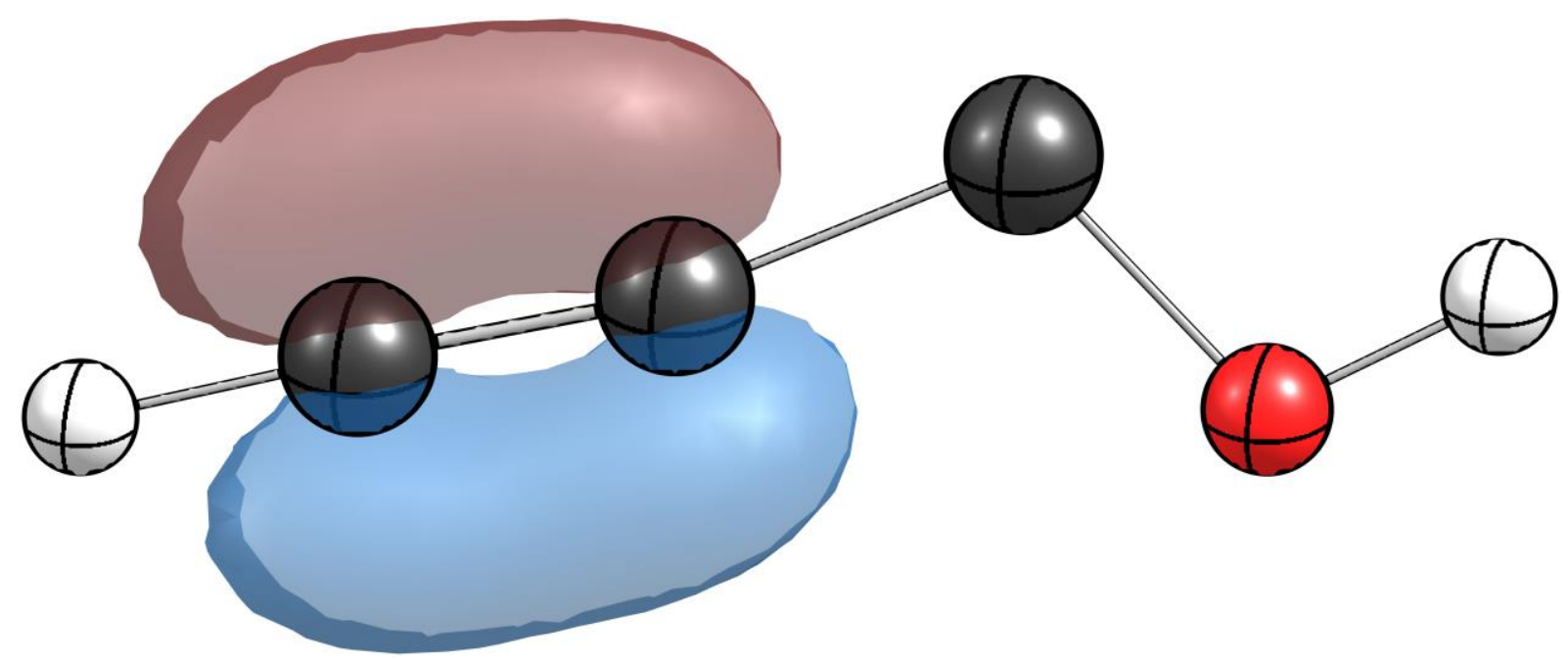

Figure S382: Depiction of the HOMO-2 of 1t. The NBO resembles the in-plane $\pi$-bond at the alkyne moiety. 


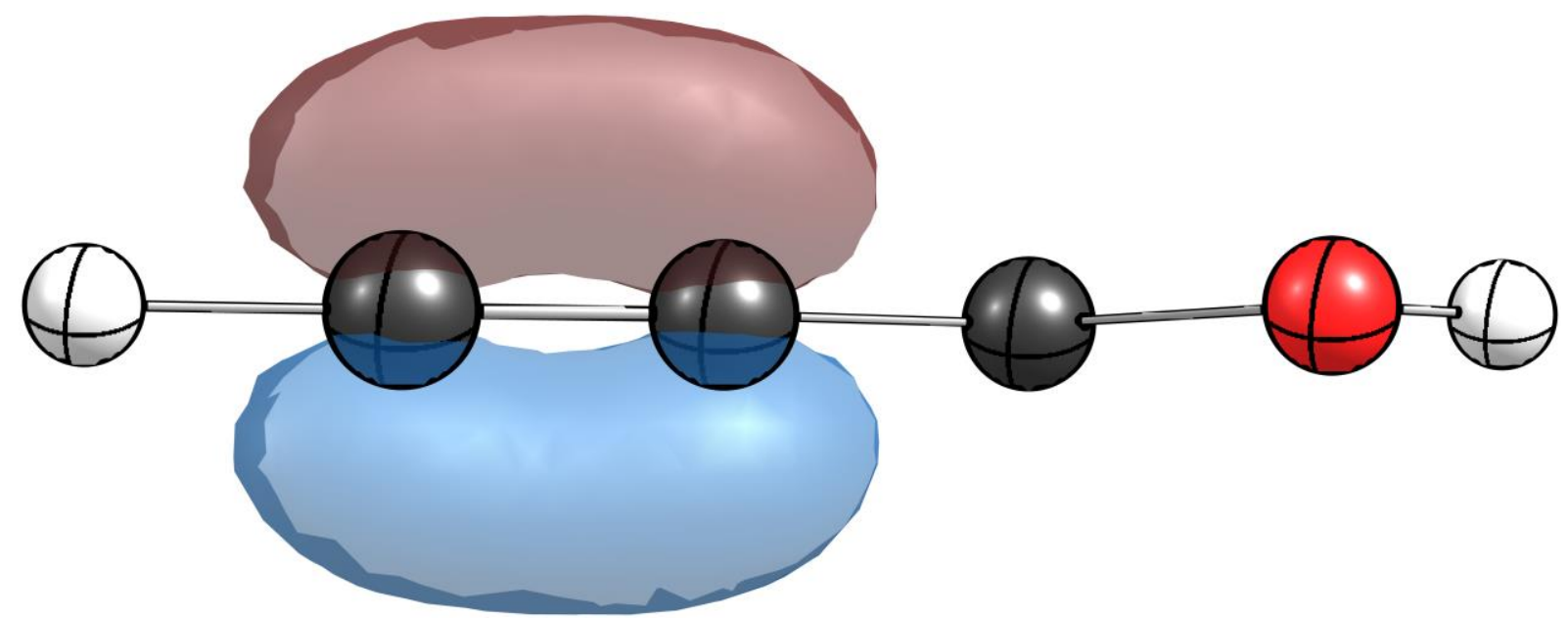

Figure S39: Depiction of the HOMO-1 of $1 \mathbf{t}$. The NBO resembles the out-of-plane $\pi$-bond at the alkyne moiety.

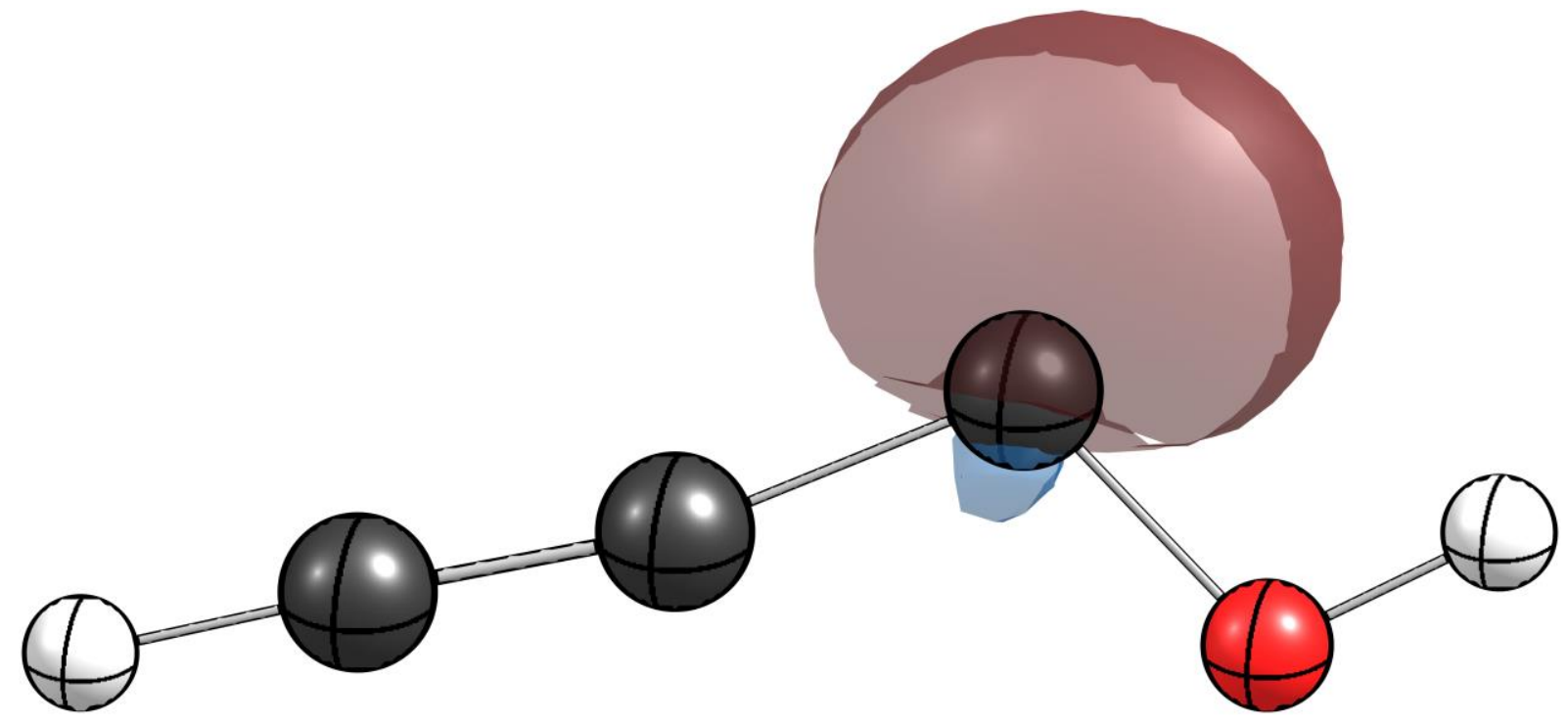

Figure S403: Depiction of the HOMO of 1t. The NBO resembles the electron lone pair at the carbene center.

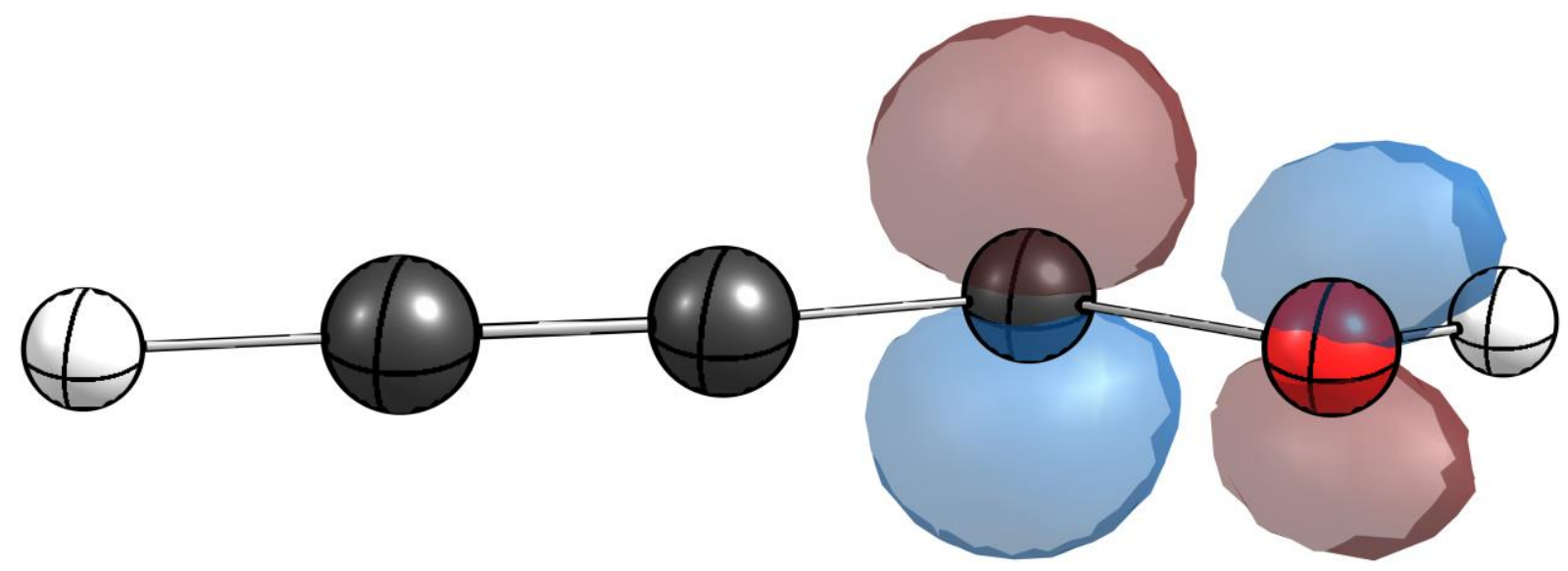

Figure S41: Depiction of the LUMO of $1 \mathrm{t}$. The NBO resembles a $\pi^{*}$ orbital along the C-O bond. It can be considered as the antibonding combination of the oxygen electron lone pair with the empty p-orbital at the carbene center. 


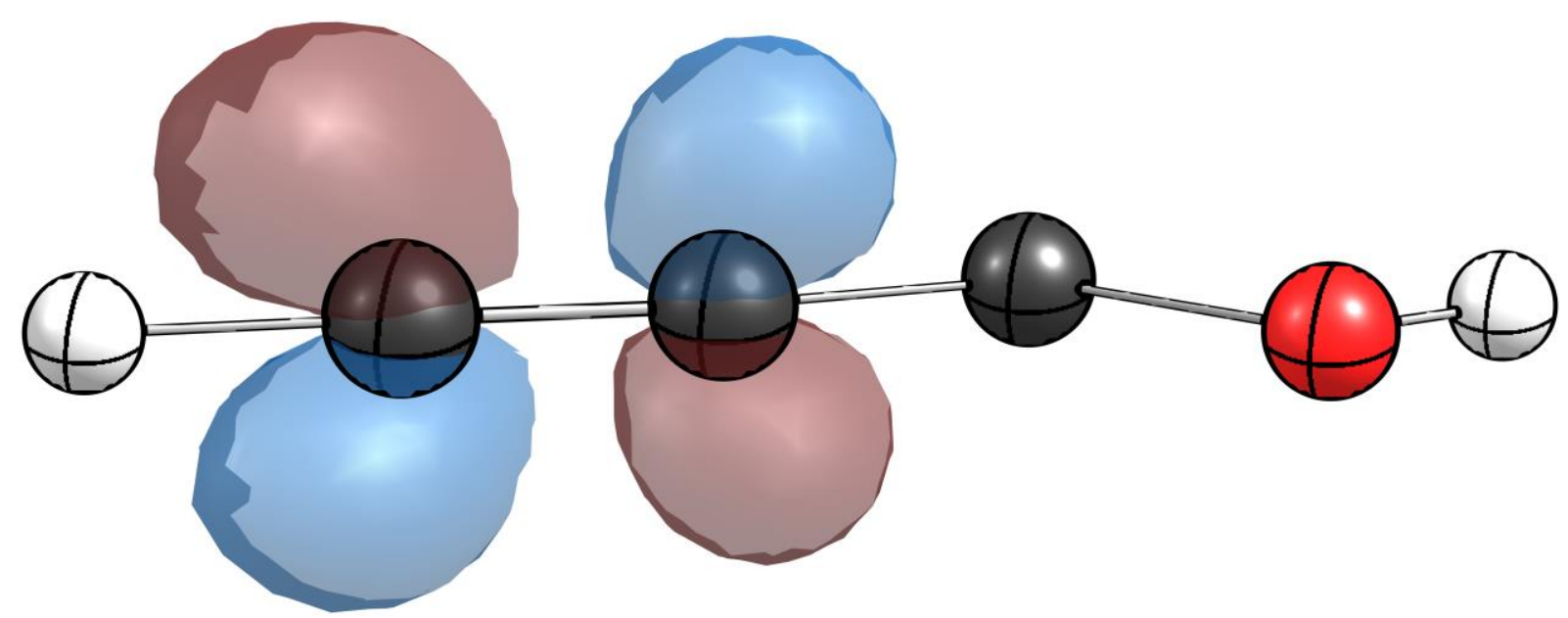

Figure S42: Depiction of the LUMO+1 of 1t. The NBO resembles the out-of-plane $\pi^{*}$ bond at the alkyne moiety.

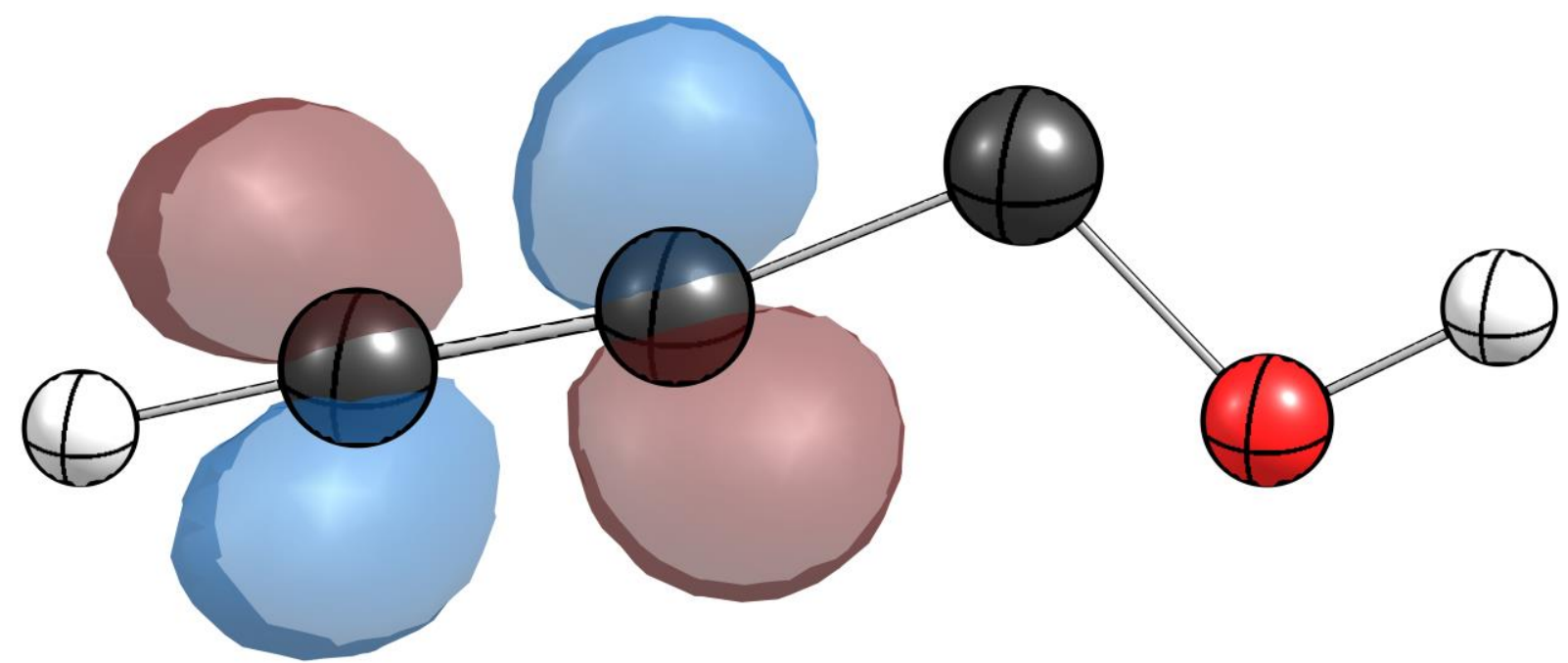

Figure S43: Depiction of the LUMO+2 of $1 \mathrm{t}$. The NBO resembles the in-plane alkyne $\pi^{*}$ bond at the alkyne moiety.

NRT Analysis:

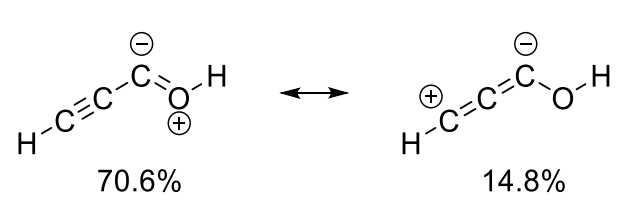

Wiberg Bond Indices:

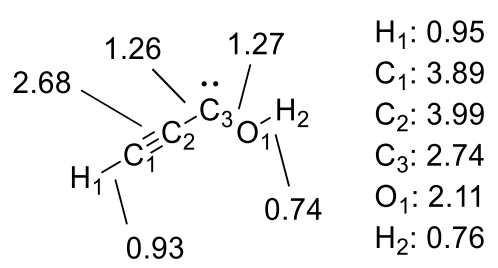

Figure S44: Natural resonance theory (NRT) analysis of trans-ethynylhydroxycarbene 1 t at B3LYP/def2QZVPP based on the optimized geometry of $\mathbf{1 t}$ at the same level of theory. Only the most relevant mesomeric structures contributing more than $5 \%$ are shown. 


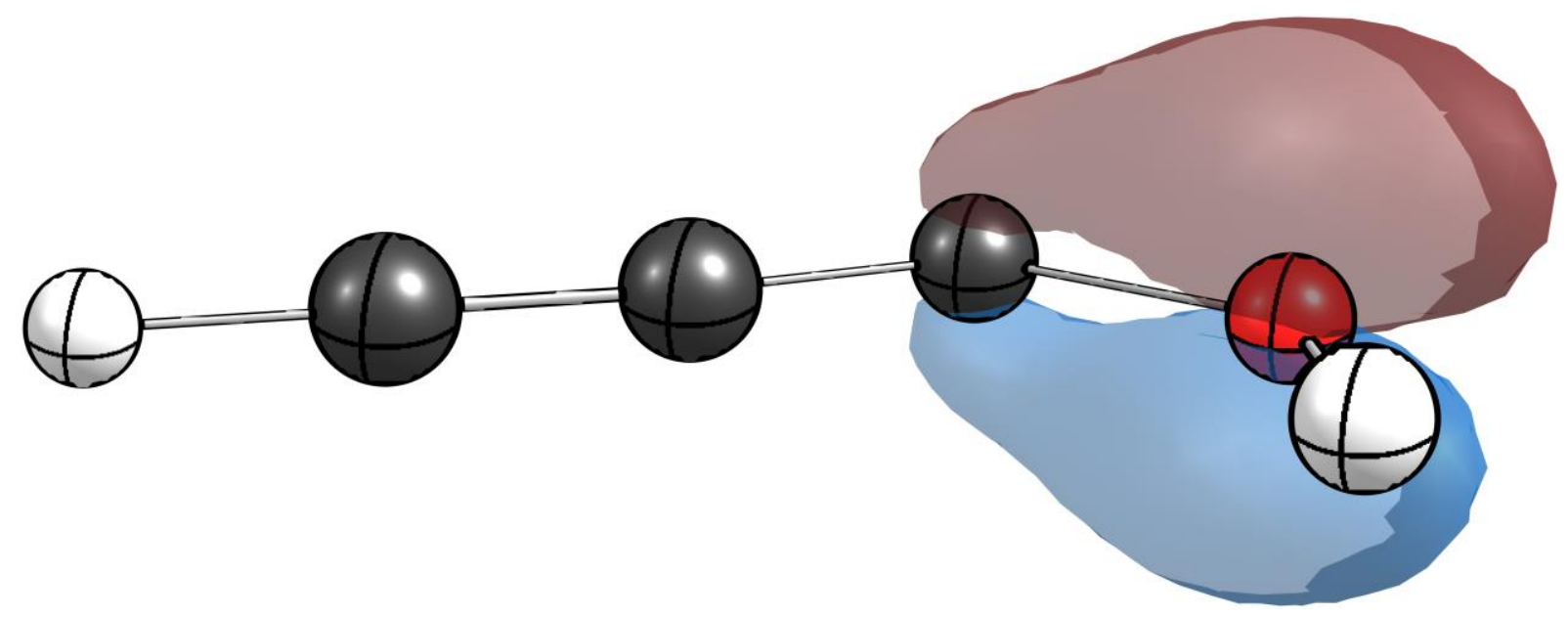

Figure S45: Depiction of the HOMO-3 of 1t. The NBO resembles a $\pi$ orbital along the C-O bond. It can be considered as the bonding combination of the oxygen electron lone pair with the empty p-orbital at the carbene center.

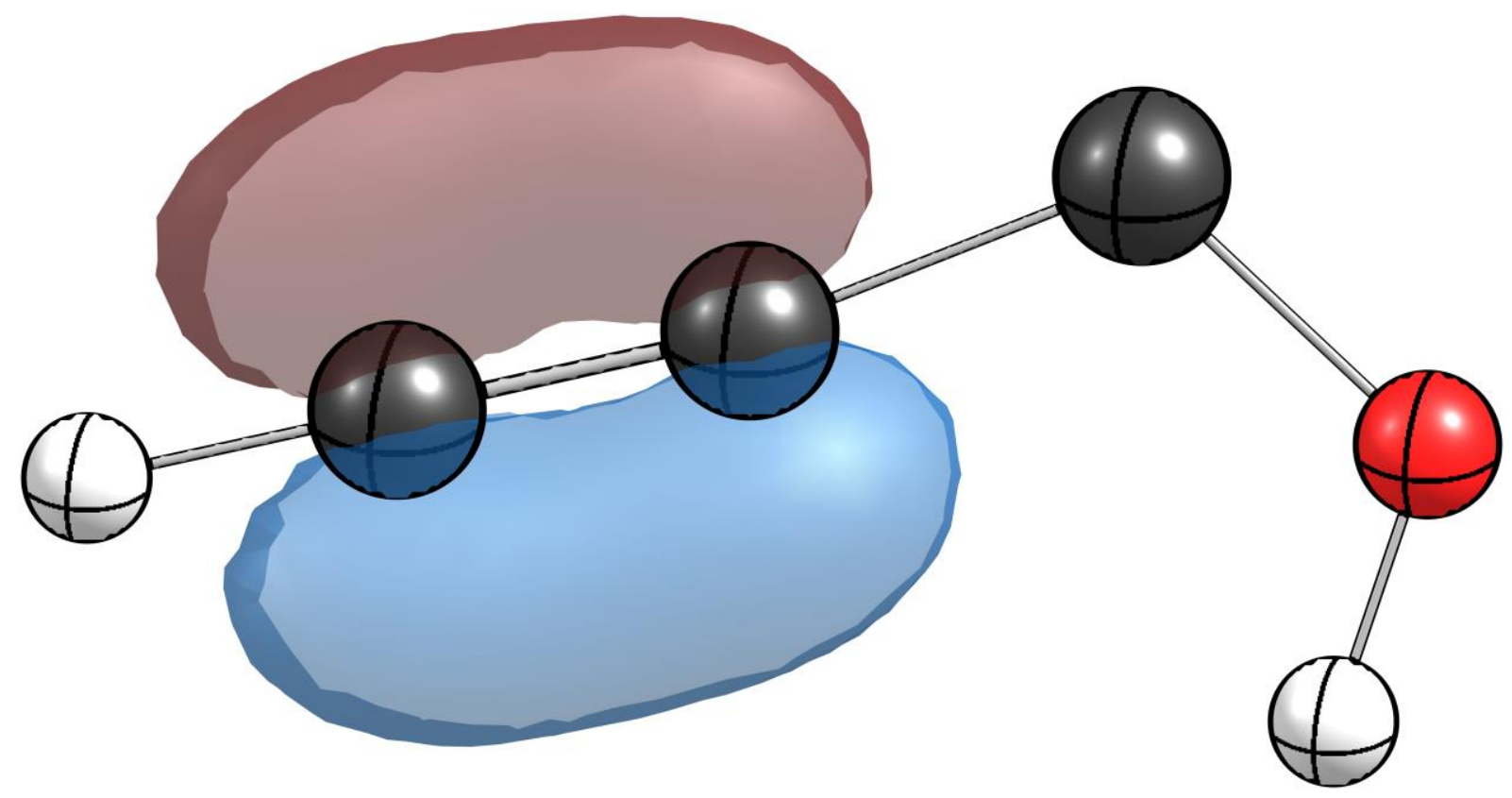

Figure S46: Depiction of the HOMO-2 of 1t. The NBO resembles the in-plane $\pi$-bond at the alkyne moiety. 


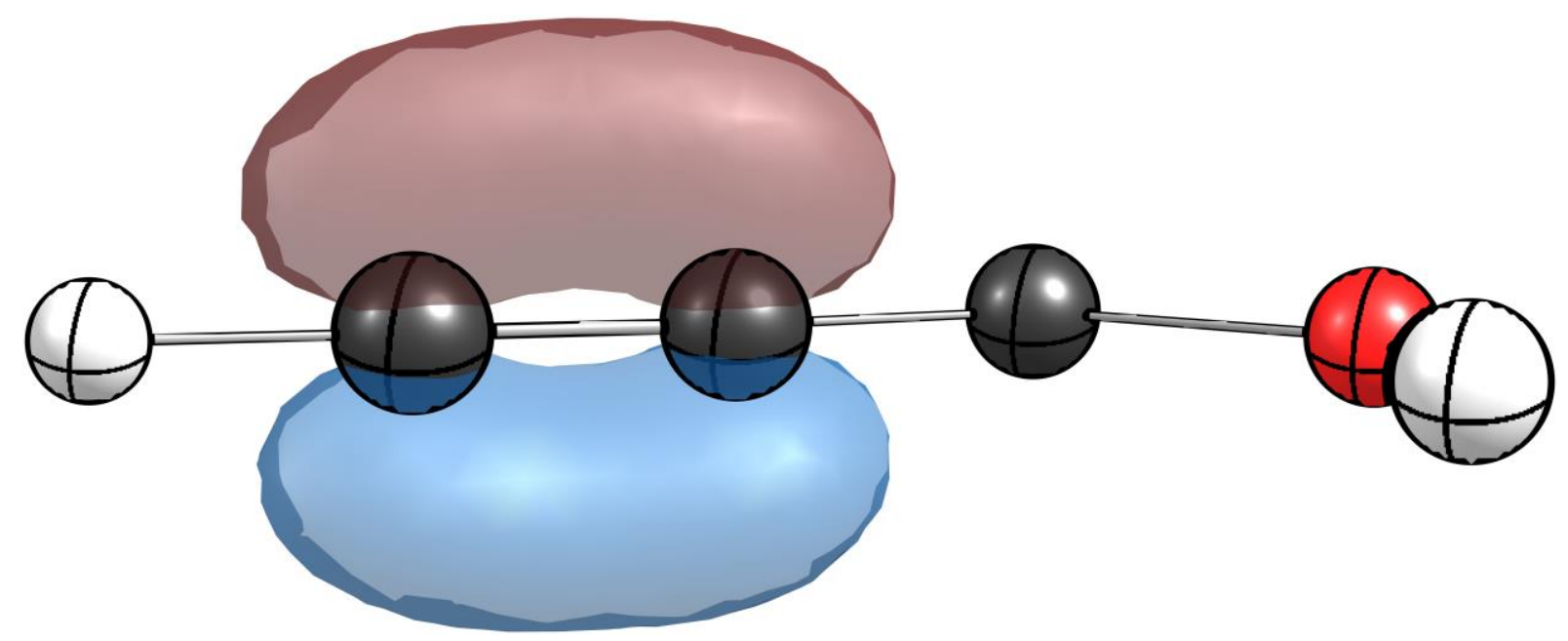

Figure S47: Depiction of the HOMO-1 of 1t. The NBO resembles the out-of-plane $\pi$-bond at the alkyne moiety.

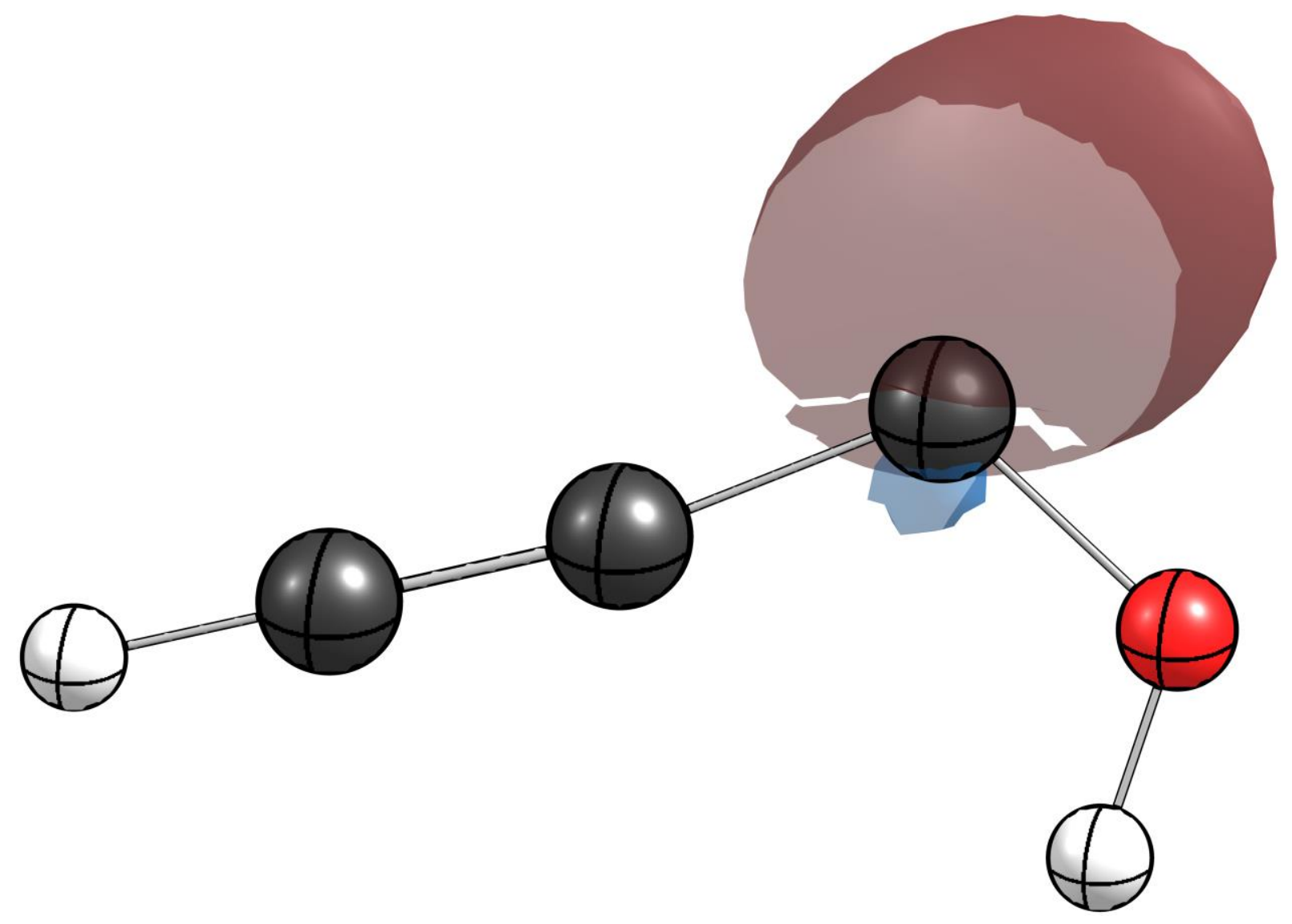

Figure S48: Depiction of the HOMO of 1t. The NBO resembles the electron lone pair at the carbene center. 


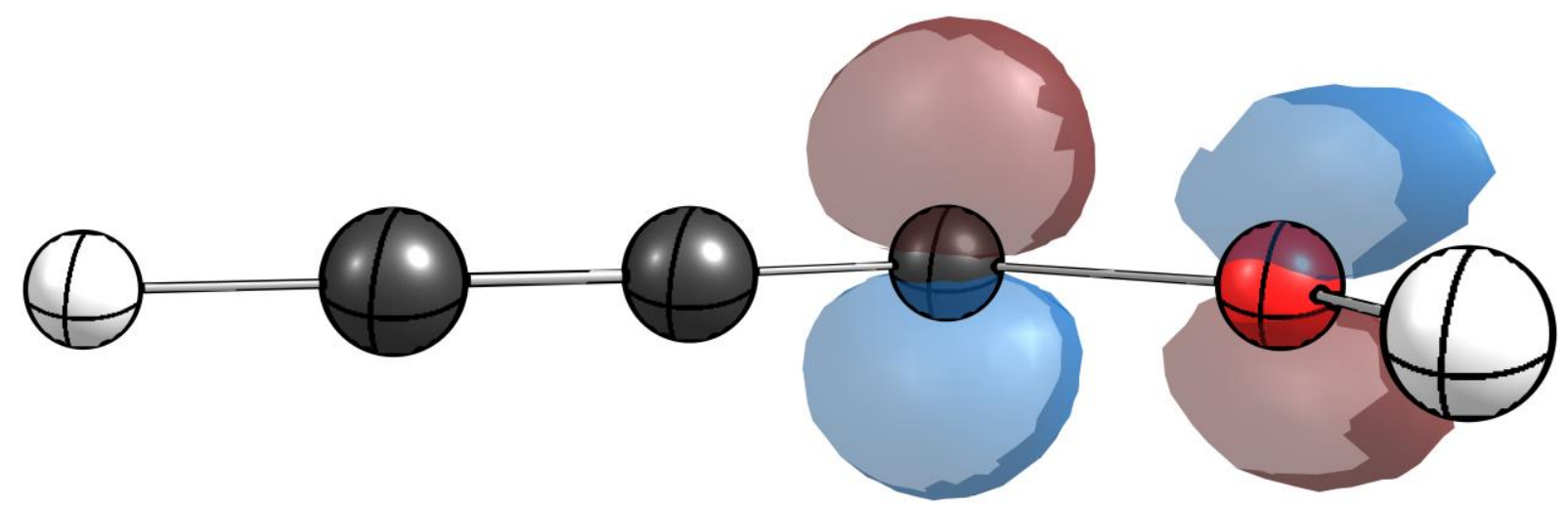

Figure S49: Depiction of the LUMO of 1t. The NBO resembles a $\pi^{*}$ orbital along the C-O bond. It can be considered as the antibonding combination of the oxygen electron lone pair with the empty p-orbital at the carbene center.

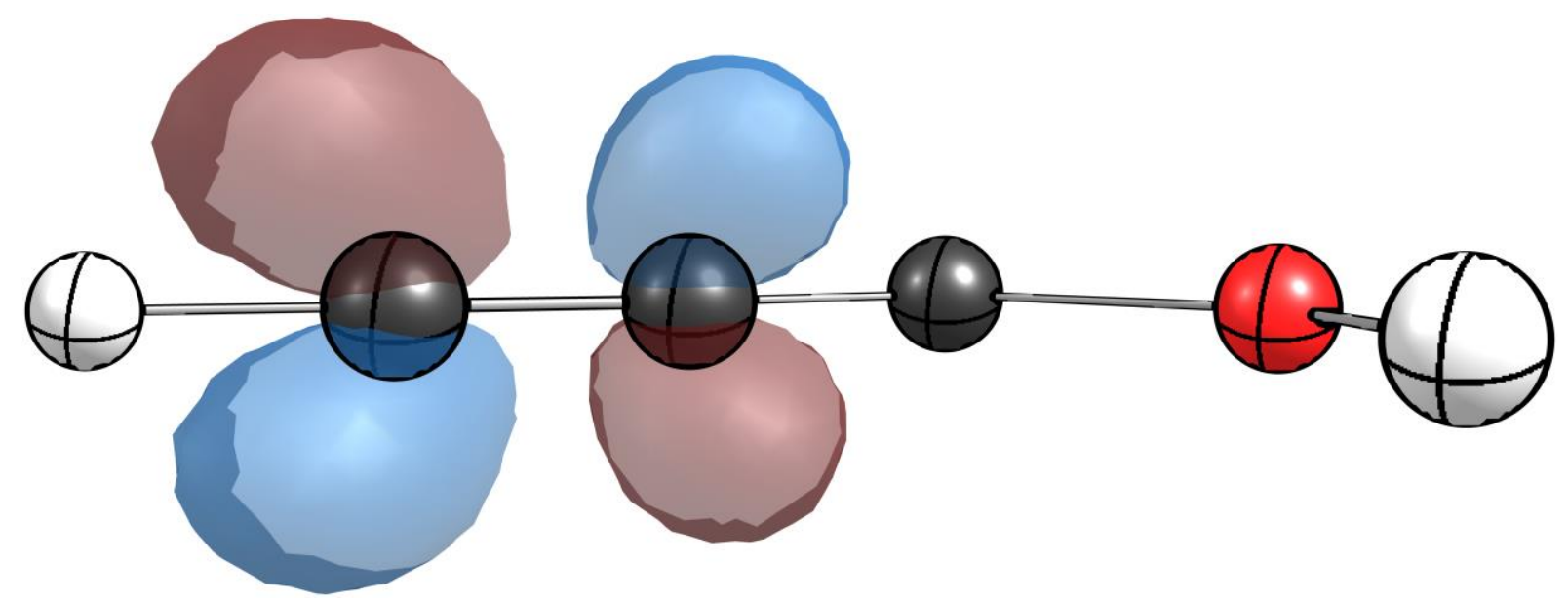

Figure S50: Depiction of the LUMO+1 of 1t. The NBO resembles the out-of-plane $\pi^{*}$ bond at the alkyne moiety. 


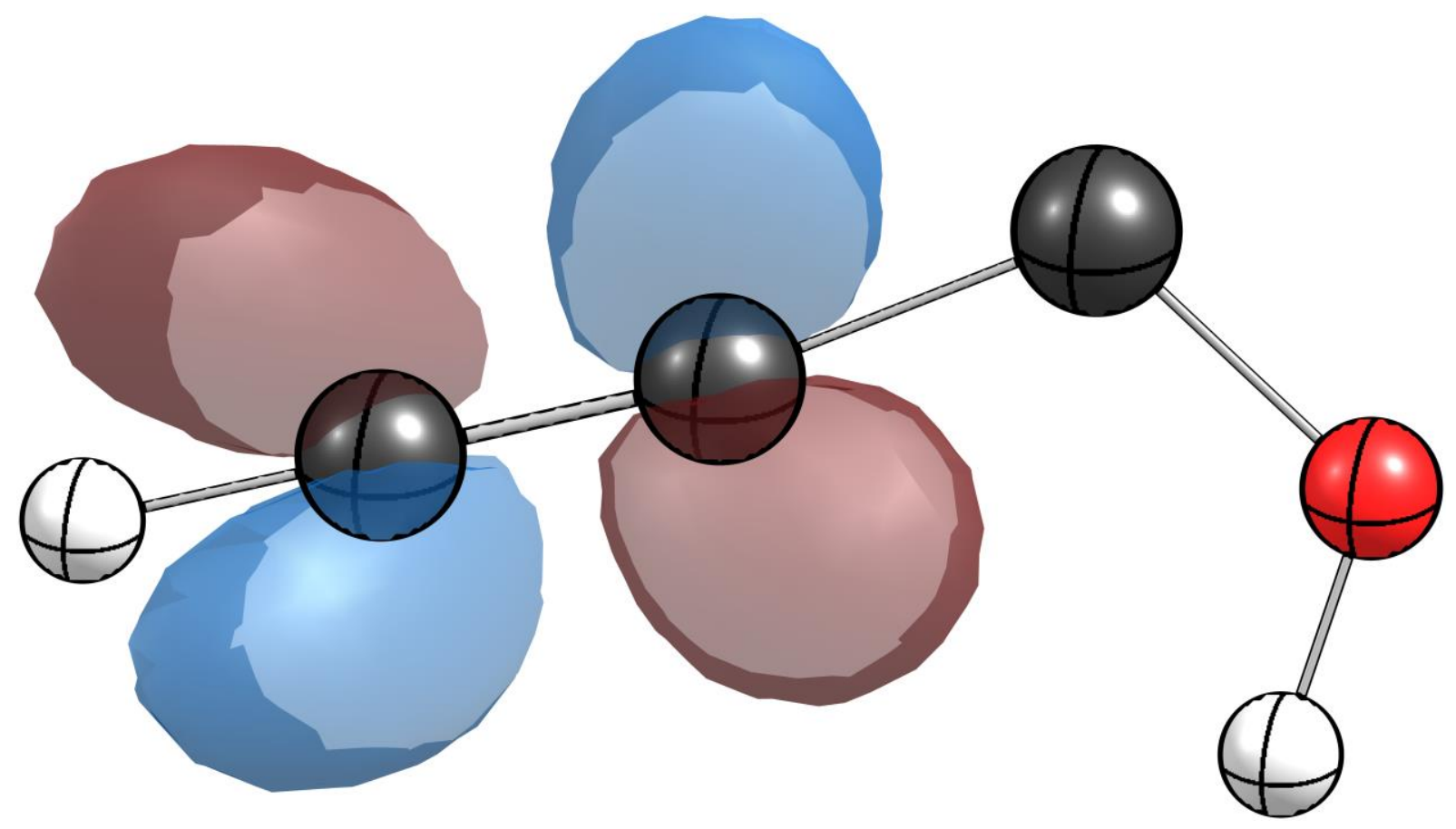

Figure S51: Depiction of the LUMO+2 of $1 \mathrm{t}$. The NBO resembles the in-plane alkyne $\pi^{*}$ bond at the alkyne moiety.

NRT Analysis:

Wiberg Bond Indices:<smiles>C#C[O-]</smiles>

$56.9 \%$<smiles>[C-]C=C[OH2+]</smiles>

$15.6 \%$<smiles>[CH-]=C=[C-]O</smiles>

$13.0 \%$<smiles></smiles>

0.93
$\mathrm{H}_{1}: 0.95$

$\mathrm{C}_{1}: 3.90$

$\mathrm{C}_{2}: 3.98$

$\mathrm{C}_{3}: 2.80$

$\mathrm{O}_{1}: 2.13$

$\mathrm{H}_{2}: 0.80$

Figure S52: Natural resonance theory (NRT) analysis of cis-ethynylhydroxycarbene 1c at B3LYP/def2QZVPP based on the optimized geometry of $\mathbf{1 c}$ at the same level of theory. Only the most relevant mesomeric structures contributing more than $5 \%$ are shown. 


\section{Triplet Ethynylhydroxycarbene}

All hydroxycarbenes we have investigated in our group display singlet ground states with adiabatic singlet-triplet energy gaps ranging from ca. 20 to $30 \mathrm{kcal} \mathrm{mol}^{-1}$. Considering the PES of rotamerizations in hydroxycarbenes, minima geometries on the singlet PES represent transition structures on the triplet PES and vice versa. This presumably is the reason why short UV irradiation of some transhydroxycarbenes $\left(\mathrm{F}_{3} \mathrm{C}-\ddot{\mathrm{C}}-\mathrm{OH},{ }^{20} \mathrm{NC}-\ddot{\mathrm{C}}-\mathrm{OH},{ }^{1}\right.$ and $\left.\mathbf{1}\right)$ generates the corresponding cis-conformers in minor yields as a photochemical equilibrium is readily reached.

However, when investigating the triplet PES of the rotamerization of $\mathbf{1}$, we only found one minimum structure $\mathbf{1}^{\mathbf{3}}$ (Figure S53), which is geometrically different from the transition structure between $\mathbf{1 t}$ and $\mathbf{1 c}$ on the singlet PES. The adiabatic singlet-triplet energy gap between $\mathbf{1 t}$ and ${ }^{\mathbf{3}} \mathbf{1}$ is only $12.6 \mathrm{kcal} \mathrm{mol}^{-1}$ at the B3LYP/def2-QZVPP level of theory. This is by far the smallest singlet-triplet energy gap for hydroxycarbenes reported so far, which can presumably be attributed to the unusual geometry of ${ }^{3} \mathbf{1}$.

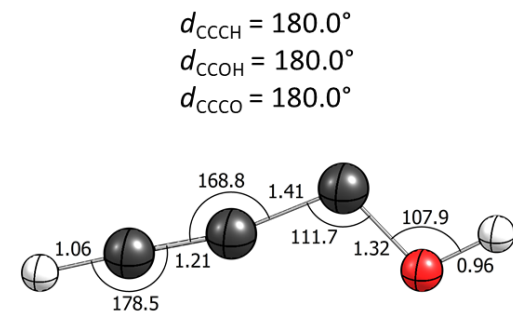

$1 \mathrm{t}$ 0.0

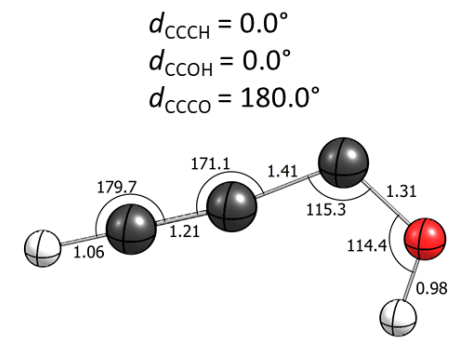

$1 c$

singlet

2.6

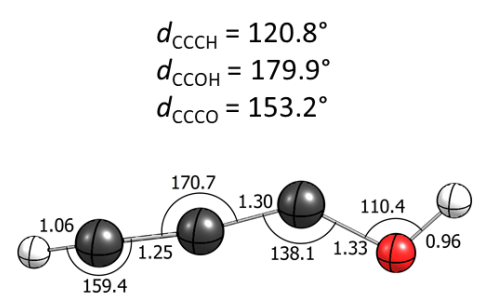

31

triplet

12.6

Figure S53: Comparison of the minimum geometries of singlet and triplet ethynylhydroxycarbene (1) optimized at the B3LYP/def2-QZVPP level of theory. Relative energies are given in $\mathrm{kcal} \mathrm{mol}^{-1}$ at the same level of theory including ZPVE corrections. The vertical singlet-triplet energy band of $\mathbf{1 t}$ is $25.4 \mathrm{kcal} \mathrm{mol}^{-1}$. Bond lengths are given in $\AA$ and angles in degree. Color code: Carbon - black, hydrogen - white, oxygen - red.

The bond angles in triplet ${ }^{3} \mathbf{1}$ are considerably wider than in the singlet conformers. This trend is even more pronounced comparing B3LYP/def2-QZVPP geometries of singlet and triplet propynylidene (29, $\mathrm{HCCCH}$, Figure S54). 
$d_{\mathrm{CCCH}}=180.0^{\circ}$

$d_{\mathrm{CCCH}}=-180.0^{\circ}$

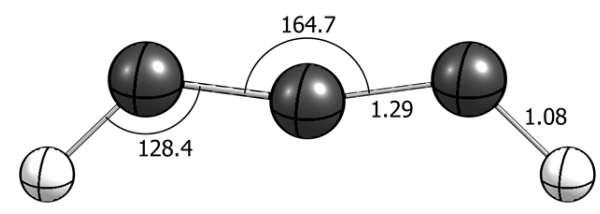

129

singlet

15.2
$d_{\mathrm{CCCH}}=180.0^{\circ}$

$d_{\mathrm{CCCH}}=180.0^{\circ}$

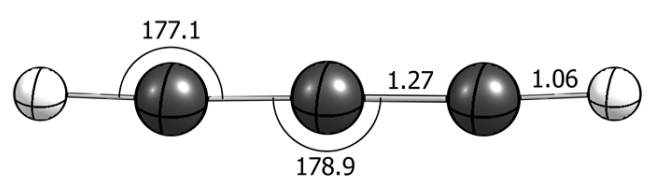

${ }^{3} 29$

triplet

0.0

B3LYP/def2-QZVPP

Figure S54: Comparison of the geometries of singlet and triplet propynylidene (29) optimized at the B3LP/def2-QZVPP level of theory. Relative energies are given in $\mathrm{kcal} \mathrm{mol}^{-1}$ at the same level of theory including ZPVE corrections. Bond lengths are given in $\AA$ and angles in degree. Color code: Carbon black, hydrogen - white. 


\section{Cartesian Coordinates of Computationally Optimized Geometries}

Optimized structures (Cartesian coordinates in Ångstrom), electronic energies, and zero-point vibrational energies (ZPVE) are provided. For transition states, the imaginary frequency is given additionally.

$B 3 L Y P /$ def2-QZVPP

\begin{tabular}{|c|c|c|c|}
\hline 6 & 0.351184000 & 1.850153000 & 0.000000000 \\
\hline 6 & 0.000000000 & 0.692596000 & 0.000000000 \\
\hline 1 & 0.685565000 & 2.857670000 & 0.000000000 \\
\hline 6 & -0.662408000 & -0.548692000 & 0.000000000 \\
\hline 8 & 0.187990000 & -1.555907000 & 0.000000000 \\
\hline 1 & -0.322136000 & -2.374755000 & 0.000000000 \\
\hline
\end{tabular}

cis-Ethynylhydroxycarbene 1c $\left(C_{s}\right)$

$\begin{array}{llll}6 & 0.468723000 & 1.792278000 & 0.000000000 \\ 6 & 0.000000000 & 0.676609000 & 0.000000000 \\ 1 & 0.874931000 & 2.773499000 & 0.000000000 \\ 6 & -0.739108000 & -0.522780000 & 0.000000000 \\ 8 & -0.024346000 & -1.621847000 & 0.000000000 \\ 1 & 0.942152000 & -1.475367000 & 0.000000000\end{array}$

$E=-190.6654691 \mathrm{au}$

ZPVE $=0.0361961 \mathrm{au}$

TS2: $1 \mathrm{c} \rightarrow \mathbf{1 t}\left(C_{1}\right)$

$\begin{array}{llll}6 & -1.877687000 & -0.158882000 & -0.002873000 \\ 6 & -0.686093000 & 0.077162000 & 0.002988000 \\ 1 & -2.915193000 & -0.386164000 & -0.009429000 \\ 6 & 0.599399000 & 0.630295000 & 0.049123000 \\ 8 & 1.596004000 & -0.278694000 & -0.121772000 \\ 1 & 1.933442000 & -0.675736000 & 0.688186000\end{array}$

$E=-190.6304663 \mathrm{au}$

ZPVE $=0.0333975 \mathrm{au}$

$v_{\mathrm{i}}=1121.2647 i \mathrm{~cm}^{-1}$ 
Triplet Ethynylhydroxycarbene ${ }^{3} 1\left(C_{1}\right)$

\begin{tabular}{lrrr}
6 & 1.974850000 & -0.084271000 & -0.065328000 \\
6 & 0.733976000 & 0.041542000 & 0.017101000 \\
1 & 2.985042000 & -0.214799000 & 0.241875000 \\
6 & -0.529851000 & 0.359636000 & 0.007846000 \\
8 & -1.704559000 & -0.258697000 & 0.000473000 \\
1 & -2.422417000 & 0.382932000 & -0.003370000 \\
\multicolumn{3}{c}{$\mathrm{E}=-190.6471122 \mathrm{au}$} & \\
\multicolumn{3}{l}{ ZPVE $=0.0337749 \mathrm{au}$}
\end{tabular}

Propynal $\mathbf{2}\left(C_{s}\right)$

$\begin{array}{llll}6 & -0.363195000 & 1.830876000 & 0.000000000\end{array}$

$\begin{array}{llll}6 & 0.000000000 & 0.686845000 & 0.000000000\end{array}$

$\begin{array}{llll}1 & -0.704253000 & 2.836841000 & 0.000000000\end{array}$

$\begin{array}{lllll}6 & 0.496375000 & -0.669840000 & 0.000000000\end{array}$

$\begin{array}{lllll}8 & -0.211397000 & -1.646152000 & 0.000000000\end{array}$

$\begin{array}{llll}1 & 1.596346000 & -0.754911000 & 0.000000000\end{array}$

$E=-190.7422812 \mathrm{au}$

ZPVE $=0.0370821 \mathrm{au}$

TS1: 1t $\rightarrow \mathbf{2}\left(C_{s}\right)$

$\begin{array}{rrrr}6 & -0.258436000 & 1.891917000 & 0.000000000 \\ 6 & 0.000000000 & 0.713236000 & 0.000000000 \\ 1 & -0.516087000 & 2.922228000 & 0.000000000 \\ 6 & 0.519313000 & -0.594265000 & 0.000000000 \\ 8 & -0.247762000 & -1.651450000 & 0.000000000 \\ 1 & 0.932927000 & -1.775957000 & 0.000000000\end{array}$

$E=-190.6129484 \mathrm{au}$

ZPVE $=0.0308291 \mathrm{au}$

$v_{\mathrm{i}}=2099.2476 i \mathrm{~cm}^{-1}$ 
trans-Ethynylglyoxylic acid $9 \mathrm{t}\left(C_{\mathrm{s}}\right)$

$\begin{array}{llll}6 & 2.640744000 & 0.614855000 & 0.000000000 \\ 6 & 1.441508000 & 0.608833000 & 0.000000000 \\ 1 & 3.703003000 & 0.617366000 & 0.000000000 \\ 6 & 0.000000000 & 0.648756000 & 0.000000000 \\ 8 & -0.638357000 & 1.668957000 & 0.000000000 \\ 6 & -0.680443000 & -0.740327000 & 0.000000000 \\ 8 & -0.067271000 & -1.770847000 & 0.000000000 \\ 8 & -2.011408000 & -0.633234000 & 0.000000000 \\ 1 & -2.377581000 & -1.529078000 & 0.000000000\end{array}$

$E=-379.4011494 \mathrm{au}$

ZPVE $=0.0523809 \mathrm{au}$

cis-Ethynylglyoxylic acid 9c $\left(C_{s}\right)$

$\begin{array}{llll}6 & 2.630173000 & 0.682243000 & 0.000000000 \\ 6 & 1.431403000 & 0.644917000 & 0.000000000 \\ 1 & 3.692277000 & 0.714219000 & 0.000000000 \\ 6 & 0.000000000 & 0.602479000 & 0.000000000 \\ 8 & -0.712087000 & 1.583503000 & 0.000000000 \\ 6 & -0.657956000 & -0.801216000 & 0.000000000 \\ 8 & -0.035120000 & -1.820233000 & 0.000000000 \\ 8 & -1.988103000 & -0.725924000 & 0.000000000 \\ 1 & -2.231516000 & 0.216479000 & 0.000000000\end{array}$

$E=-379.4047966 \mathrm{au}$

ZPVE $=0.0527462 \mathrm{au}$

TS3: 9t $\rightarrow$ 9c $\left(C_{1}\right)$

$\begin{array}{lrrr}6 & -2.667331000 & -0.475084000 & 0.212981000 \\ 6 & -1.574292000 & -0.003972000 & 0.064611000 \\ 1 & -3.637086000 & -0.889980000 & 0.339588000 \\ 6 & -0.261594000 & 0.570896000 & -0.070267000 \\ 8 & -0.055707000 & 1.741166000 & -0.277877000 \\ 6 & 0.919879000 & -0.425927000 & -0.013438000 \\ 8 & 0.807066000 & -1.556440000 & -0.371230000 \\ 8 & 2.060674000 & 0.117834000 & 0.485670000 \\ 1 & 2.640845000 & 0.474009000 & -0.195409000\end{array}$

$E=-379.3839314 \mathrm{au}$

ZPVE $=0.050556 \mathrm{au}$

$v_{\mathrm{i}}=622.042 i \mathrm{~cm}^{-1}$ 


\begin{tabular}{lrrr}
\multicolumn{5}{c}{ trans'-Ethynylglyoxylic acid $9 \mathbf{t t}^{\prime}\left(C_{s}\right)$} \\
6 & 2.625735000 & 0.406722000 & 0.000000000 \\
6 & 1.431416000 & 0.519734000 & 0.000000000 \\
1 & 3.683518000 & 0.309149000 & 0.000000000 \\
6 & 0.000000000 & 0.689427000 & 0.000000000 \\
8 & -0.535896000 & 1.765775000 & 0.000000000 \\
6 & -0.880813000 & -0.589767000 & 0.000000000 \\
8 & -2.073685000 & -0.554935000 & 0.000000000 \\
8 & -0.139907000 & -1.710810000 & 0.000000000 \\
1 & -0.745639000 & -2.466083000 & 0.000000000
\end{tabular}

$E=-379.3995571 \mathrm{au}$

ZPVE $=0.0522909 \mathrm{au}$

cis'-Ethynylglyoxylic acid $9 \mathbf{c}^{\prime}\left(C_{s}\right)$

$\begin{array}{llll}6 & 2.607247000 & 0.213435000 & 0.000000000 \\ 6 & 1.430447000 & 0.454167000 & 0.000000000 \\ 1 & 3.654533000 & 0.030220000 & 0.000000000 \\ 6 & 0.000000000 & 0.699295000 & 0.000000000 \\ 8 & -0.474738000 & 1.800362000 & 0.000000000 \\ 6 & -0.924561000 & -0.560457000 & 0.000000000 \\ 8 & -2.109220000 & -0.459671000 & 0.000000000 \\ 8 & -0.291299000 & -1.746212000 & 0.000000000 \\ 1 & 0.668728000 & -1.624688000 & 0.000000000\end{array}$

$E=-379.3949404 \mathrm{au}$

ZPVE $=0.0521899 \mathrm{au}$

trans-3-Hydroxypropadienylidene $\mathbf{5 t}\left(C_{s}\right)$

$\begin{array}{lrrr}6 & -1.304390000 & -1.564636000 & 0.000000000 \\ 6 & -0.654423000 & -0.480660000 & 0.000000000 \\ 6 & 0.000000000 & 0.684982000 & 0.000000000 \\ 8 & 1.314784000 & 0.820872000 & 0.000000000 \\ 1 & 1.730160000 & -0.055067000 & 0.000000000 \\ 1 & -0.495556000 & 1.649975000 & 0.000000000\end{array}$

$E=-190.6729163 \mathrm{au}$

ZPVE $=0.0381435 \mathrm{au}$ 
cis-3-Hydroxypropadienylidene $5 c\left(C_{s}\right)$

$\begin{array}{rrrr}6 & -1.318948000 & -1.607435000 & 0.000000000 \\ 6 & -0.612907000 & -0.557913000 & 0.000000000 \\ 6 & 0.000000000 & 0.625304000 & 0.000000000 \\ 8 & 1.321402000 & 0.750373000 & 0.000000000 \\ 1 & 1.581112000 & 1.679450000 & 0.000000000 \\ 1 & -0.561197000 & 1.557832000 & 0.000000000\end{array}$

$E=-190.6662018 \mathrm{au}$

ZPVE $=0.0378155 \mathrm{au}$

TS4: $5 c \rightarrow 5 t\left(C_{1}\right)$

$\begin{array}{lrrr}6 & -2.052282000 & -0.255715000 & 0.011391000 \\ 6 & -0.821156000 & 0.060685000 & -0.012068000 \\ 6 & 0.437493000 & 0.482894000 & 0.018166000 \\ 8 & 1.508681000 & -0.329303000 & -0.114314000 \\ 1 & 1.868681000 & -0.635994000 & 0.725617000 \\ 1 & 0.677536000 & 1.543234000 & 0.083963000\end{array}$

$E=-190.6486585 \mathrm{au}$

ZPVE $=0.0357238 \mathrm{au}$

$v_{\mathrm{i}}=728.9791 \mathrm{~cm}^{-1}$

Ethynylglyoxylic acid ethyl ester $\mathbf{1 0}\left(C_{s}\right)$

$\begin{array}{lrrr}6 & -2.516516000 & 0.338036000 & 0.000000000 \\ 6 & -1.308055000 & -0.452508000 & 0.000000000 \\ 8 & -1.308211000 & -1.656499000 & 0.000000000 \\ 6 & -3.543471000 & 0.957562000 & 0.000000000 \\ 1 & -4.450679000 & 1.509842000 & 0.000000000 \\ 6 & 0.000000000 & 0.375939000 & 0.000000000 \\ 8 & 1.065330000 & -0.410860000 & 0.000000000 \\ 8 & 0.002148000 & 1.578465000 & 0.000000000 \\ 6 & 2.357463000 & 0.250233000 & 0.000000000 \\ 6 & 3.421094000 & -0.821749000 & 0.000000000 \\ 1 & 2.413436000 & 0.888826000 & 0.880602000 \\ 1 & 2.413436000 & 0.888826000 & -0.880602000 \\ 1 & 3.340194000 & -1.452874000 & 0.883403000 \\ 1 & 4.406194000 & -0.355678000 & 0.000000000 \\ 1 & 3.340194000 & -1.452874000 & -0.883403000 \\ & & & \\ \text { E }=-458.0572755 \mathrm{au} & & \\ \text { ZPVE = } 0.108356 \mathrm{au} & & \end{array}$


trans-Ethynylhydroxycarbene $\cdot \mathrm{CO}_{2}$ complex $\mathbf{1 t} \cdot \mathrm{CO}_{\mathbf{2}}\left(C_{\mathrm{s}}\right)$

$\begin{array}{lrrr}6 & 1.398891000 & 3.059816000 & 0.000000000 \\ 6 & 0.648981000 & 2.111394000 & 0.000000000 \\ 1 & 2.034497000 & 3.910153000 & 0.000000000 \\ 6 & 0.000000000 & 0.863456000 & 0.000000000 \\ 8 & -1.305505000 & 0.973530000 & 0.000000000 \\ 6 & -0.088262000 & -2.152948000 & 0.000000000 \\ 8 & 1.015231000 & -2.489994000 & 0.000000000 \\ 8 & -1.224855000 & -1.892817000 & 0.000000000 \\ 1 & -1.671124000 & 0.073789000 & 0.000000000\end{array}$

$E=-379.3571034 \mathrm{au}$

ZPVE $=0.0498309 \mathrm{au}$

$\begin{array}{lrrr}\text { TS5: } 10 \rightarrow 9 \mathrm{c}\left(C_{1}\right) & & \\ 6 & -2.364105000 & -0.438815000 & 0.071195000 \\ 6 & -1.369486000 & 0.610226000 & 0.043955000 \\ 8 & -1.636870000 & 1.770842000 & 0.222695000 \\ 6 & -3.212408000 & -1.287039000 & 0.094499000 \\ 1 & -3.962382000 & -2.038558000 & 0.114910000 \\ 6 & 0.094415000 & 0.157799000 & -0.241535000 \\ 8 & 0.960614000 & 1.049486000 & -0.325616000 \\ 8 & 0.302054000 & -1.085998000 & -0.347238000 \\ 6 & 2.862351000 & 0.376725000 & 0.137637000 \\ 6 & 2.843052000 & -1.007197000 & 0.305897000 \\ 1 & 2.801201000 & 1.051168000 & 0.977184000 \\ 1 & 3.190484000 & 0.822277000 & -0.787688000 \\ 1 & 3.349105000 & -1.605044000 & -0.444820000 \\ 1 & 1.605635000 & -1.182536000 & -0.046000000 \\ 1 & 2.886666000 & -1.392150000 & 1.317790000 \\ & & & \\ E= & -457.9810044 \text { au } & & \\ \text { ZPVE }=0.101181 \mathrm{au} & & \\ V_{\mathrm{i}}= & 1297.1681 i \mathrm{~cm}^{-1} & & \end{array}$


TS6: $9 \mathrm{c} \rightarrow 1 \mathrm{t} \cdot \mathrm{CO}_{2}\left(C_{s}\right)$

$\begin{array}{lrrr}6 & 2.149303000 & 2.086664000 & 0.000000000 \\ 6 & 1.086804000 & 1.512372000 & 0.000000000 \\ 1 & 3.068369000 & 2.619372000 & 0.000000000 \\ 6 & 0.000000000 & 0.635093000 & 0.000000000 \\ 8 & -1.174206000 & 1.161752000 & 0.000000000 \\ 6 & -0.447575000 & -1.554229000 & 0.000000000 \\ 8 & 0.551271000 & -2.160727000 & 0.000000000 \\ 8 & -1.627387000 & -1.387319000 & 0.000000000 \\ 1 & -1.796985000 & 0.391580000 & 0.000000000\end{array}$

$E=-379.3536976$ au ZPVE $=0.0499679 \mathrm{au}$ $v_{\mathrm{i}}=166.4588 \mathrm{i} \mathrm{cm}^{-1}$

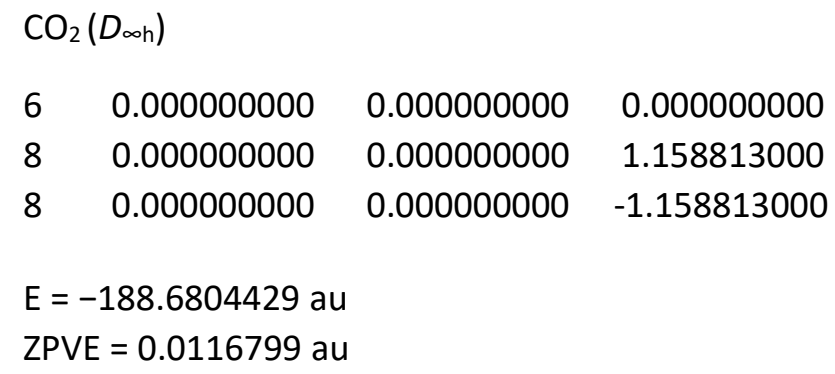




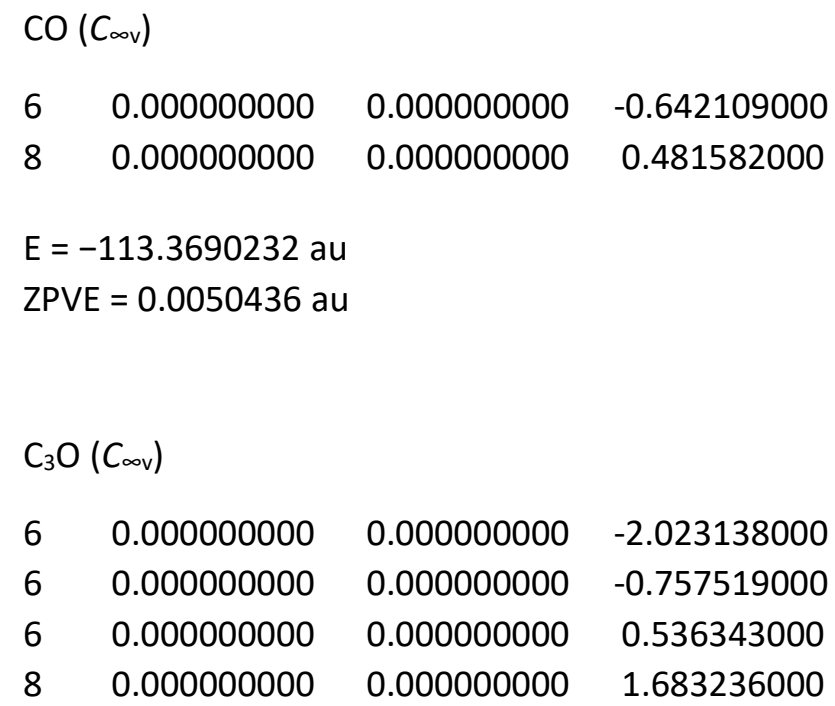




\begin{tabular}{lccc}
\multicolumn{5}{c}{ Propadienone $4\left(C_{S}\right)$} \\
6 & 0.915355000 & 1.579964000 & 0.000000000 \\
6 & 0.000000000 & 0.629758000 & 0.000000000 \\
1 & 0.642876000 & 2.630540000 & 0.000000000 \\
6 & -0.314259000 & -0.621547000 & 0.000000000 \\
8 & -0.778896000 & -1.690986000 & 0.000000000 \\
1 & 1.981714000 & 1.368302000 & 0.000000000 \\
& & & \\
$E$ & & $-190.7511017 \mathrm{au}$ &
\end{tabular}

Cyclopropenone $\mathbf{3}\left(C_{2 v}\right)$

$\begin{array}{llll}6 & -0.000001000 & -0.927262000 & 0.669400000 \\ 6 & -0.000001000 & -0.927262000 & -0.669400000 \\ 6 & -0.000001000 & 0.332133000 & 0.000000000 \\ 8 & 0.000001000 & 1.531866000 & 0.000000000 \\ 1 & 0.000003000 & -1.560293000 & 1.544510000 \\ 1 & 0.000003000 & -1.560293000 & -1.544510000\end{array}$

$E=-190.7344703 \mathrm{au}$

ZPVE $=0.037601 \mathrm{au}$

$6\left(C_{s}\right)$

$\begin{array}{llll}6 & -1.260641000 & -1.679731000 & 0.000000000 \\ 6 & -0.515337000 & -0.633243000 & 0.000000000 \\ 6 & 0.000000000 & 0.553217000 & 0.000000000 \\ 8 & 1.187857000 & 1.065792000 & 0.000000000 \\ 1 & 1.153020000 & 2.032204000 & 0.000000000\end{array}$

$E=-190.0063224 \mathrm{au}$

ZPVE $=0.0249328 \mathrm{au}$

\section{$7\left(C_{1}\right)$}

$\begin{array}{llll}6 & 1.047172000 & -0.411062000 & -0.022875000\end{array}$

$\begin{array}{llll}6 & -0.422508000 & -0.661121000 & 0.036495000\end{array}$

$\begin{array}{llll}8 & -1.290431000 & 0.318050000 & -0.025275000\end{array}$

$\begin{array}{llll}6 & 1.176970000 & 0.874550000 & 0.019692000\end{array}$

$\begin{array}{lllll}1 & -2.169803000 & -0.078201000 & 0.043513000\end{array}$

$\begin{array}{lllll}1 & 1.683452000 & -1.280399000 & -0.041186000\end{array}$

$E=-190.5973597 \mathrm{au}$

ZPVE $=0.0350823 \mathrm{au}$ 
$8\left(C_{s}\right)$

$\begin{array}{rrrr}6 & 0.112935000 & -1.633736000 & 0.000000000 \\ 6 & 0.853401000 & -0.470733000 & 0.000000000 \\ 1 & -0.970988000 & -1.460427000 & 0.000000000 \\ 6 & 0.000000000 & 0.588059000 & 0.000000000 \\ 8 & -0.844674000 & 1.366636000 & 0.000000000 \\ 1 & 1.930362000 & -0.374203000 & 0.000000000\end{array}$

$E=-190.6988502 \mathrm{au}$

ZPVE $=0.0365366 \mathrm{au}$

Hydrogen atom

$E=-0.5024062 \mathrm{au}$

$\mathrm{H}_{2}\left(D_{\infty \mathrm{h}}\right)$

$\begin{array}{llll}1 & 0.000000000 & 0.000000000 & 0.370912000\end{array}$

$\begin{array}{llll}1 & 0.000000000 & 0.000000000 & -0.370912000\end{array}$

$E=-1.1806347 \mathrm{au}$

ZPVE $=0.010061 \mathrm{au}$

$27\left(C_{s}\right)$

$\begin{array}{llll}6 & 0.978830000 & 1.143391000 & 0.000000000\end{array}$

$\begin{array}{llll}6 & 0.000000000 & 0.271314000 & 0.000000000\end{array}$

$\begin{array}{lllll}6 & -1.256743000 & -0.405478000 & 0.000000000\end{array}$

$\begin{array}{llll}8 & -0.136687000 & -1.136582000 & 0.000000000\end{array}$

$\begin{array}{llll}1 & 0.745333000 & 2.197497000 & 0.000000000\end{array}$

$\begin{array}{llll}1 & 2.015640000 & 0.839792000 & 0.000000000\end{array}$

$E=-190.6590987 \mathrm{au}$

ZPVE $=0.0363519 \mathrm{au}$

$28\left(C_{s}\right)$

$\begin{array}{lrrr}6 & 0.000000000 & 0.127056000 & 0.000000000 \\ 6 & 0.562845000 & -1.068253000 & 0.000000000 \\ 1 & 1.418939000 & -1.721598000 & 0.000000000 \\ 8 & 0.129157000 & 1.434121000 & 0.000000000 \\ 1 & -0.750298000 & 1.838908000 & 0.000000000 \\ 6 & -0.846495000 & -0.990515000 & 0.000000000\end{array}$

$E=-190.682288 a u$

ZPVE $=0.0383089 \mathrm{au}$ 
TS7: $\mathbf{2 7} \rightarrow \mathbf{4}\left(C_{S}\right)$

$\begin{array}{rrrr}6 & 1.648291000 & -0.167351000 & 0.000000000 \\ 6 & 0.431209000 & 0.252180000 & -0.000001000 \\ 1 & 2.460534000 & 0.549997000 & 0.000001000 \\ 6 & -0.897841000 & 0.686949000 & 0.000000000 \\ 8 & -1.427966000 & -0.494311000 & 0.000000000 \\ 1 & 1.873245000 & -1.226174000 & 0.000000000\end{array}$

$E=-190.6121594 \mathrm{au}$

ZPVE $=0.0326661 \mathrm{au}$

$v_{\mathrm{i}}=999.0922 i \mathrm{~cm}^{-1}$

TS8: $\mathbf{1 t} \rightarrow \mathbf{2 8}\left(C_{1}\right)$

$\begin{array}{rrrr}6 & 0.955223000 & -0.633732000 & 0.059543000 \\ 6 & 1.274795000 & 0.566651000 & -0.175131000 \\ 1 & 0.749710000 & -1.672500000 & 0.193004000 \\ 6 & -0.282215000 & 0.569132000 & 0.260206000 \\ 8 & -1.291557000 & -0.200535000 & -0.144248000 \\ 1 & -2.104075000 & 0.264472000 & 0.093273000\end{array}$

$E=-190.5765686 \mathrm{au}$

ZPVE $=0.0339473 \mathrm{au}$

$v_{\mathrm{i}}=671.1922 i \mathrm{~cm}^{-1}$

TS9: $1 \mathrm{c} \rightarrow \mathbf{4}\left(C_{s}\right)$

$\begin{array}{rrrr}6 & 1.239897000 & 0.631568000 & 0.000000000 \\ 6 & 0.000000000 & 0.922774000 & 0.000000000 \\ 1 & 2.233027000 & 1.045657000 & 0.000000000 \\ 6 & -0.950078000 & -0.001966000 & 0.000000000 \\ 8 & -0.590960000 & -1.186490000 & 0.000000000 \\ 1 & 0.755732000 & -0.867994000 & 0.000000000\end{array}$

$E=-190.5874509 \mathrm{au}$

ZPVE $=0.0314974 \mathrm{au}$

$v_{\mathrm{i}}=1747.0587 i \mathrm{~cm}^{-1}$ 


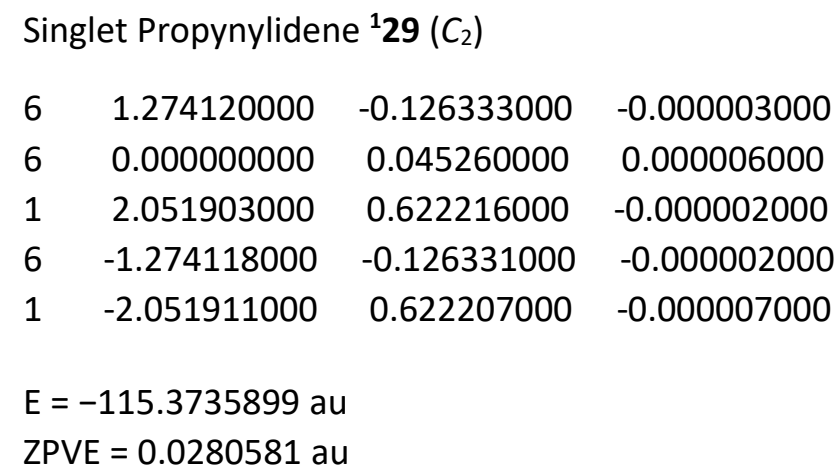

$\operatorname{CCSD}(T) / c c-p V T Z$

trans-Ethynylhydroxycarbene $\mathbf{1 t}\left(C_{s}\right)$

$\begin{array}{lrrr}6 & -1.895427915 & 0.161012259 & 0.000000000 \\ 6 & -0.698807265 & -0.073969359 & 0.000000000 \\ 1 & -2.928249341 & 0.415768389 & 0.000000000 \\ 6 & 0.631899581 & -0.619596792 & 0.000000000 \\ 8 & 1.506709590 & 0.375509933 & 0.000000000 \\ 1 & 2.380868595 & -0.034355589 & 0.000000000\end{array}$

$E=-190,2639917 \mathrm{au}$

ZPVE $=23.0123 \mathrm{kcal} \mathrm{mol}^{-1}$

cis-Ethynylhydroxycarbene $1 \mathrm{c}\left(C_{s}\right)$

$\begin{array}{lrrr}6 & -1.880610841 & 0.179768613 & 0.000000000 \\ 6 & -0.695206981 & -0.108515860 & 0.000000000 \\ 1 & -2.912396868 & 0.440556459 & 0.000000000 \\ 6 & 0.647468991 & -0.636636299 & 0.000000000 \\ 8 & 1.556923320 & 0.320044612 & 0.000000000 \\ 1 & 1.163413089 & 1.212028328 & 0.000000000\end{array}$

$E=-190.259751143774764$ au

ZPVE $=22.6880 \mathrm{kcal} \mathrm{mol}^{-1}$ 
TS2: 1c $\rightarrow$ 1t $\left(C_{1}\right)$

$\begin{array}{lrrr}1 & -2.936661073 & 0.441118091 & 0.003331726 \\ 6 & -1.905464290 & 0.177371244 & -0.000112090 \\ 6 & -0.705888017 & -0.066476675 & 0.000603759 \\ 6 & 0.606546064 & -0.639493190 & -0.021578208 \\ 8 & 1.572693289 & 0.328773295 & 0.066340988 \\ 1 & 1.847761782 & 0.634944292 & -0.805137591 \\ & & \\ E & =-190.222125617662215 \text { au } \\ Z\end{array}$

Propynal $\mathbf{2}\left(C_{s}\right)$

$\begin{array}{lrrr}6 & -1.910058390 & 0.159137431 & 0.000000000 \\ 6 & -0.723071418 & -0.091981140 & 0.000000000 \\ 1 & -2.945735421 & 0.403520153 & 0.000000000 \\ 6 & 0.689286536 & -0.453462277 & 0.000000000 \\ 8 & 1.587969832 & 0.361168584 & 0.000000000 \\ 1 & 0.888510647 & -1.535848481 & 0.000000000\end{array}$

$E=-190,3381968 \mathrm{au}$

ZPVE $=23.0591 \mathrm{kcal} \mathrm{mol}^{-1}$

TS1: 1t $\rightarrow \mathbf{2}\left(C_{S}\right)$

$\begin{array}{lrrr}6 & -1.935845219 & 0.140075186 & 0.000000000 \\ 6 & -0.735471618 & -0.062733730 & 0.000000000 \\ 1 & -2.975305570 & 0.367816686 & 0.000000000 \\ 6 & 0.624313105 & -0.515187147 & 0.000000000 \\ 8 & 1.607533784 & 0.356625153 & 0.000000000 \\ 1 & 1.835900602 & -0.814362992 & 0.000000000 \\ & & \\ E=-190,2071271 \mathrm{au} & \\ Z & \\ V_{i}=2137.8814 \mathrm{~cm}^{-1} & \end{array}$




\begin{tabular}{|c|c|c|c|}
\hline 6 & 1.998921242 & 0.242220466 & 0.000000000 \\
\hline 6 & 0.769983134 & -0.114996201 & 0.000000000 \\
\hline 6 & -0.516099088 & -0.509821072 & 0.000000000 \\
\hline 8 & -1.561046325 & 0.307993081 & 0.000000000 \\
\hline 1 & -0.824299935 & -1.550048895 & -0.000000005 \\
\hline 1 & -1.224528675 & 1.217489786 & -0.000000005 \\
\hline \multicolumn{4}{|c|}{$\begin{array}{l}E=-190.265116319612360 \mathrm{au} \\
Z P V E=23.9141 \mathrm{kcal} \mathrm{mol}^{-1}\end{array}$} \\
\hline
\end{tabular}

cis-3-Hydroxypropadienylidene $\mathbf{5 c}\left(C_{s}\right)$

$\begin{array}{llll}6 & 2.029460632 & 0.201110189 & 0.000000000 \\ 6 & 0.774331188 & -0.054935885 & 0.000000000 \\ 6 & -0.497748645 & -0.479879827 & 0.000000000 \\ 8 & -1.534940244 & 0.356925609 & 0.000000000 \\ 1 & -0.741851569 & -1.540697724 & 0.000000000 \\ 1 & -2.355193937 & -0.150596193 & 0.000000000\end{array}$

$E=-190.257773178081749 \mathrm{au}$

ZPVE $=23.6963 \mathrm{kcal} \mathrm{mol}^{-1}$

TS4: $5 c \rightarrow 5 t\left(C_{1}\right)$

$\begin{array}{lrrr}6 & 2.022914382 & 0.240293668 & 0.010368487 \\ 6 & 0.775931764 & -0.082882360 & -0.016761965 \\ 6 & -0.492695415 & -0.506495463 & 0.018092263 \\ 8 & -1.566293313 & 0.324754490 & -0.063398334 \\ 1 & -0.731130073 & -1.568527690 & 0.049199604 \\ 1 & -1.869600070 & 0.570923518 & 0.817682494\end{array}$

$E=-190.241341397177081 \mathrm{au}$

ZPVE $=22.4518 \mathrm{kcal} \mathrm{mol}^{-1}$

$V_{\mathrm{i}}=680.51383 i \mathrm{~cm}^{-1}$

Acetylene $\left(D_{\infty h}\right)$

$\begin{array}{llll}6 & 0.000000000 & 0.000000000 & 0.604849897\end{array}$

$\begin{array}{llll}6 & 0.000000000 & 0.000000000 & -0.604849897\end{array}$

$\begin{array}{lllll}1 & 0.000000000 & 0.000000000 & -1.668544667\end{array}$

$\begin{array}{llll}1 & 0.000000000 & 0.000000000 & 1.668544667\end{array}$

$E=-77.187648308229456 \mathrm{au}$

ZPVE $=16.5395 \mathrm{kcal} \mathrm{mol}^{-1}$ 


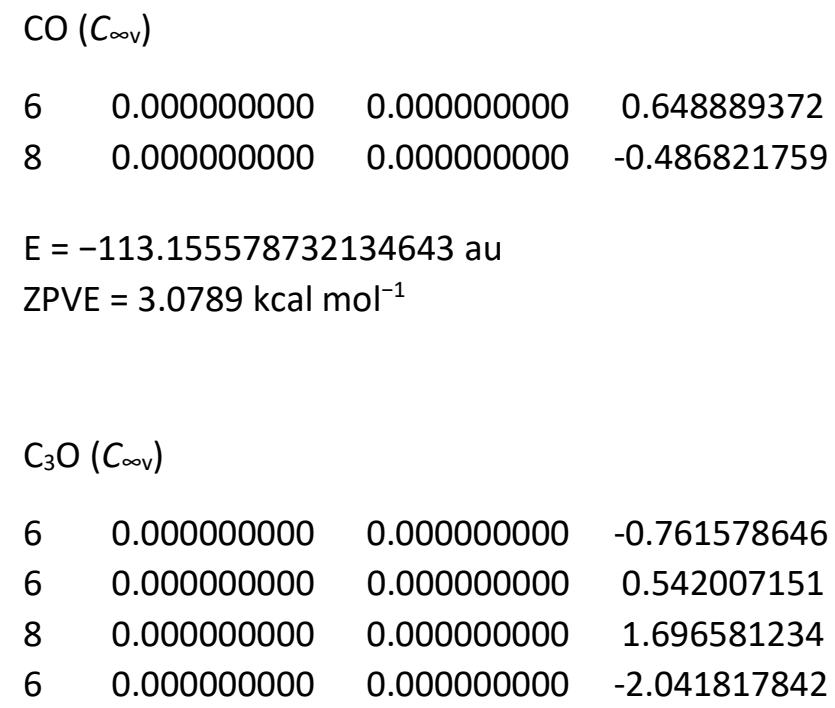




\begin{tabular}{lrrr}
\multicolumn{4}{c}{ Propadienone 4 $\left(C_{S}\right)$} \\
6 & 1.892836645 & 0.169937870 & 0.000000000 \\
6 & 0.662243144 & -0.352842589 & 0.000000000 \\
1 & 2.766863133 & -0.473275890 & 0.000000000 \\
1 & 2.078007120 & 1.240807035 & 0.000000000 \\
6 & -0.601580111 & 0.012981607 & 0.000000000 \\
8 & -1.770861448 & 0.079121413 & 0.000000000
\end{tabular}

$E=-190.340377578544746 \mathrm{au}$

$Z P V E=23.3665 \mathrm{kcal} \mathrm{mol}^{-1}$

Cyclopropenone $3\left(C_{2 v}\right)$

$\begin{array}{llll}8 & 0.000000000 & 0.000000000 & 1.483013626 \\ 6 & 0.000000000 & 0.000000000 & 0.278634629 \\ 6 & 0.000000000 & 0.676390296 & -0.991275074 \\ 1 & 0.000000000 & 1.552613182 & -1.624136096 \\ 6 & 0.000000000 & -0.676390296 & -0.991275074 \\ 1 & 0.000000000 & -1.552613182 & -1.624136096\end{array}$

$E=-190.326976728064693 \mathrm{au}$

ZPVE $=23.5642 \mathrm{kcal} \mathrm{mol}^{-1}$

$6\left(C_{s}\right)$

$\begin{array}{llll}6 & -2.070638264 & -0.095359788 & 0.000000000 \\ 6 & -0.775155747 & -0.016643402 & 0.000000000 \\ 6 & 0.469903550 & 0.360499889 & 0.000000000 \\ 8 & 1.635855605 & -0.215462042 & 0.000000000 \\ 1 & 2.327105076 & 0.460731273 & 0.000000000\end{array}$

$E=-189.596354699507941 \mathrm{au}$

$Z P V E=15.7324 \mathrm{kcal} \mathrm{mol}^{-1}$

$7\left(C_{1}\right)$

$\begin{array}{lrrr}6 & -0.896499530 & 0.500233148 & -0.166584813 \\ 6 & 0.558742334 & 0.673398036 & 0.178658521 \\ 6 & -1.390542119 & -0.715520958 & 0.100971226 \\ 1 & -1.492097832 & 1.437654976 & -0.094269551 \\ 8 & 1.253581019 & -0.420472701 & -0.087994197 \\ 1 & 2.175421180 & -0.219088044 & 0.144794664\end{array}$

$E=-190.189495822531910 \mathrm{au}$

ZPVE $=22.0069 \mathrm{kcal} \mathrm{mol}^{-1}$ 
$8\left(C_{s}\right)$

$\begin{array}{lrrr}6 & -1.573995197 & 0.563689374 & 0.000000000 \\ 6 & -0.822731142 & -0.603455764 & 0.000000000 \\ 1 & -0.931139393 & 1.460631825 & 0.000000000 \\ 6 & 0.500457461 & -0.248029721 & 0.000000000 \\ 1 & -1.172016733 & -1.625532807 & 0.000000000 \\ 8 & 1.555171786 & 0.226305974 & 0.000000000\end{array}$

$E=-190.289505163081600 \mathrm{au}$

ZPVE $=22.7976 \mathrm{kcal} \mathrm{mol}^{-1}$

Hydrogen atom

$E=-0.499809811301843 a u$

$\mathrm{H}_{2}\left(D_{\infty h}\right)$

$\begin{array}{llll}1 & 0.000000000 & 0.000000000 & 0.371311520\end{array}$

$\begin{array}{llll}1 & 0.000000000 & 0.000000000 & -0.371311520\end{array}$

$E=-1.172336686770175 \mathrm{au}$

ZPVE $=6.3035 \mathrm{kcal} \mathrm{mol}^{-1}$

$27\left(C_{s}\right)$

$\begin{array}{rrrr}6 & 1.591824634 & 0.022202642 & 0.000000000 \\ 6 & 0.284751966 & -0.182014949 & 0.000000000 \\ 1 & 2.252530449 & -0.832630921 & 0.000000000 \\ 1 & 2.008484147 & 1.019950676 & 0.000000000 \\ 6 & -1.062766265 & -0.699965904 & 0.000000000 \\ 8 & -0.879034469 & 0.633235827 & 0.000000000\end{array}$

$E=-190.258027794542102 \mathrm{au}$

ZPVE $=22.7601 \mathrm{kcal} \mathrm{mol}^{-1}$ 
$28\left(C_{s}\right)$

$\begin{array}{rrrr}6 & 0.131684155 & -0.004760230 & 0.000000000 \\ 6 & -1.042379272 & -0.628545449 & 0.000000000 \\ 8 & 1.449512462 & -0.082830591 & 0.000000000 \\ 6 & -1.033103742 & 0.802070855 & 0.000000000 \\ 1 & -1.651749883 & -1.516887653 & 0.000000000 \\ 1 & 1.791415205 & 0.822011207 & 0.000000000\end{array}$

$E=-190.277264143332275 \mathrm{au}$

ZPVE $=24.1009 \mathrm{kcal} \mathrm{mol}^{-1}$

TS7: $\mathbf{2 7} \rightarrow \mathbf{4}\left(C_{\mathrm{s}}\right)$

$\begin{array}{llll}6 & 1.725216978 & 0.202842466 & 0.000000000 \\ 6 & 0.513837200 & -0.232880842 & 0.000000000 \\ 1 & 2.546953648 & -0.503610892 & 0.000000000 \\ 1 & 1.936031891 & 1.264571332 & 0.000000000 \\ 6 & -0.809297469 & -0.685333873 & 0.000000000 \\ 8 & -1.355127302 & 0.488752346 & 0.000000000\end{array}$

$E=-190.201360175631635 \mathrm{au}$

ZPVE $=20.2688 \mathrm{kcal} \mathrm{mol}^{-1}$

$v_{\mathrm{i}}=1406.9619 i \mathrm{~cm}^{-1}$

TS8: $\mathbf{1 t} \rightarrow \mathbf{2 8}\left(C_{1}\right)$

$\begin{array}{llll}6 & 0.881609445 & 0.702977600 & -0.082108311\end{array}$

$\begin{array}{lllll}6 & 1.305004987 & -0.475079083 & 0.181012428\end{array}$

$\begin{array}{lllll}1 & 0.583596541 & 1.717688241 & -0.246164699\end{array}$

$\begin{array}{llll}6 & -0.287358645 & -0.577457381 & -0.251024139\end{array}$

$\begin{array}{llll}8 & -1.328811402 & 0.176061044 & 0.135129062\end{array}$

$\begin{array}{llll}1 & -2.108508564 & -0.349767244 & -0.087164436\end{array}$

$E=-190.175100514092094 \mathrm{au}$

ZPVE $=21.1471 \mathrm{kcal} \mathrm{mol}^{-1}$

$v_{\mathrm{i}}=596.4921 i \mathrm{~cm}^{-1}$ 
TS9: $1 \mathrm{c} \rightarrow \mathbf{4}\left(C_{s}\right)$

$\begin{array}{lrll}6 & 1.363637387 & 0.466288655 & 0.000000000 \\ 6 & 0.695622512 & -0.640562069 & 0.000000000 \\ 1 & 2.368141565 & 0.859032299 & 0.000000000 \\ 6 & -0.651673781 & -0.637503901 & 0.000000000 \\ 8 & -1.202589023 & 0.481903226 & 0.000000000 \\ 1 & -0.042067792 & 1.158507267 & 0.000000000 \\ & & \\ E & =-190.182509067527803 \mathrm{au} \\ \text { ZPVE }=19.5100 \mathrm{kcal} \mathrm{mol}^{-1} & \\ V_{\mathrm{i}}=1918.8503 \mathrm{~cm}^{-1} & \end{array}$




\section{References}

(1) Eckhardt, A. K.; Erb, F. R.; Schreiner, P. R. Conformer-specific [1,2]H-tunnelling in captodatively-stabilized cyanohydroxycarbene (NC- ̈-OH). Chem. Sci. 2019, 10, 802-808.

(2) Rossum, G. Van; Drake, F. L. Python 3 Reference Manual. 2009.

(3) Jones, E.; Oliphant, T.; Peterson, P. SciPy: Open source scientific tools for Python. 2001.

(4) Hunter, J. D. Matplotlib: A 2D graphics environment. Comput. Sci. Eng. 2007, 9, 90-95.

(5) Harris, C. R.; Millman, K. J.; van der Walt, S. J.; Gommers, R.; Virtanen, P.; Cournapeau, D.; Wiesner, E.; Taylor, J.; Berg, S.; Smith, N. J.; Kern, R.; Picus, M.; Hoyer, S.; van Kerkwijk, M. H.; Brett, M.; Haldane, A.; Fernández del Río, J.; Wiebe, M.; Peterson, P.; Gérard-Marchant, P.; Sheppard, K.; Reddy, T.; Weckesser, W.; Abbasi, H.; Gohlke, C.; Oliphant, T. E. Array programming with NumPy. Nature 2020, 585, 357-362.

(6) Becke, A. D. Correlation energy of an inhomogeneous electron gas: A coordinate-space model. J. Chem. Phys. 1988, 88, 1053-1062.

(7) Lee, C.; Yang, W.; Parr, R. G. Development of the Colle-Salvetti correlation-energy formula into a functional of the electron density. Phys. Rev. B 1988, 37, 785-789.

(8) Weigend, F.; Ahlrichs, R. Balanced basis sets of split valence, triple zeta valence and quadruple zeta valence quality for $\mathrm{H}$ to $\mathrm{Rn}$ : Design and assessment of accuracy. Phys. Chem. Chem. Phys. 2005, 7, 3297-3305.

(9) Weigend, F. Accurate Coulomb-fitting basis sets for H to Rn. Phys. Chem. Chem. Phys. 2006, 8, 1057-1065.

(10) Purvis, G. D.; Bartlett, R. J. A full coupled-cluster singles and doubles model: The inclusion of disconnected triples. J. Chem. Phys. 1982, 76, 1910-1918.

(11) Raghavachari, K.; Trucks, G. W.; Pople, J. A.; Head-Gordon, M. A fifth-order perturbation comparison of electron correlation theories. Chem. Phys. Lett. 1989, 157, 479-483.

(12) Dunning, T. H. Gaussian basis sets for use in correlated molecular calculations. I. The atoms boron through neon and hydrogen. J. Chem. Phys. 1989, 90, 1007-1023.

(13) Peterson, K. A.; Woon, D. E.; Dunning, T. H. Benchmark calculations with correlated molecular wave functions. IV. The classical barrier height of the $\mathrm{H}^{+} \mathrm{H}_{2} \rightarrow \mathrm{H}_{2}+\mathrm{H}$ reaction. J. Chem. Phys. 1994, 100, 7410-7415.

(14) Woon, D. E.; Dunning, T. H. Gaussian basis sets for use in correlated molecular calculations. V. Core-valence basis sets for boron through neon. J. Chem. Phys. 1995, 103, 4572-4585.

(15) Kendall, R. A.; Dunning, T. H.; Harrison, R. J. Electron affinities of the first-row atoms revisited. Systematic basis sets and wave functions. J. Chem. Phys. 1992, 96, 6796-6806.

(16) Glendening, E. D.; Landis, C. R.; Weinhold, F. Natural bond orbital methods. Wiley Interdiscip. Rev. Comput. Mol. Sci. 2012, 2, 1-42.

(17) Ekern, S.; Szczepanski, J.; Vala, M. An ab Initio Study of the $\mathrm{C}_{3} \mathrm{H}_{2} \mathrm{O}$ Potential Surface: A Mechanism for Propynal Formation and Destruction. J. Phys. Chem. 1996, 100, 16109-16115.

(18) Ekern, S.; Vala, M. Theoretical Study of Photochemical Mechanisms of $\mathrm{C}_{3} \mathrm{O}$ Formation. J. Phys. Chem. A 1997, 101, 3601-3606.

(19) Szczepanski, J.; Ekern, S.; Vala, M. Spectroscopy and Photochemistry of the $\mathrm{C}_{3} \cdot \mathrm{H}_{2} \mathrm{O}$ Complex in Argon Matrices. J. Phys. Chem. 1995, 99, 8002-8012. 
(20) Mardyukov, A.; Quanz, H.; Schreiner, P. R. Conformer-specific hydrogen atom tunnelling in trifluoromethylhydroxycarbene. Nat. Chem. 2016, 9, 71-76. 


\section{Full Citations for Electronic Structure Codes}

Gaussian 16

M. J. Frisch, G. W. Trucks, H. B. Schlegel, G. E. Scuseria, M. A. Robb, J. R. Cheeseman, G. Scalmani, V. Barone, G. A. Petersson, H. Nakatsuji, X. Li, M. Caricato, A. V. Marenich, J. Bloino, B. G. Janesko, R. Gomperts, B. Mennucci, H. P. Hratchian, J. V. Ortiz, A. F. Izmaylov, J. L. Sonnenberg, D. Williams-Young, F. Ding, F. Lipparini, F. Egidi, J. Goings, B. Peng, A. Petrone, T. Henderson, D. Ranasinghe, V. G. Zakrzewski, J. Gao, N. Rega, G. Zheng, W. Liang, M. Hada, M. Ehara, K. Toyota, R. Fukuda, J. Hasegawa, M. Ishida, T. Nakajima, Y. Honda, O. Kitao, H. Nakai, T. Vreven, K. Throssell, J. A. Montgomery, Jr., J. E. Peralta, F. Ogliaro, M. J. Bearpark, J. J. Heyd, E. N. Brothers, K. N. Kudin, V. N. Staroverov, T. A. Keith, R. Kobayashi, J. Normand, K. Raghavachari, A. P. Rendell, J. C. Burant, S. S. Iyengar, J. Tomasi, M. Cossi, J. M. Millam, M. Klene, C. Adamo, R. Cammi, J. W. Ochterski, R. L. Martin, K. Morokuma, O. Farkas, J. B. Foresman, D. J. Fox, Gaussian 16 Revision C.01, 2016, Gaussian Inc., Wallingford.

\section{CFOUR}

CFOUR, a quantum chemical program package written by J.F. Stanton, J. Gauss, L. Cheng, M. E. Harding, D. A. Matthews, P. G. Szalay with contributions from A. A. Auer, R. J. Bartlett, U. Benedikt, C. Berger, D. E. Bernholdt, Y. J. Bomble, O. Christiansen, F. Engel, R. Faber, M. Heckert, O. Heun, M. Hilgenberg, C. Huber, T.-C. Jagau, D. Jonsson, J. Jusélius, T. Kirsch, K. Klein, W. J. Lauderdale, F. Lipparini, T. Metzroth, L. A. Mück, D. P. O'Neill, D. R. Price, E. Prochnow, C. Puzzarini, K. Ruud, F. Schiffmann, W. Schwalbach, C. Simmons, S. Stopkowicz, A. Tajti, J. Vázquez, F. Wang, J. D. Watts and the integral packages MOLECULE (J. Almlöf and P. R. Taylor), PROPS (P. R. Taylor), ABACUS (T. Helgaker, H. J. Aa. Jensen, P. Jørgensen, and J. Olsen), and ECP routines by A. V. Mitin and C. van Wüllen. For the current version, see http://www.cfour.de.

\section{Polyrate}

J. Zheng, J. L. Bao, R. Meana-Pañeda, S. Zhang, B. J. Lynch, J. C. Corchado, Y.-Y. Chuang, P. L. Fast, W.P. Hu, Y.-P. Liu, G. C. Lynch, K. A. Nguyen, C. F. Jackels, A. Fernandez Ramos, B. A. Ellingson, V. S. Melissas, J. Villà, I. Rossi, E. L. Coitiño, J. Pu, T. V. Albu, A. Ratkiewicz, R. Steckler, B. C. Garrett, A. D. Isaacson, D. G. Truhlar, Polyrate Version 2017-C, 2017, University of Minnesota, Minneapolis.

\section{NBO7}

E. D. Glendening, J, K. Badenhoop, A. E. Reed, J. E. Carpenter, J. A. Bohmann, C. M. Morales, P. Karafiloglou, C. R. Landis, F. Weinhold, NBO 7.0, 2018, Theoretical Chemistry Institute, University of Wisconsin, Madison. 